$$
1961
$$

$$
6 /-39
$$

volumie I

$$
\text { copy } 1
$$

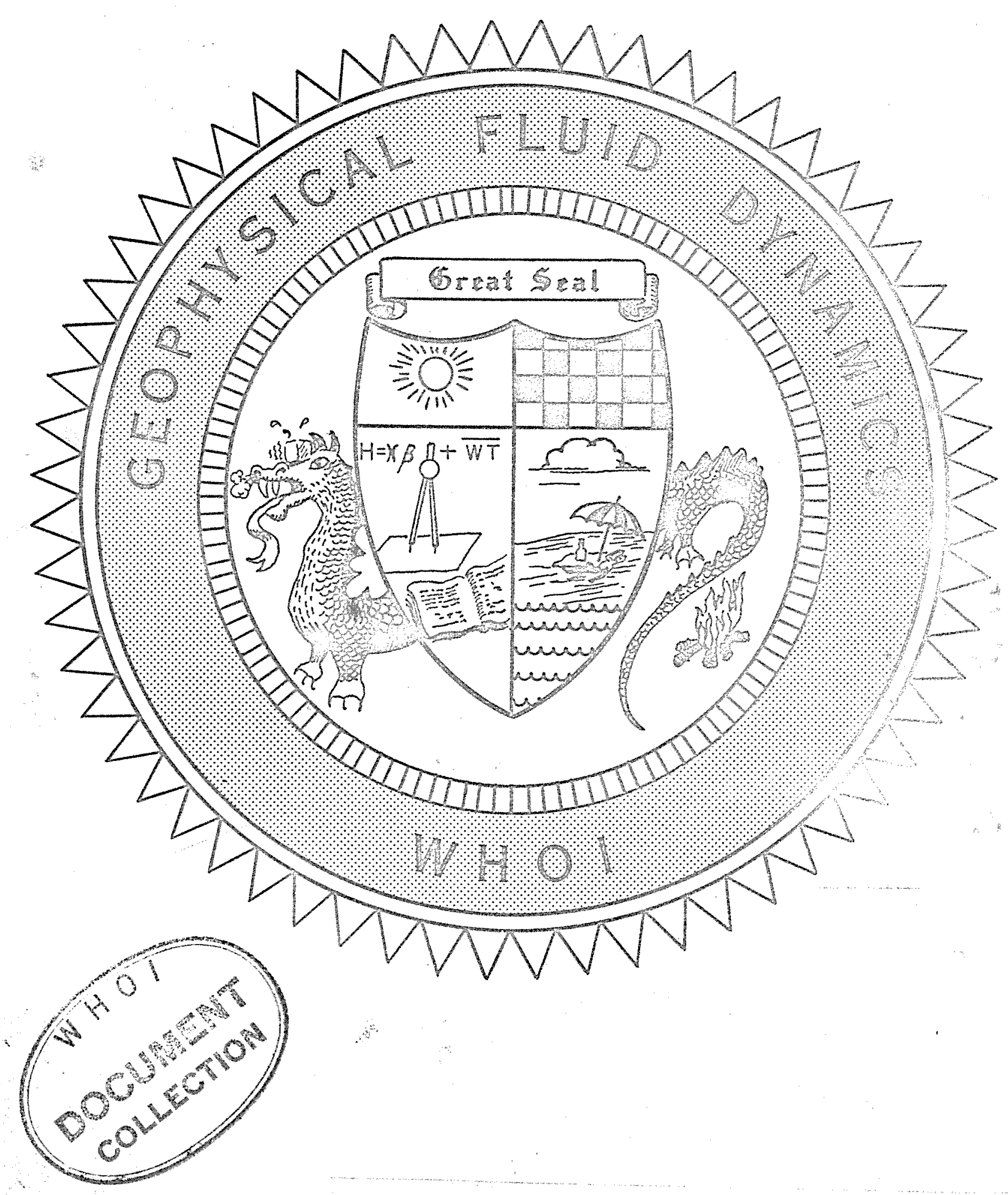

Veronis Lectures 


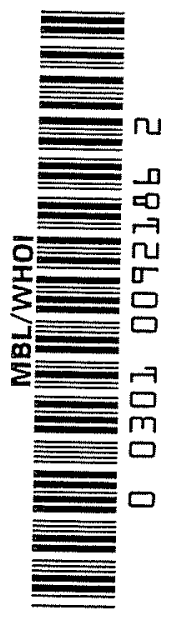




\section{Goncents of the Wolumes}

\section{Volume I. Student Noces of lectures by George Veronds}

on Geophysical wud Dynames.

Volume II. Lectures by N.P. Pofonofi

on Energy Transformations in the ocma.

Volugne MI. Participants' Lectures. 


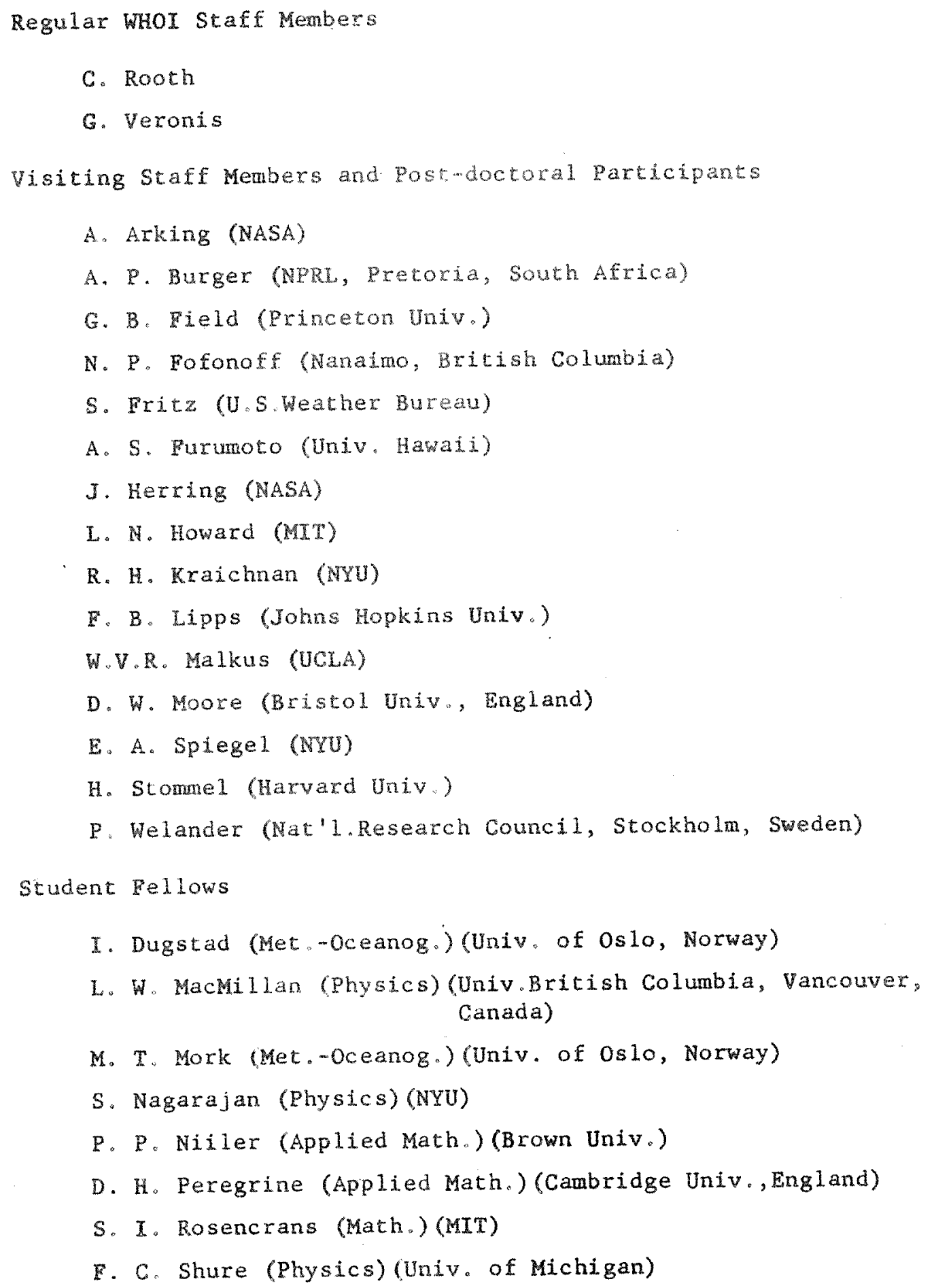




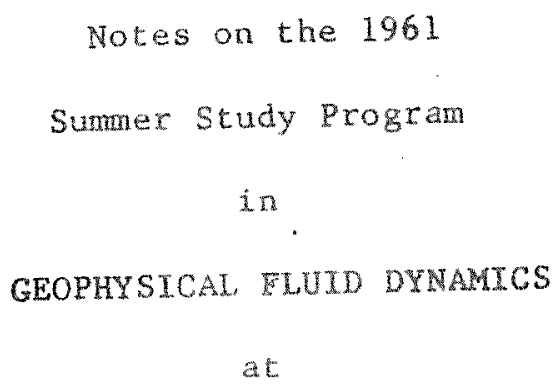


One expects an Oceanographic Institution to encourage the study of the motions of the sea. However, an explanation is needed for the Woods Hole tradition of teaching and research across the entire spectrum of geophysical fluid dynamics. The motions in the earth's core and in its liquid and gaseous envelopes, the motions in the sun and in its tenuous atmosphere; all are flows caused by the convection of heat and the transfer of momentum in rotating systems. Understanding in one area is usually a contribution to understanding in a11. By encouraging study with a broad base the theoretical group at Woods Hole has been able to gain from the enthusiasms and skills of meteorologists, physicists, mathematicians and astrophysicists.

This report of the third summer course in Geophysical Fluid Dynamics attests to the new tools and ideas, drawn from many disciplines, which now enrich oceanographic study.

The success of this third course is due primarily to the efforts of Dr. George Veronis. He has acted both as Director of the study program and as the principal staff lecturer.

The first volume of these notes is a restatement by the students of Dr. Veronis' introductory lectures. Despite the considerable differences in presentation, the notes preserve the content and spirit of the lecture material.

The editor and students wish to thank Mrs. Mary Thayer for her capable assistance in assembling and reproducing these notes. We are all indebted to the National Science Foundation for its continuing support of the Summer Study Program.

Willem V.R. Malkus 


\section{Lecturex's Foreward}

Any attempt to lecture on a field as broad as theoretical geophysical fluid dynamics must reflect the lecturer's philosophy. My own view is that the study of geophysical hydrodynamics is composed of a combination of three types of investagation. In decreas ing order of rigor they consist of theoretical investigations into: a) fluid processes of a general nature; b) laboratory experiments modelled on a specific geophysical phenomenon; and c) theoretical models of actual geophysical phenomene

In the limited time allowed for these lectures I chose to treat an example of the two extremes. As an example of class a) thermal convection is treated in a quite rigorous fashion with the analysis proceeding from the pure conductive state through the stability problen to finite amplitude motions and finally to fullydeveloped turbulence. tr class c) the steady, wind-driven, ocean circulation is the area of investigation. The artempt has been to study the evolution of the theory from the earlier linear models to the latest models which are non linear.

It was not intended that the students notes be a simple record of the lectures. In some cases the material was reworked by the student and the presentation reflects much of the student's own orientation and interest. In other cases, the notes are much closer to the presentation in the lecture room. In all cases, the outline of the lectures has been adhered to and the material is essentially that covered in the lectures. 


\section{Veronis Lectures}

Table of Contents

PART I Equations of Mation

Introduction

I. Particle Mechanics

II. The Gibbsian Ensemble

III. Probability Function for a Single Molecule 3

IV. Thermodynamic Variables 4

V. Introduce Specific Macroscopic Assumptions 5

VI. The Navier-Stokes Equations for a Monatomic Gas 5

PART II Thermal Convection

I. Experimental Results for Simple Convection 7

1. Description of the Experiment 7

2. Experimental Values for $R_{c}$

3. Cellular Motion 9

4. The End of the Laminar Convectivd Range 10

References 12

II. The Equations describing Simple Convection 13

1. Boussinesq's Approximation 13

Derivation of Boussinesq's Equations $\quad 14$

Navier-stokes equations 17

References 19

III. Stability Theory 20

Digression on Fourier series 25

A Variational Method for Computing $\mathrm{R}_{\mathrm{c}} \quad 29$

IV. Cells 31

V. Finite Amplitude (non-1inear) Theory 33

Introduction 33

The Finite Amplitude Expansion 38

VI. A Relative Stability Criterion 44

Relative Stability and Entropy Production 48

VII. Turbulent Convection 50

Free Boundary Condition Solutions $\quad 52$

Rigid Boundary Condition Solutions $\quad 57$ 
Table of Contents (2) Page

Critical Remarks and Comparisons wth other

Theories 58

Comparison with Experiments 59

References 61

PART III The Wind-driven Ocean Circulation

I. The Observational Basis 63

1. Introduction 63

2. Geostrophic Currents Associated with Mean Density Field 64

General References 69

II. Equations for Oceanic Motions 70

1. The $\beta$ mplane Approximation 70

2. A Word on Friction 79

III. Ekman's Theory of Wind-drift 79

1. Steady Flow 79

2. Digression on Inertial Flow 84

3. The Time-dependent Ekman Wind-drift 85

References $\quad 89$

IV. Geostrophic Motion 89

V, Sverdrup's Theory of Wind-generated Transport 90

Reference 93

VI. Stomel's Theory of Western Intensification 94

Reference 99

VII. Munk's Theory of the Wind-driven Ocean
Circulation

1. The Assumptions and Formulations 100

2. Munk's Solution 102

3. The Representations of Results 107

VIII. Ocean Circulation - Two-layer Model 109

1. The Ocean Density Field 109

2. One-layer Mode1 of Constant Depth
(Fofonoff Inviscid System)

3. Case of Constant East-West Flow in the Basin 114 
IX. The Inertial Theories of Stomel, charney and Morgan

1. Basic Considerations and Fonulations

2. Stommel's Model

3. Morgan's Model of the Homogeneous Ocean

4. The Solution According to Charney

X. General Discussion of the ocean Circulation 126

1. In Recrospect 126

2. The Single-1ayer System 127

3. The Two-layer Systen 129

4. Carrier and Robinson on The MidLatitude Jet

XI. Summary 


LECTURES by George Veronis Summer, 1961

\section{Introduction}

For the purposes of geophysical fluid dynamics the derivation of the Navier-Stokes equations by means of continum mechanics is, perhaps, not the most appropiate derivation. In this lecture we will indicate a gas-dynamical derivation in four steps, after each of which the resulting equations describe less closely, but with less irrelevant information, the motion of the system.

I. Particle Mechanics: (based on classical mechantcs since we will be concerned with macroscoplc properties.)

From this first point of view, the system, a group of particles, has its state specifled by $P$, a point in phase space, where

$$
\mathrm{P}=\left(\mathrm{q}_{j}^{\mathrm{i}}, \mathrm{P}_{j}^{\mathrm{i}}\right) i=1,2, \ldots \mathrm{n} ; j=1,2, \ldots \mathrm{s} \text {. }
$$

$q_{j}^{i}$ are the postion comordinates of a particle, and $p_{j}^{i}$ are its momenta; the number of degrees of freedom of a particle is $s$, and the number of particles in the system is $n$.

If $P$ is given at an initial time, the subsequent state of the system is determined by solving Hamilton's equations. Any physical quantity $\phi$ would be a function of $P, \phi(P)$. Serious objections to this procedure are

a) It provides more information than we want, or could possibly use, for the solution would be given by particle trajectories. 
b) it is at present impossible to solve the problem for more than two particles.

Remark: From the particle point of view, solving for the motion in a bathtub in which the water is at rest, is as difficult a problem as solving for the notion when the water has been disturbed so that the resultant motion is turbulent.

\section{The Gibbsian Ensemble.}

Consider a large number of replicas of the system and a function $(W(P, t)$ representing the proportion of replicas in state $p$ at time $t$. In the limit of an infinite number of systems, $W$ becomes a continuous function representing the probability that the system is in state P. W satisfies the Liouville equation and can be calculated for all $t$, if it is known at an initial instant. The physical quantity (P) may now be replaced by its expected value,

$$
\bar{\phi}=\int W(P, t) \phi(P) d P
$$

Note that in the transition from I to II the description has changed from $P$ to $W$ and certainty has been replaced by probability. An advantage of this method is that we are now able to discard improbable states. Objections to the Gibbsian approach are that the amount of information is still too great, and that the problem is still in practice insoluble. 
III. Probability Function for a Single Molecule.

We defind

$$
w_{1}\left(p_{j}^{1}, q_{j}^{1}\right)=\int w d p^{\prime}
$$

where

$$
d p^{\prime}=\prod_{\substack{i{ }^{\prime} \\ a 11}} d p_{j}^{i} d q_{j}^{i} .
$$

$\mathrm{dP}^{\prime}$ is a volume element of phase space including all dimensions $p_{j}^{i}, q_{j}^{i}$ except those corresponding to a single molecule. $W$, is then, clearly, the probability function for a single molecule. As before, for a physical quantity $\phi$ we define

$$
\bar{\phi}=\int w_{1}\left(P_{1}\right) \phi\left(P_{1}\right) d P_{1} \text {, }
$$

where

$$
p_{1}=\left(p_{j}^{1}, q_{j}^{1}\right)
$$

If we define a distribution function

$$
F\left(P_{1}\right)=n W_{1}\left(P_{1}\right)
$$

it has the property that $F_{1} P_{1}$ is the number of molecules in an element $d p_{1}$ of phase space.

Now restrict attention to a monatomic gas. The distribution function becomes $F(\underline{r}, \underline{v}, t)$; $\underline{r}$ is the position coordinate and $\underline{v}$ the velocity. Consider gas nolecules in the vicinity of ( $\underline{\underline{v}} \underline{\mathrm{v}}, t)$, after time $\Delta t$ they are at $(\underline{\underline{r}}+\Delta \underline{\underline{x}}, \underline{\mathbf{v}}+\Delta \underline{\mathrm{v}}, t+\Delta t)$. The difference, if any, of the number of particles at these two points is $F(\underline{r}+\Delta \underline{\underline{r}}, \underline{v}+\Delta \underline{v}, t+\Delta t)-F(\underline{r}, \underline{v}, t)$. 
We write

$$
F(\underline{r}+\Delta \underline{r}, \underline{y}+\Delta \underline{v}, t+\Delta t)-F(\underline{r}, \underline{v}, t)=\Delta t\left(\frac{d F}{d t}\right) \operatorname{coll}
$$

thus indicating that any change in $F$ is due to collisions. In the limit as $\Delta t \rightarrow 0$,

$$
\frac{\partial F}{\partial t}+\left(\underline{v} \cdot \nabla_{\underline{\underline{F}}}\right) F+\left(\underline{a} \cdot \nabla_{\underline{v}}\right) F=\left(\frac{d F}{d t}\right) \operatorname{coll} .
$$

This is the Boltzmann Equation. The term $\left(\frac{d F}{d t}\right)_{c o l l}$ is unknown but various expressions may be used by assuning special mechanisms of collision. This is the domain of kinetic theory. For a given macroscopic state there may be several joint probability functions $w$. In the transition from II to III several such functions may be made to correspond to the sane one-molecule distribution function $F$. Thus we may have eliminated some surplus information.

IV. Thermodynamic Variables.

At this stage a larger step is taken. Conservation equations for the macroscopic quantities mass, momentum and energy can be derived from the Boltzmann equation (in which the function $\left(\frac{d E}{d t}\right)_{c o l 1}$ is assumed known). This system of equations is closed by glving specific forms of the stress tensor and of the heat vector. After the transition from III to IV we have finally lost much of the "non-useful" information which was present in the earlier formulations. Possibly a number of distributions $F$ are now equivalent to the same macroscopic state. 


\section{Introduce Specific Macroscopic Assumptions.}

The complete equations are still difficult to solve.

We may go one step further and restrict our attention to particular, hypothetical, cases, e.g. incompressible, inviscid flow.

\section{The Navier-Stokes Equations for a Monatomic Gas.}

The conservation equations mentioned above yield the Navier-Stokes equations, which apply to systems near the equilibrium state. These equations are not universally applicable. Far from the equilibrium state their use has to be carefully considered, such as in strong shock waves, where changes occur in a distance comparable to the mean free path of a molecule.

The equations for conservation of momentum are

$$
\frac{\partial u_{i}}{\partial t}+u_{j} u_{i, j}=\sigma_{i j, j}+f_{i} .
$$

$f_{1}$ is the body force, and $\sigma_{i j}$ the stress tensor,

$$
\sigma_{i j}=-p \delta_{i j}+\mu\left(u_{i, j}+u_{j, i}\right)+\lambda u_{k, k} \delta_{i j} .
$$

The formula for $\sigma_{i f}$ is based on an additional assumption of local isotropy. $\mu$ is the first coefficient of viscosity; $\lambda$ is the second. They are functions of the thermodynamic variables. A further restriction to the case, $\mu=$ constant, $\lambda=-\frac{2}{3} \mu$, which is valid when $\lambda$ is independent of the rate of compression, gives the more familiar form

$$
\frac{\mathrm{Du}}{\mathrm{Dt}}=-\frac{1}{\rho} \nabla \mathrm{P}+\gamma \nabla^{2} \underline{\mathrm{v}}+\frac{\gamma}{3} \nabla(\nabla \cdot \underline{\mathrm{v}})+\underline{\mathrm{f}},
$$

where $\gamma=\mu / \rho$. 
The equation for the conservation of mass is

$$
\frac{\partial \rho}{\partial t}+\nabla \cdot(\rho \underline{v})=0
$$

The equation for the conservation of energy is

$$
\rho \mathrm{C}_{\mathrm{v}} \frac{\mathrm{DT}}{\mathrm{DC}}+\mathrm{P} \nabla \cdot \underline{\mathrm{v}}=K \nabla^{2} \mathrm{~T}+\mathrm{Q}+\phi .
$$

$\mathrm{C}_{\mathrm{v}}$ is the specific heat at constant volume. Q represents

internal heat sources. $\phi$ is the dissipation term, and with the assumption (2),

$$
\phi=\lambda(\nabla \cdot \underline{v})^{2}+\frac{1}{2} \mu\left(u_{i, j}+u_{j, i}\right)^{2} .
$$

With the equation of state

$$
\rho=P(P, T)
$$

(3), (4) and (5) form a closed set of equations.

In addition, a property $S$, which is identified with the entropy of the system, is defined by

$$
\rho T \frac{d S}{d t}=K \nabla^{2} T+Q+\phi .
$$




\section{Convection}

\section{Experimental Results for Simple Convection.}

1. Description of the experiment.

Consider a layer of fluid of depth d between two horim zontal, perfectly conducting planes. The upper and lower planes are at temperatures $\mathrm{T}_{\mathrm{C}}$ and $\mathrm{T}_{\mathrm{H}}$ respectively, where $\mathrm{T}_{\mathrm{H}}>\mathrm{T}_{\mathrm{C}}$ and both are kept constant. The fluid has coefficient of thermal expansion $\alpha$, kinematic viscosity $\gamma$ and themal conductivity $k$. The temperature difference produces a gravitational instability, for it induces a density stratification in which density is greatest at the upper plane. However, in this problem, viscosity is a stabilizing influence, and the system is stable to infinitesimal disturbances as long as the potential energy due to the temperature difference and the gravitational field is less than a certain "threshold" energy necessary to maintain the field of motion against dissipative forces.

Mathematical analysis shows that the system is stable for $R<R_{C}$ where $R$ denotes the Rayleigh number,

$$
R=\frac{g \alpha \Delta T d^{3}}{x \gamma}=\frac{g \alpha \frac{\Delta T}{d}}{\frac{x}{d^{2}} \frac{\gamma}{d^{2}}},
$$

where $\Delta \mathrm{T}=\mathrm{T}_{\mathrm{H}}-\mathrm{T}_{\mathrm{C}}$. The second form of $\mathrm{R}$ displays it as a ratio of the potential energy to the dissipation and heat conduction. The valut of $R_{C}$ depends on the boundary conditions at the two planes. These boundary conditions correspond to either a rigid or a free surface. If there is a rigid surface then we require $\underline{v}=0$ on it. If the surface is free than $\underline{v} \cdot \underline{\underline{u}}=0$ where $\underline{\underline{a}}$ is the 
normal to the surface, and continuity of the stress-tensor across the boundary is required.

In the "rigid-free" case (lower rigid, upper free)

$\mathrm{R}_{\mathrm{C}} \simeq 1100$. In the rigid-rigid case, $\mathrm{R}_{\mathrm{C}}=1708$. These are theoretical results.

\section{Experimental Values for $R_{C}$.}

In the experiments to be described rigid planes axe used. In all of them $\mathrm{R}_{\mathrm{C}}$ was deternined from the curve of $\mathrm{R} v \mathrm{v}$. Nu, the Nusselt number, given by

$$
N u=\frac{H_{/ \rho} c_{p}}{k\left(\frac{\Delta T}{d}\right)_{C}}
$$

where $\mathcal{H}$ is the total heat transport. Thus $\mathrm{Nu}$ is a measure of the ratio of total heat transport to conductive heat transport. It is found that the curve has the following form:

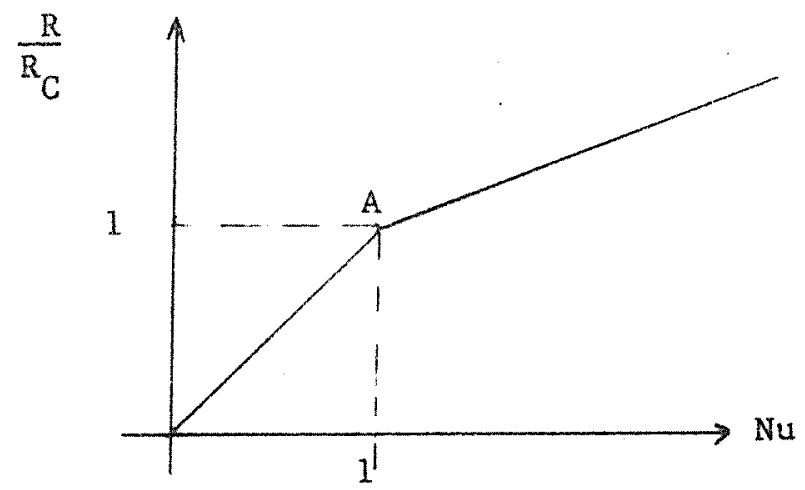

This shows that heat is transported by conduction for $R<R_{C}$. At $R=R_{C}$ convection begins and the heat flux increases sharply. Thus one can determine $R_{C}$ experimentally by plotting the above curve and locating point $A$. 
a. Schmidt and Milverton (1935) found $R_{C}=1770 \pm 140$, using water which was heated from below but not cooled from above - how ever, a constant check was kept on $\Delta \mathrm{T}$ and $\mathrm{it}$ was always $<2^{\circ} \mathrm{C}$. A depth of 4 to $5 \mathrm{~mm}$ was used, and 4 experiments wexe performed, with values of $R_{C}$ between 1580 and 1970.

b. Chandra (1938) had $R_{C}=1700$. The fluid was air, d was varied over a large range and $\triangle \mathrm{T}$ was up to $100^{\circ} \mathrm{C}$. (The Boussinesq approximation, which was used to derive the theoretical results is a poor approximation for such a large $\Delta \mathrm{T}$.)

c. Schmidt and Saunders (1938) obtained $\mathrm{R}_{\mathrm{C}}=1750$. They varied the depth between 4 and 5 ma, the mean temperature was kept constant and $\triangle \mathrm{T}$ was always less than $2^{\circ} \mathrm{C}$.

d. Malkus (1954) observed $\mathrm{R}_{\mathrm{C}}=1700 \pm 80$. Water and acetone were used in many experiments with a depth of $4.4 \mathrm{~mm}$ and $(\Delta \mathrm{T})_{\mathrm{C}}<$ $2^{\circ} \mathrm{C}$. This experiment was different from the previous ones. Initially a large temperature difference was applied and was then allowed to decay until motion stopped. The decay time was long compared to the time scale of the motion.

e. Silveston (1958) found $\mathrm{R}_{\mathrm{C}}=1700 \pm 51$. He used water, heptane, glycol and silicone oils. In each experiment the mean temperature was constant. The depth of liquid was between $1.35 \mathrm{~mm}$ and 14 min with $3^{\circ} \mathrm{C} \geqslant \Delta \mathrm{T} \geqslant 2^{\circ} \mathrm{C}$.

\section{Cellular Motion.}

We are now concerned with the state of motion for $R \geq R_{C}$. 
Convection is usually observed to occur in a cellular pattern when $R$ is not much greater than $R_{C}$. This was first observed by Bénard with "rigld-free" boundary conditions. Due to recent work of Pearson it is now believed that instability in the "rigid-free" case of Bénard's experiments was due to the variation of surface tension with temperature and not because of the reasons formerly supposed.

The following experimental results give the slope of the $R$ - Nu curve for $R_{C}<R<2 R_{C}$.
a. Schmidt and Milverton. $\quad 1.74<$ slope $<2.27$.

\subsection{2 was the mean of 4 experiments.}

b. Schnidt and Saunders. $\quad 1.55<$ slope $<2.89$, mean 1.9 . c. Malkus claimed $10 \%$ accuracy for 2.5 but his published work gives 2.9 in one graph and 2.5 in another. d. Silveston mean: $1.9-2.0$.

4. The End of the Laminar Convective Range.

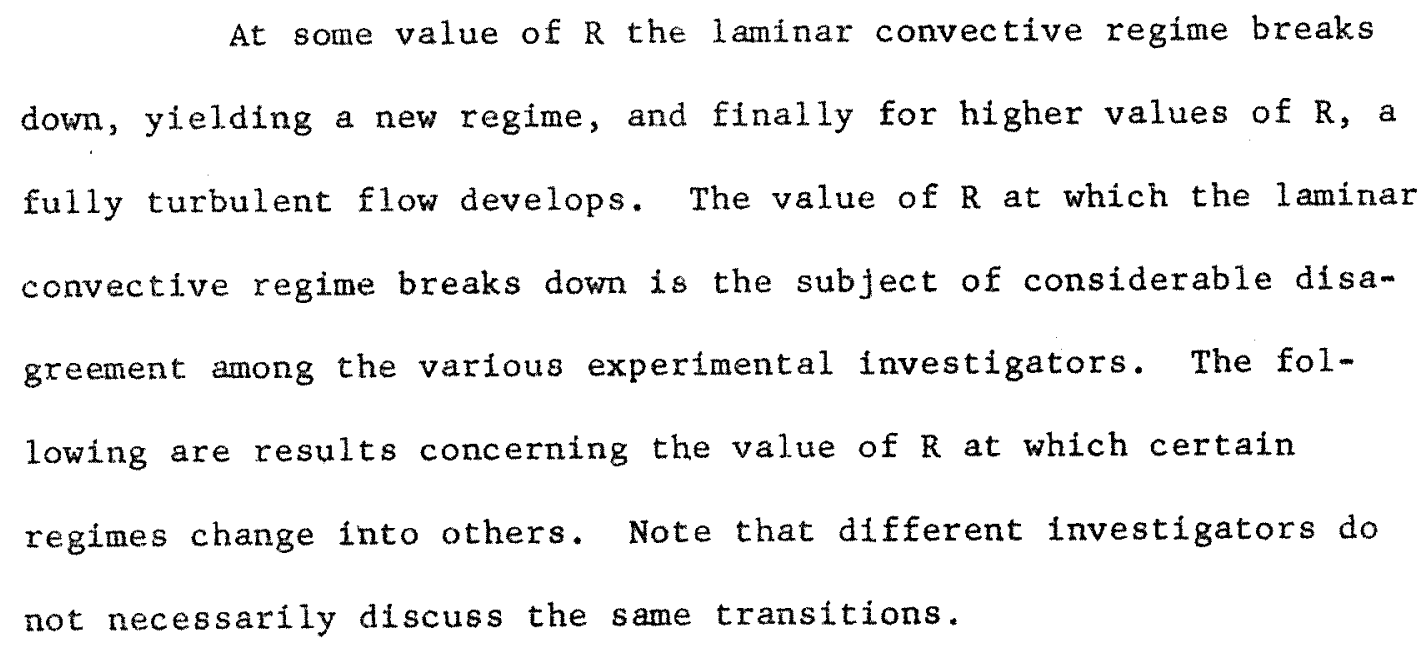


a. Mull and Reiher found the laminar regime in air broke down for an $\mathrm{R}<10,000$. For $10^{4}<\mathrm{Gr}<4.10^{5}$ they found $\mathrm{Nu}=.177 \mathrm{Gr}^{\frac{3}{4}}$ and for $\mathrm{Gr}>4.10^{5}, \mathrm{Nu}=.061 \mathrm{Gr}^{1 / 3}$. Gr is the Grashof number = $=\frac{\text { Rayleigh Number }}{\text { Prandt } 1 \text { Number }}$

For air the Prandtl Number $=0.7$.

b. Schnidt and Saunders found that the transition to the fully turbulent regime took place at $\mathrm{R} \simeq 44,000$ in water. For air they found transtion to a transient regime at $R \simeq 5000$, and transition to the turbulent regime at $R \simeq 44,000$

c. Silveston found a change in the slope of the R-Nu curve at $R \simeq 4,000$.

d. Malkus found the first change at $\mathrm{R} \simeq 17,000$. He believed he could detect transitions at six or seven places. His R-Nu curve could be well represented by a polygonal curve as shown.

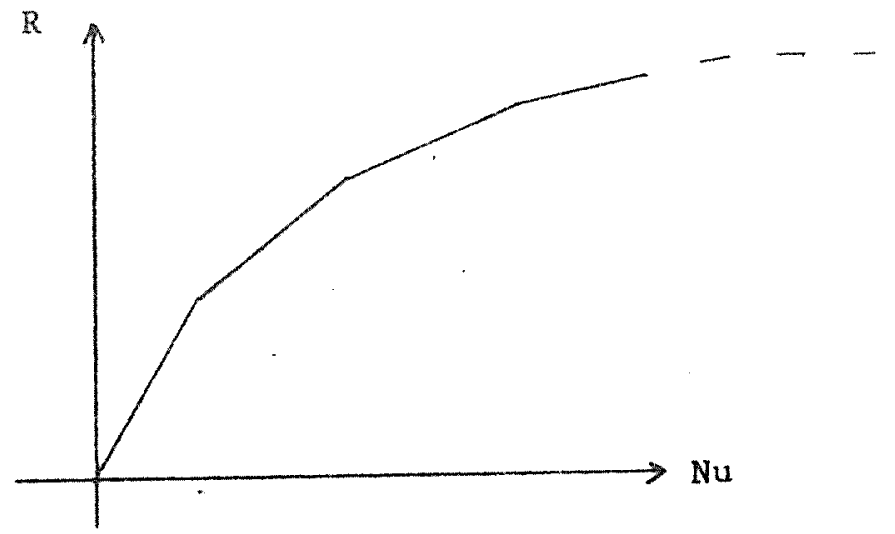

Very surprisingly the points of transition occur at values of $R$ corresponding to the critical $R$ at the higher modes in the linear stability problem. That is, if $R_{C}$ correspond to $n=1$, the rest 
correspond to $\mathrm{n}=2,3 \ldots$ The series of transition polnts he observed were 17,$000 ; 55,000 ; 170,000 ; 410,000 ; 850,000$ and 1,600,000. The odd ones were sharper than the even ones. Malkus also states that the $R$ vs. Nu curve can be described by power laws $\mathrm{Nu}=.198 \mathrm{R}^{\frac{3}{4}}$ and $\mathrm{Nu}=.079 \mathrm{R}^{1 / 3}$, but that the polygonal curve is more correct.

\section{References}

1. Chandra, 1938, Proc. Roy. Soc. A. 164.

2. Malkus, 1954, Proc. Roy, Soc. A. 215.

3. Pearson, 1958, J. Fluld Mech. 4, p.489.

4. Schmidt and Milverton, 1935, Proc. Roy. Soc, A. 152.

5. Schmidt and Saunders, 1938, Proc. Roy. Soc. A. 164.

6. Silveston, 1958, Forsch. Ing-Ves, 24 . 
II. The Equations Describing Simple Convection.

We shall consider the following idealization of the experimental problem. Two horizontal, perfectly conducting planes are a small distance $L$ apart. The top plane is maintained at a temperature $\Delta T$ below the temperature of the bottom plane

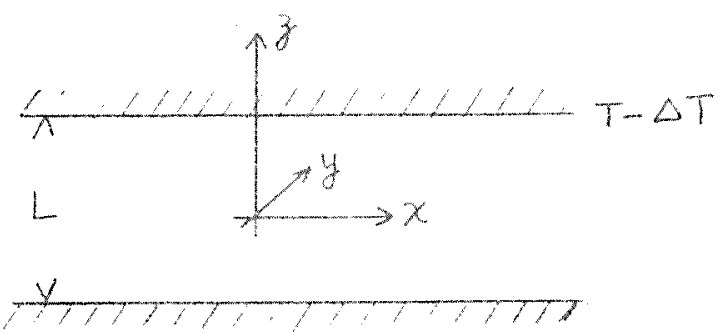

The first step is to simplify the Navier-stokes equations.

\section{Boussinesq's Approximation}

The main effect of this approximation is to neglect all density variations except those due to heat. The Boussinesq Equations, which will be derived, are

$$
\begin{gathered}
\frac{D \underline{v}}{D t}=-\frac{1}{\rho_{m}} \nabla P+g \propto\left(T-T_{m}\right) \underline{k}+\gamma \nabla^{2} \underline{v}, \\
\nabla \cdot \underline{v}=0,
\end{gathered}
$$

and

$$
\frac{D T}{D t}+\frac{g W}{C_{P}}=\frac{k}{\rho_{m} C_{P}} \nabla^{2} \mathrm{~T}
$$

$\alpha=\frac{1}{\rho_{m}}\left(\frac{\partial p}{\partial T}\right)_{m}$, the thermal conductivity, $k$, the kinematic 
viscosity, $\gamma$, and the specific heat at constant pressure, $C_{p}$, are all taken to be constants. The use of the subscript $m$ and the prime is defined shortly.

Derivation of Boussinesq's Equations.

There are several ways of deriving Boussinesq's Equations. Mihaljan has used a formal perturbation expansion for a liquid. Here, a less formal method will be used for a gas, in order to exhibit the physical assumptions more clearly.

The physical variables, $P, P, T$, will be split into three parts thus

$$
E=f_{m}+E_{O}+E^{\prime} .
$$

$E_{m}$ is the value of $f$ in the middle of the fluid, at $z=0$, when there is no motion.

$f_{0}$ is the variation of $f$ from $f_{m}$ when there is no motion. $f^{\prime}$ is the variation of $f$ from its static value, $f_{m}+f_{0}$, when there is motion. $\rho=\rho(\mathrm{P}, \mathrm{T})$ is the equation of state of the gas. Expand this equation about $\rho_{\mathrm{m}}=\rho\left(\mathrm{P}_{\mathrm{m}}, \mathrm{T}_{\mathrm{m}}\right)$ in a Taylor Series. This gives

$$
\rho=\rho_{\mathrm{m}}+\left(\frac{\partial \rho}{\partial \mathrm{T}}\right)_{\mathrm{m}}\left(\mathrm{T}-\mathrm{T}_{\mathrm{m}}\right)+\left(\frac{\partial \rho}{\partial \mathrm{P}}\right)_{\mathrm{m}}\left(\mathrm{P}-\mathrm{P}_{\mathrm{m}}\right)+\cdots
$$

If we take a perfect gas

$$
P=R \rho T
$$

so that

$$
\frac{\partial p}{\partial \mathrm{T}}=-\frac{\rho}{\mathrm{T}}, \text { and } \frac{\partial \rho}{\partial \mathrm{P}}=\frac{\rho}{\mathrm{P}}
$$


If we substitute (15) in (13) we get

$$
\rho=\rho_{\mathrm{m}}\left[1-\frac{\mathrm{T}-\mathrm{T}_{\mathrm{m}}}{\mathrm{T}_{\mathrm{m}}}+\frac{\mathrm{P}-\mathrm{P}_{\mathrm{m}}}{\mathrm{P}_{\mathrm{m}}}+\cdot\right] \text {. }
$$

We now impose a restriction on the system.

$$
\frac{\Delta \rho_{0}}{\rho_{\mathrm{m}}}=\epsilon<1
$$

where $\Delta p_{0}$ is the greatest variation of $p_{0}$. We also require the dynamic fluctuations to be of the same order of magnitude, so that

$$
\left|\frac{\mathrm{T}-\mathrm{T}_{\mathrm{m}}}{\mathrm{T}_{\mathrm{m}}}\right| \leqslant \leqslant \text { and }\left|\frac{p-\mathrm{p}_{\mathrm{m}}}{\mathrm{p}_{\mathrm{m}}}\right| \leqslant \epsilon \text {. }
$$

When chere is no motion we have

$$
\begin{aligned}
\frac{\partial P_{0}}{\partial z} & =-g\left(\rho_{\mathrm{m}}+\rho_{0}\right) \\
& =-8 \rho_{\mathrm{m}}+0(\epsilon) .
\end{aligned}
$$

This, upon integrating becomes

$$
P=-8 \rho_{m^{2}}+P_{m}
$$

so that

$$
\frac{\mathrm{P}_{\mathrm{O}}}{\mathrm{P}_{\mathrm{m}}} \equiv \frac{\mathrm{P}-\mathrm{P}_{\mathrm{m}}}{\mathrm{P}_{\mathrm{m}}}=-\frac{\mathrm{gz}}{\mathrm{RT}_{\mathrm{m}}} .
$$

$\frac{\mathrm{R} \mathrm{T}_{\mathrm{m}}}{\mathrm{g}}$ has the dimensions of length, and in air at ground level has a magnitude of about $10 \mathrm{~km}$. This means that if $z$ is small compared with $10 \mathrm{~km}$

$$
\frac{P_{o}}{P_{m}} \equiv \frac{P-P_{m}}{P_{m}} \leq E \ll 1
$$


Consider the continuity equation

$$
\frac{\partial p}{\partial t}+\underline{v} \cdot \nabla \rho+\rho \nabla \cdot \underline{v}=0
$$

In the third cerm we may put $\rho \rho_{m}+o(\epsilon)$ and fn the finst two terms we may put $\rho=\rho_{0}+\rho^{\prime}$ since $\rho_{\text {m }}$ as constant. In we use (17) to put $\rho_{\mathrm{m}} \frac{\Delta \rho_{0}}{\epsilon}$, (22) becomes

$$
\epsilon\left(\frac{\partial}{\partial t}+\underline{v} \cdot \nabla\right)\left(\frac{P_{0}+p^{\prime}}{\Delta p_{0}}\right)+\nabla \cdot \underline{v}=0
$$

Therefore, as long as $\left(\frac{\partial}{\partial t}+v \cdot \nabla\right)\left(\frac{p_{0}+p^{\prime}}{\Delta \rho_{0}}\right)$ is ot the same order as $\nabla \cdot$ w we may replace (23) by $\nabla \cdot y=0$ which is (10). However, to do this we must make one furcher assumption. To clarify this we vill change to non-dimensional variables. Let

$$
\rho_{0}+\rho^{\prime}=\left(\Delta \rho_{0}\right) \hat{P}, \underline{v}=\hat{U}, t=\sigma \hat{t}, \underline{x}=\underline{\underline{x}}
$$

Then (23) becones

$$
\epsilon\left(\frac{1}{\sigma} \frac{\partial \hat{\rho}}{\partial \hat{\imath}}+\frac{u}{L} \hat{\underline{v}} \cdot \hat{\nabla} \hat{\rho}\right)+\frac{u}{L} \hat{\nabla} \cdot \hat{v}=0
$$

From (25) it can be seen that $\hat{\hat{v}} \cdot \nabla \hat{\rho}$ is of the order $\epsilon(\hat{\nabla} \cdot \hat{\underline{v}})$ but that $\frac{\partial \hat{\rho}}{\partial \hat{\varepsilon}}$ is of order $\frac{L}{\sigma} \in$ compared with $\hat{\nabla} \cdot \hat{\underline{v}}$. Therefore we make the assumption that

$$
\frac{1}{\sigma}+\frac{U}{L}
$$

(26) means that we do not allow high frequency variations in $\rho$. In other words we exclude sound waves. Thus we find that to order $\epsilon$,

$$
\nabla \cdot \underline{w}=0
$$

for the continuity equation. 
We now consider the Navier-Stokes equations,

$$
\rho \frac{\mathrm{D} \underline{\mathrm{v}}}{\mathrm{Dt}}=-\nabla \mathrm{P}-\mathrm{g} \rho \underline{\mathrm{k}}+\mu \nabla^{2} \underline{\mathrm{v}}+\frac{\mu}{3} \nabla(\nabla \cdot \mathrm{v}) .
$$

In the first term we may put $\rho=\rho_{m}$, and the last term vanishes due to (27). If we subtract the hydrostatic equation (18) we get

$$
\rho_{\mathrm{m}} \frac{\mathrm{D} \underline{\mathrm{DE}}}{\mathrm{DE}}-\nabla \mathrm{P}^{\prime}-\mathrm{g} \rho^{\prime} \underline{\mathrm{k}}+\mu \nabla^{2} \underline{\mathrm{v}} .
$$

The $z$ equation is

$$
\frac{D \omega}{D t}=-\frac{1}{\rho_{\mathrm{m}}} \frac{\partial \mathrm{p}^{\prime}}{\partial \mathrm{z}}-\frac{\mathrm{g} \rho^{\prime}}{\rho_{\mathrm{m}}}+\nu \nabla^{2} \underline{\mathrm{v}} .
$$

Consider the first two terms on the right-hand side of (30). By using (16) to transform $\frac{\rho^{\prime}}{\rho_{\mathrm{m}}}$ we find

$$
-\frac{1}{\rho_{\mathrm{m}}} \frac{\partial \mathrm{P}^{\prime}}{\partial \mathrm{z}}-\mathrm{g} \frac{\rho^{\prime}}{\rho_{\mathrm{m}}}=-\frac{1}{\rho_{\mathrm{m}}} \frac{\partial \mathrm{P}^{\prime}}{\partial \mathrm{z}}-\frac{\mathrm{gP}^{\prime}}{\mathrm{P}_{\mathrm{m}}}+g \frac{\mathrm{T}^{\prime}}{\mathrm{T}_{\mathrm{m}}} \text {. }
$$

Consider the first two terms on the right-hand side of (31). Their ratio is

$$
\frac{\mathrm{gP}^{\prime}}{\mathrm{P}_{\mathrm{m}}} / \frac{1}{\rho_{\mathrm{m}}} \frac{\partial \mathrm{P}^{\prime}}{\partial z} \sim 0\left(\frac{\mathrm{g} \mathrm{P}^{\prime}}{\mathrm{P}_{\mathrm{m}}} \cdot \frac{\rho_{\mathrm{m}} \mathrm{L}^{\prime}}{\mathrm{P}^{\prime}}\right)
$$

If we use (14) this ratio becomes $L / \frac{\mathrm{RT}_{\mathrm{m}}}{\mathrm{g}}$ which is of order $\epsilon$.

(See the argument after $(20))$. Therefore we may neglect $\frac{\mathrm{gP}^{\prime}}{\mathrm{P}_{\mathrm{m}}}$ compared with $\frac{1}{P_{m}} \frac{\partial P^{\prime}}{\partial z}$. The order of magnitude of $g \frac{T^{\prime}}{T_{m}}$ is not clear but this term must be kept since it is the driving term. 
As a result of the above argument we may take

$$
\frac{\rho^{\prime}}{\rho_{\mathrm{m}}}=-\frac{T^{\prime}}{T_{\mathrm{m}}}
$$

and the Navier-Stokes equations become, on including the hydrostatic terms,

$$
\begin{gathered}
\frac{\mathrm{Dv}}{\mathrm{Dt}}=-\frac{1}{\rho_{\mathrm{m}}} \nabla \mathrm{P}+\mathrm{g} \propto\left(\mathrm{T}-\mathrm{T}_{\mathrm{m}}\right) \mathrm{k}+\nu \nabla^{2} \underline{\mathrm{v}}, \\
\text { since } \alpha=-\frac{1}{\rho_{\mathrm{m}}}\left(\frac{\partial \rho}{\partial \mathrm{T}}\right)_{\mathrm{m}}=\frac{1}{\mathrm{~T}_{\mathrm{m}}} . \\
\text { Now, consider the energy equation, } \\
\rho \mathrm{C}_{\mathrm{v}} \frac{\mathrm{DT}}{\mathrm{Dt}}+\mathrm{P} \nabla \cdot \underline{\mathrm{v}}=\mathrm{k} \nabla^{2} \mathrm{~T}+\phi .
\end{gathered}
$$

We assume there is no internal heat source.

Although we are taking $\nabla \cdot \underline{v}=0$ we cannot ignore the term

$P \nabla \cdot \underline{v}$ since $P$ is large. To verify this we use the complete continuity equation (22) to find

$$
\mathrm{P} \nabla \cdot \underline{\mathrm{v}}=-\frac{\mathrm{P}_{\mathrm{m}}}{\rho_{\mathrm{m}}} \frac{\mathrm{D} \rho}{\mathrm{Dt}} .
$$

We then use (16) and (33) to arrive at

$$
P \nabla \cdot \underline{v}=\frac{D}{D t}\left[\begin{array}{ll}
\frac{P_{m}}{T_{m}} & T-P_{0}
\end{array}\right] \text {. }
$$

If we use (19) to substitute for $P_{0}$ and use (14) on $\frac{P_{m}}{T_{m}}$, we get

$$
\begin{aligned}
& P \nabla \cdot \underline{\mathrm{v}}=\mathrm{R} \rho_{\mathrm{m}} \frac{\mathrm{DT}}{\mathrm{Dt}}+\mathrm{gW} \rho_{\mathrm{m}} \cdot \\
& \text { If we substitute this in (35) and use }
\end{aligned}
$$

$$
\begin{aligned}
C_{\mathrm{p}}-C_{\mathrm{v}} & =\mathrm{R} \text {, we find } \\
& \rho_{\mathrm{m}} \mathrm{C}_{\mathrm{P}} \frac{\mathrm{DT}}{\mathrm{Dt}}+\mathrm{g} W \rho_{\mathrm{m}}=\mathrm{k} \nabla^{2} \mathrm{~T}+\phi .
\end{aligned}
$$


The dissipation $\phi$ is $\underline{v} \cdot\left(\mu \nabla^{2} \underline{v}\right)$. If there are to be more than just decaying solutions the driving term $g \rho^{\prime} \underline{k}$ in (29) must be the same order as $\mu \nabla^{2} \underline{v}$. This means $\phi \sim \underline{v} \cdot g \rho^{\prime} \underline{k}=g W \rho^{\prime}$. But in (36) there is a term $g W P_{m}$ and by (17) $g W P^{\prime}$ is negligible compared with it. Thus we get the third equation

$$
\frac{\mathrm{DT}}{\mathrm{Dt}}+\frac{\mathrm{gW}}{\mathrm{C}_{\mathrm{p}}}=\frac{\mathrm{K}}{\rho_{\mathrm{m}} \mathrm{C}_{\mathrm{p}}} \nabla^{2} \mathrm{~T}
$$

\section{References:}

J. M. Mihaljan, Technical Report Number 1 on Thermal Convection in Rotating Systems. Nat.ScL.Found. Feb. 1960 .

E. A. Spiegel and G. Veronis, on the Boussinesq Approximation for a Compressible Fluid. Astrophysical Journal, 1960,131, p.442. 
III. Stability Theory

Pellew and Southwell have published a paper which treats this problem exhaustively. (Proc.Roy.Soc. A. 176, 1940). However the treatment here will follow that in Jeffreys and Jeffreys (1946).

We are considering gas between two perfectly conducting, horizontal planes at $z=0$ and at $z=d$ with a temperature difference of $\Delta \mathrm{T}$ between them, the lower plane being the hotter one.

In the static case

$$
T_{0}=T_{m}+\frac{\Delta T}{d}\left(\frac{d}{2}-z\right),
$$

and

$$
\frac{\partial p}{\partial z}=-g \rho_{m}+0(\epsilon)
$$

We introduce infinitesimal fluctuations and therefore neglect squares of infinitesimal quantities in the Boussinesq equations, (9), (10) and (11). We put $g \alpha=\gamma$, subtract the hydrostatic equation from (9) and let

$$
T=T_{m}+\frac{\Delta T}{d}\left(\frac{d}{2}-z\right)+T^{\prime}
$$

Thus, (9), (10) and (11) become

$$
\begin{aligned}
& \frac{\partial \underline{v}}{\partial t}=-\frac{1}{\rho_{\mathrm{m}}} \nabla \mathrm{P}^{\prime}+\gamma \mathrm{T}^{\prime} \underline{\mathrm{k}}+\nu \nabla^{2} \underline{\mathrm{v}}, \\
& \nabla \cdot \underline{\mathrm{v}}=0 . \\
& \frac{\partial \mathrm{T}^{\prime}}{\partial \mathrm{t}}-\mathrm{W} \frac{\Delta \mathrm{T}}{\mathrm{d}}+\frac{\mathrm{g}}{\mathrm{C}_{\mathrm{P}}} \mathrm{W}=\mathrm{K} \nabla^{2} \mathrm{~T}^{\prime} .
\end{aligned}
$$

and

We define $\quad \beta=\frac{\Delta \mathrm{T}}{\mathrm{d}}-\frac{\mathrm{g}}{\mathrm{C}_{\mathrm{P}}}$

so that (43) becomes

$$
\frac{\partial T^{\prime}}{\partial t}-\beta W=k \nabla^{2} T^{\prime} .
$$


The boundary conditions are

$$
\begin{aligned}
& \mathrm{T}^{\prime}=0 \text { at both } z=0, \mathrm{~d} \\
& \underline{\mathrm{v}}=0 \text { at rigid surfaces }
\end{aligned}
$$

and for free surfaces, which we take to be flat,

$$
\begin{aligned}
& W=\frac{\partial u}{\partial z}=\frac{\partial v}{\partial z}=0 . \\
& \text { Designate the } x, y \text { and } z \text { equations of (41) as } A, B, C \text {. }
\end{aligned}
$$

Then if we take

$$
\frac{\partial^{2} \mathrm{~A}}{\partial \mathrm{x} \partial \mathrm{z}}+\frac{\partial^{2} \mathrm{~B}}{\partial \mathrm{y} z}-\nabla_{1}^{2} \mathrm{c}
$$

where $\nabla_{1}^{2}=\frac{\partial^{2}}{\partial x^{2}}+\frac{\partial^{2}}{\partial y^{2}}$, and use (42) to

$$
\text { eliminate } \frac{\partial u}{\partial x}+\frac{\partial v}{\partial y} \text { we get }
$$

$$
\left(\frac{\partial}{\partial t}-\nu \nabla^{2}\right) \nabla^{2} W=\gamma \nabla_{1}^{2} T^{\prime} \text {. }
$$

The boundary conditions are $T^{\prime}=0$, at both boundaries

$$
W=\frac{\partial^{2} W}{\partial z^{2}}=0 \text { at a free surface }
$$

(this is found by using $\frac{\partial(42)}{\partial z}$, and

$$
W=\frac{\partial W}{\partial z}=0 \text { for a rigid surface. }
$$

We put $W=e^{s t} W(x, y, z)$ and $T^{\prime}=e^{s t} T(x, y, z)$ in (45) and

(49) getting

$$
\left(s-\mathrm{k} \nabla^{2}\right) \mathrm{T}=\beta \mathrm{W}
$$

and 


$$
\left(s-\gamma \nabla^{2}\right) \nabla^{2} \mathrm{~W}=\gamma \nabla_{1}^{2} \mathrm{~T} \text {. }
$$

By taking the complex conjugate of (52) and (53) we find

$$
\left(s^{x}-k \nabla^{2}\right) T^{x}=\beta W^{x}
$$

and

$$
\left(s^{\mathrm{x}}-\nu \nabla^{2}\right) \nabla^{2} w^{\mathrm{x}}=\gamma \nabla_{1}^{2} \mathrm{~T}^{\mathrm{x}}
$$

Consider the product of (52) and (55)

$$
\beta w\left(s^{x}-\nu \nabla^{2}\right) \nabla^{2} w^{x}=\gamma \nabla_{1}^{2} T^{x}\left(s-k \nabla^{2}\right) T \text {. }
$$

We want to average this over the whole volume $V$.

Consider the term $\mathrm{W} \nabla^{2} \mathrm{~W}^{\mathrm{x}}$.

$$
\frac{1}{V} \int_{v} w \nabla^{2} w^{x} d v=-\frac{1}{v} \int_{V} \nabla \cdot w \cdot \nabla w^{x} d v+\frac{1}{v} \int_{S} w \frac{\partial w^{x}}{\partial n} d s .
$$

The last term in (57) is zero since $W=0$ on the planes $z=0$ and $z=d$, and the rest of the surface is of $O(D)$ so the whole term is of $O\left(\frac{1}{D}\right)$ which approaches zero as $D$, the horizontal dimension of the volume, approaches infinity. $\quad \frac{1}{V} \int_{V} \nabla w \cdot \nabla w^{x} d v$ is positive definite. By proceeding in this way with the other terms in (56) and doing a similar thing with (53) multiplied by (54), we deduce

$$
\begin{aligned}
& -\beta I_{1} s^{x}-\gamma \beta I_{2}+\gamma I_{3} s+x \gamma I_{4}=0 \\
& \text { and }-\beta I_{1} s-\gamma \beta I_{2}+\gamma I_{3} s^{x}+k \gamma I_{4}=0,
\end{aligned}
$$

where

$$
I_{1}=\frac{1}{v} \int_{V} \nabla w \cdot \nabla w^{x} d V
$$




$$
\begin{aligned}
& I_{2}=\frac{1}{V} \int_{V} \nabla^{2} w \nabla^{2} W^{x} d V, \\
& I_{3}=\frac{1}{V} \int_{V} \nabla_{1} T \cdot \nabla_{1} T^{x} d V,
\end{aligned}
$$

and

$$
I_{4}=\frac{1}{V} \int_{V}\left(\nabla_{1}^{2} T \nabla_{1}^{2} T^{x}+\nabla_{1} \frac{\partial T}{\partial z} \cdot \nabla_{1} \frac{\partial T^{x}}{\partial z}\right) d V .
$$

$I_{1}, I_{2}, I_{3}$ and $I_{4}$ are all positive definite.

If we put $s=p+i q$ we can find $p$ and $q$ by adding and subtracting (58) and (59). This gives

$$
p=\frac{\frac{\beta \gamma}{\gamma} I_{2}+K I_{4}}{\frac{\beta}{\gamma} I_{1}-I_{3}},
$$

and

$$
q\left(\beta I_{1}+\gamma I_{3}\right)=0
$$

(60) shows that $p$ can be zero or positive only if $\frac{\beta}{\gamma}$ is positive, on the other hand if $\frac{\beta}{\gamma}$ is positive $q=0$. Thus any amplified, or neutral disturbance 18 not periodic in time.

$$
\text { Putting } S=0 \text { in (52) and (53) yields }
$$

$$
-\mathrm{K} \nabla^{2} \mathrm{~T}=\beta \mathrm{W}
$$

and

$$
-\nu \nabla^{4} \mathrm{~W}=\gamma \nabla_{1}^{2} \mathrm{~T}
$$

so that

$$
\nabla^{6} \psi=\frac{\gamma \beta}{k} \nabla_{1}^{2} \psi
$$

where $\psi=$ wor $\mathrm{T}$.

$$
\begin{aligned}
& \text { Let } \quad T=f(x, y) \theta(z) \\
& \text { and } \quad W=g(x, y) h(z)
\end{aligned}
$$


We assume that $g$ and $f$ are functions of $x, y$ such that

$$
\nabla_{1}^{2} E=-\frac{\pi^{2} \alpha^{2}}{d^{2}} f . \quad(f=g)
$$

We shall put $z=\frac{d J}{\pi}$, so that $J$ goes from 0 to $\pi$ as $z$ goes from 0 to $d$, and (64) becones

$$
\left(\frac{d^{2}}{d J^{2}}-\alpha^{2}\right)^{3} \psi=-\frac{\gamma \beta d^{4} \alpha^{2}}{k \nu \pi^{4}} \psi \text {. }
$$

We whall put $\frac{\gamma \beta d^{4}}{k \nu \pi^{4}}=R^{\prime}=\frac{\text { Rayleigh number }}{\pi^{4}}$.

By considering (50), (62) and (63), the boundary conditions at a free surface are seen to be

$$
\psi=\frac{\partial^{2} \psi}{\partial J^{2}}=\frac{\partial^{4} \psi}{\partial y^{4}}=\cdots=0 .
$$

Similarly by using (51), (62) and (63) the boundary conditions at

a rigid surface are

$$
T=\frac{\partial^{2} T}{\partial \zeta^{2}}=\frac{\pi^{2}}{d^{2}} \frac{\partial^{3} T}{\partial \zeta^{3}}+\nabla_{1}^{2} \frac{\partial T}{\partial \zeta}=0,
$$

and $W=\frac{\partial W}{\partial \zeta}=\frac{\pi^{2}}{d^{2}} \frac{\partial^{4} W}{\partial \zeta^{4}}+2 \nabla_{1}^{2} \frac{\partial^{2} W}{\partial \zeta^{2}}=0$.

If we substitute for $T$ and $W$, and use (65) in (68) and (69) we have for a rigid surface, which is the case we will consider,

$$
\theta=\theta^{\prime \prime}=\theta^{\prime \prime \prime}-\alpha^{2} \theta^{\prime}=0 \text {, }
$$

and $h^{\prime}=h^{\prime}=h^{\prime v}-2 \alpha^{2} h^{\prime \prime}=0$

on the boundaries. 
We shall consider the equation for $\theta$,

$$
\left(\frac{d^{2}}{d J^{2}}-\alpha^{2}\right)^{3} \theta=-R^{\prime} \alpha^{2} \theta \text {. }
$$

We now want to substitute a Fourier sine series for $\theta$ but we must first make a digression on differentiating Fourier series.

\section{Digression on Fourier series.}

If we take a Fourier cosine series of a function $f$,

$$
f=a_{0}+\sum_{i}^{\infty} a_{n} \cos n J \text {, }
$$

differentiate,

$$
f^{\prime}=-\sum_{1}^{\infty} n a_{n} \sin n J
$$

and compare this with the Fourler sine series of $f^{\prime}$

$$
f^{\prime}=\sum_{j}^{\infty} b_{n} \sin n J
$$

where $b_{\mathfrak{n}}$ are evaluated thus

$$
\begin{gathered}
b_{n}=\frac{2}{\pi} \int_{0}^{\pi} f^{i} \sin n J d J=\frac{2}{\pi}[f \sin n J]_{0}^{\pi}-\frac{2}{\pi} \int_{0}^{\pi} n f \cos n J d J \\
=0-n a_{n} .
\end{gathered}
$$

Therefore, the Fourter cosine series of $f$ gives the Fourier sine series of $E^{\prime}$ when it is differentiated.

On the other hand if we take the Fourier sine series of

a function $f$

$$
f=\sum_{1}^{\infty} a_{n} \sin n J
$$

differentiate,

$$
\left.f^{\prime}=\sum_{1}^{\infty} n a_{n} \cos n\right\}
$$

and then compare this with the fourier cosine series of $f^{\prime}$ 
$-26-$

$$
\begin{gathered}
f^{\prime}=b_{0}+\sum_{1}^{\infty} b_{n} \cos n J \\
\text { where } b_{0}=\frac{1}{\pi} \int_{0+}^{f^{\prime} d} J=\frac{1}{\pi}[f(\pi-)-f(0+)] \\
\text { and } b_{n}=\frac{2}{\pi} \int_{0+}^{\pi-} f^{\prime} \cos n J J=\frac{2}{\pi}[f \cos n J]_{0+}^{\pi+}+\frac{2}{\pi} \int_{0}^{\pi} n E \sin n J_{d} J \\
=\frac{2}{\pi}\left[(-1)^{n} f(\pi-)-f(0+)\right]+n a_{n},
\end{gathered}
$$

we find that

$$
\left.f^{\prime} \neq \sum_{1}^{\infty} n a_{n} \cos n\right\}
$$

unless $f(0+)=f\left(\pi_{-}\right)$. This means that we may need to add terms to a differentiated fourier sine series of $f$ if $i t$ is to be the fourier cosine series of $\mathrm{f}^{\prime}$.

We now put

$$
\left.\theta=\sum_{1}^{\infty} A_{n} \sin n\right\} \text {. }
$$

$\theta$, and $\theta^{\prime \prime}$ are both zero at $0, \pi$ so we may differentiate them without trouble. Therefore

$$
\begin{aligned}
& \theta^{\prime}=\sum_{1}^{\infty} n A_{n} \cos n J, \quad \theta^{\prime \prime}=-\sum_{1}^{\infty} n^{2} A_{n} \sin n J, \\
& \theta^{\prime \prime \prime}=-\sum_{1}^{\infty} n^{3} A_{n} \cos n J, \quad \theta^{\prime v}=\sum_{1}^{\infty} n^{4} A_{n} \sin n J .
\end{aligned}
$$

$\theta^{I V}(0)$ and $\theta^{I V}(\pi)$ do not necessarily equal zero, so we must put

$$
\left.\theta^{v}=\frac{1}{\pi}\left[\theta^{i v}(\pi-)-\theta^{i v}(0+)\right]+\sum_{i}^{\infty}\left(n^{b} A_{n}+{ }_{B}^{A}\right) \cos n\right\},
$$

where $A=\frac{2}{\pi}\left[-\theta^{i v}(\pi-)-\theta^{i v}(0+)\right]$ for odd $n$

and $B=\frac{2}{\pi}\left[\theta^{W}(\pi-)-\theta^{V}(0+)\right]$ for even $n$

so

$$
\theta^{v \prime}=-\sum_{1}^{\infty}\left(n^{6} A_{n}-n_{B}^{A}\right) \sin n J \text {. }
$$


We now substitute (73), (74), (75) and (76) in (72) and get,

$-\sum_{i}^{\infty}\left(n^{2}+\alpha^{2}\right)^{3} A_{n} \sin n \zeta-\sum_{i}^{\infty} A \sin n J+\sum_{i}^{\infty} R^{\prime} \alpha^{2} A_{n} \sin n J=0$.

If we multiply (77) by sin $n J$ and integrate from 0 to $\pi$ we see

that

$$
-\left(n^{2}+\alpha^{2}\right)^{3} A_{n}+R^{\prime} \alpha^{2} A_{n}=n A \text { for odd } n
$$

and

$$
-\left(n^{2}+\alpha^{2}\right)^{3} A_{n}+R^{\prime} \alpha^{2} A_{n}=n B \text { for even } n \text {. }
$$

The boundary condition (70) at $J=0$ is

$$
-\sum_{1}^{\infty} n^{3} A_{n}-\alpha^{2} \sum_{i}^{\infty} n A_{n}=0
$$

and for $\zeta=\pi$

$$
\begin{aligned}
& -\sum_{1}^{\infty} n^{3}(-1)^{n} \cdot A_{n}-\alpha^{2} \sum_{1}^{\infty} n(-1)^{n} A_{n}=0 . \\
& \text { Adding (80) and (81), we find } \\
& \sum_{\text {even } n}^{\infty}\left(n^{2}+\alpha^{2}\right) n A_{n}=0,
\end{aligned}
$$

and if we subtract (80) from (81)

$$
\sum_{\text {odd } n}^{\infty}\left(n^{2}+\alpha^{2}\right) n A_{n}=0 .
$$

We shall consider odd terms only, now, since they are those which are symetrical about the center. It may be proved by considering the other terms, that a symmetrical mode would be the least stable,

Therefore, we use (78) and (83) to eliminate $A_{n}$

$$
\sum_{\text {odd } n} \frac{\left(n^{2}+\alpha^{2}\right) n^{2} A}{\left(n^{2}+\alpha^{2}\right)^{3}-R^{\prime} \alpha^{2}}=0
$$


By manipulating (84) thus

$$
\sum_{\text {odd } n}\left[\frac{\left.n^{2}+\alpha^{2}\right) n^{2}}{\left(n^{2}+\alpha^{2}\right)^{3}-R^{\prime} \alpha^{2}}-\frac{n^{2}}{\left(n^{2}+\alpha^{2}\right)^{2}}+\frac{n^{2}}{\left(n^{2}+\alpha^{2}\right)^{2}}\right]=0
$$

we find

$$
\sum_{\text {odd } n} \frac{n^{2} \alpha^{2} R^{\prime}}{\left(n^{2}+\alpha^{2}\right)^{2}\left[\left(n^{2}+\alpha^{2}\right)^{3}-R^{\prime} \alpha^{2}\right]}+\sum_{\text {odd } n} \frac{n^{2}}{\left(n^{2}+\alpha^{2}\right)^{2}}=0 \text {. }
$$

(85) is convenient for manipulation since the terms of the first series are of order $\frac{1}{n}$, making the series converge rapidly, and Jeffreys pointed out that

$$
\begin{gathered}
\tanh \frac{\pi \alpha}{2}=\frac{4 \alpha}{\pi} \sum_{\text {odd } n} \frac{1}{n^{2}+\alpha^{2}}, \\
\frac{d}{d \alpha}\left(\tanh \frac{\pi \alpha}{2}\right)=\frac{4}{\pi} \sum_{\text {odd } n} \frac{1}{n^{2}+\alpha^{2}}-\frac{4 \alpha}{\pi} \sum_{\text {odd } n} \frac{2 \alpha}{\left(n^{2}+\alpha^{2}\right)^{2}}
\end{gathered}
$$

so that

$$
\sum_{\text {odd } n} \frac{n^{2}}{\left(n^{2}+\alpha^{2}\right)^{2}}=\frac{\pi}{8}\left(\frac{d}{d \alpha}+\frac{1}{\alpha}\right) \tanh \frac{\pi \alpha}{2}=T \alpha .
$$

Putting (86) in (85) yields

$$
\frac{R^{\prime} \alpha^{2}}{\left(1+\alpha^{2}\right)^{2}\left[\left(1+\alpha^{2}\right)^{3}-R^{\prime} \alpha^{2}\right]}+T \alpha+K=0
$$

where

$$
K=\sum_{\substack{n=3 \\ \text { odd } n}}^{\infty} \frac{n^{2} \alpha^{2} R^{\prime}}{\left.\left(n^{2}+\alpha\right)^{2}\right)^{2}\left[\left(n^{2}+\alpha^{2}\right)^{3}-R^{\prime} \alpha^{2}\right]}
$$

We can compute the minimum value of $R^{\prime}$ with $K=0$ and then put this value of $R^{\prime}$ in $K$ and 1terate. Jeffreys does this with one 
iteration and finds the minimum Rayleigh number $=1708.2$ for $\alpha=.995$. Pellew and Southwell compute $R=1707.8$ for $\alpha=.993$ from the exact eigenfunctions. Jeffreys' method is much simpler computationally.

A variational method for computing $\mathrm{R}_{c}$ Equation (66) can be written, with $a^{2}=\frac{\pi^{2} \alpha^{2}}{d^{2}}$,

$$
\left(\frac{d^{2}}{d z^{2}}-a^{2}\right)^{3} w+R \frac{a^{2} w}{d^{4}}=0 \text {, }
$$

where $W$ is that part of the vertical velocity which depends only on $z$, and $R$ is the Rayleigh number. We multiply (87) by $\left(\frac{d^{2}}{d z^{2}}-a^{2}\right)^{2} W$ (which is proportional to $T$ by $(63)$ ) and integrate by parts from o to $d$, to get

$$
R=\frac{d^{4}}{a^{2}} \frac{\int_{0}^{d}\left\{\left[\frac{d}{d z}\left(\frac{d^{2}}{d z^{2}}-a^{2}\right)^{2} w\right]^{2}+a^{2}\left[\left(\frac{d^{2}}{d z^{2}}-a^{2}\right)^{2} w\right]^{2}\right\} d z}{\int_{0}^{d}\left[\left(\frac{d^{2}}{d z^{2}}-a^{2}\right) W\right]^{2} d z} .
$$

(88) is positive definite.

The variation of $R$ is

$$
\delta R=\frac{-\frac{2}{a^{2}}}{a^{2} \int_{0}^{d}\left[\left(\frac{d^{2}}{d z^{2}}-a^{2}\right) w\right]^{2} d z} \cdot \int_{0}^{d} \delta\left[\left(\frac{d^{2}}{d z^{2}}-a^{2}\right) W\right]\left\{\left(\frac{d^{2}}{d z^{2}}-a^{2}\right)^{3} W+R \frac{a^{2}}{d^{4}} W\right\} d z .
$$

Hence $\delta_{R}=0$ if $W$ is a solution of (87). Also the expression on the right-hand side of (88) is minimized by inserting for $W$ a solution of (87) satisfying the boundary conditions. Since 
we are now concerned with findingthe minimum value of $R$ for which there is a solution $W$, the right-hand side of ( 88$)$, when minimized with respect to $a$, wlll provide an estimate for $R_{c}$, when an approximation for $W$ is inserted, as explained directly below.

The fact that the right-hand side of (88) is stationary for solutions $W$ means that if any function within order $E$ of a solution of (87) but satisfying the boundary conditions exactly, is inserted in (88), then the minimum value of $R$ obtained will differ from the correct value $R_{c}$ by $O\left(\epsilon^{2}\right)$. In this problem it 18 better to "guess" at $\left(\frac{d^{2}}{d z^{2}}-a^{2}\right)^{2} W$, than to guess W directly. Using

$$
\left(\frac{d^{2}}{d z^{2}}-a^{2}\right)^{2} w=1-\cos 2 \pi z-a \sin \pi z
$$

Pellew and Southwell found the following estimates, for different wave numbers ad, when minimized with respect to $A$,

\begin{tabular}{|c|c|c|c|}
\hline \multirow{5}{*}{ a d } & & Exact $\mathrm{R}$ & Approx. $\mathrm{R}$ \\
\hline & $=$ & 2177.4 & 2177.6 \\
\hline & 3 & 1711.3 & 1711.4 \\
\hline & 4 & 1879.3 & 1879.35 \\
\hline & 5 & 2439.4 & 2439.4 \\
\hline
\end{tabular}

The actual minimum 18 at ad $=3.12$, at which the exact value of $R$ $18 R_{c}=1707.8$ 
IV. $\underline{\text { Ce11s }}$

Recall that the differential equation satisfied by $f(x, y)$, the "horizontal" part of the vertical velocity, is, (65)

$$
\nabla_{1}^{2} \mathrm{f}+\frac{\alpha^{2} \pi^{2}}{\mathrm{~d}^{2}} \mathrm{f}=0 .
$$

As we have stated previously at the point of instability, motion is realized as a regular cellular pattern in the horizontal plane. That is, looking down on the fluid from above one might see an array of cells of which the following diagram is an example.

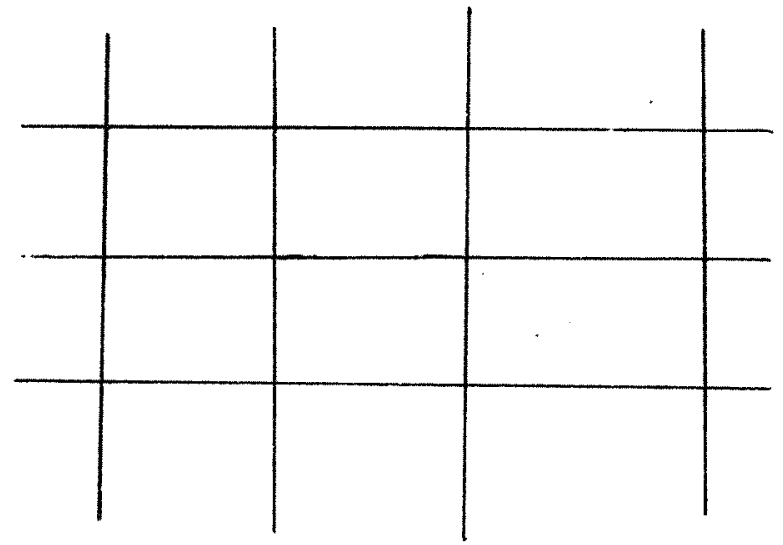

Convection takes place in each cell separately, that is, the fluid in one cell moves only in that cell so that $\underline{v} \cdot \underline{n}=0$ on the boundary of the cell. The motion is either, up at the centre of the ce11 and down at the edges, or the other way around. The shape of the cells in an array is called a "plan-form", and clearly $f$ can be identified with the plan-form of a motion.

Bisshopp (1961) performed a careful analysis of possible plan-forms (see also Pellew and Southwe11) and showed that only "rolls", rectangles, squares, triangles and hexagons are permissible. 
Ro11s are long strips

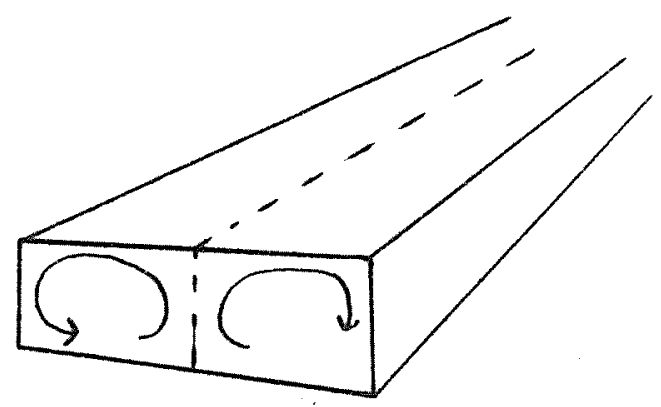

He shows that all close-packed tesselations are allowed except for parallelograms.

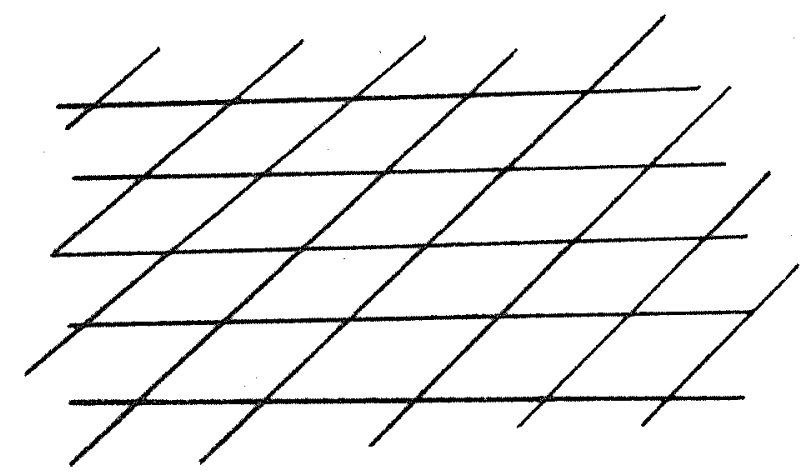

Pellew and Southwell speculate that the non-1inear terms in the equations of motion should determine which cell shapes actually occur. The extent to which this is true will be seen in the study of the finite-amplitude problem, to which we shall soon direct our attention.

While $R_{c}$ does not depend on cell shape, other flow quantities, such as the heat transport, vary a great deal with cell shape. Indeed, the variation of heat transport provides much of the motivation for studying the problem of preferred cell shapes. submitted by Howell Peregrine and Steve Rosencrans. 


\section{Binite Amplitude (non-Iinear) Theory}

\section{Introduction}

The main value of the linearized theory is its comparative simplicity. Unfortunately its validity is severely limited. Mathematically, it is valid only when the departure of the system from a solution of the full equations is infinitesimal. If the basic state is a state of conduction (no motion), then this restriction will hold only in the sub-crittcal range. When the Rayleigh number is increased above the critical value, a disturbance will grow not without linit, but only until the non-linear interactions become significant and cause the system to relax in a new, statistically steady state of motion. Furthermore, even when the linearized theory predicts stability, the result is not guaranteed. Veronis has shown that in some (admittedly few) cases, a system in a state which is stable with respect to infinitesimal disturbances may really be metastable, and eventually may experience a disturbance large enough to cause it to go over into a preferred state of motion. The finite amplitude analysis (lectures VI and VII) wIII enable us to calculate forms of motion which may exist when $R>R_{c r i t}$ and the state of zero motion can not exist.

The Idea of a preferred state of motion presents another problem, which will be discussed in lecture VIII. Finite amplitude analysis will show that there are infintely many statistically steady forms of motion avallable to the system in the convective range. How can we decide which state the system actually picks, without attempting to treat an intractible stability problem? 
There is an analogy to the problem of a single point mass, subject to dissipative forces, randon "noise", and a fixed potential field. In this case we have a simple criterion: the mass will eventually settle into the state of lowest potential energy and spend most of its time there,* In lecture VII we will derive, subject to certain hypotheses, a criterion to compare the "relative stability" of different allowed states of motion. This will lead to a simple principle: the system will tend toward the state which permits the most heat transport.

We write for the total temperature

$$
T(x, y, z, t)=T_{m}+\bar{T}(z, t)+T(x, y, z, t)
$$

where

$$
\bar{T}=\left(T-T_{\mathrm{m}}\right)_{\text {horlzontal average }}
$$

Thus eq (1) defines $T$ as a fluctuation.

The horizontal average is equivalent to the statistical ensemble average (for fixed $z$ ). The bar, in fact, will always denote an average over horizontal directions

$$
\bar{F}(z)=\lim _{a \rightarrow \infty} \frac{1}{4 a^{2}} \int_{-a}^{a} d y \int_{-a}^{a} d x F(x, y, z)
$$

note: $\bar{T}=0$ by definition

We shall frequently make use of the following:

$$
\text { a) } \frac{\overline{\partial F}}{\partial x}=\frac{\overline{\partial F}}{\partial y}=0
$$

*Consider a billiard ball lying in a metastable state on the blliiard table. Eventually, it will wind up in a crack in the floor, as a simple experiment will show. 
where $F$ is any bounded function with a finite number of discontinuities. This follows from the definition of the horizontal average. Also :

$$
\begin{aligned}
& \text { b) } \bar{w}=0 \text { because } \\
& 0=\frac{\partial u}{\partial x}+\frac{\partial v}{\partial y}+\frac{\partial w}{\partial z}=\frac{\partial u}{\partial x}+\frac{\partial v}{\partial y}+\frac{\partial w}{\partial z}=\frac{\bar{w}}{z},
\end{aligned}
$$

so $\bar{w}$ is constant and equal to its boundary value which is 0 .

The double bar will denote an average taken over the entire configuration

$$
\overline{\bar{F}}=\frac{1}{d} \int_{0}^{d} \bar{F}(z) d z
$$

Constraints on the motion:

We start with the heat equation:

$$
\begin{array}{r}
\left(\frac{\partial}{\partial t}-k \nabla^{2}\right)\left(T-T_{m}\right)=-\vec{v} \cdot \vec{\nabla}\left(T-T_{m}\right)= \\
-\vec{\nabla} \cdot\left[\vec{v}\left(T-T_{m}\right)\right] \\
(\operatorname{since} \vec{\nabla} \cdot \vec{v}=0)
\end{array}
$$

taking the horizontal average yields

$$
-K \frac{\partial^{2} \bar{T}}{\partial z^{2}}=-\frac{\partial}{\partial z}(\overline{W T})
$$

where we have set $\frac{\partial \bar{T}}{\partial t}=0$ in view of the steady state.

We may integrate once to obtain

$$
\begin{aligned}
& \text { (after defining } \left.\beta(z)=-\frac{\partial \bar{T}}{\partial z}\right) \\
& \mathrm{K} \beta+\overline{\mathrm{WT}}=\mathrm{H} \\
& \text { conducted convected total kinematic } \\
& \text { heat flux heat flux heat flux (a constant) }
\end{aligned}
$$

Averaging over $z$ we get

$$
\mathrm{K} \beta_{\mathrm{m}}+\overline{\overline{\mathrm{WT}}}=\mathrm{H}
$$

where

$$
\beta_{m}=\frac{1}{d} \int_{0}^{d} \beta d z=\frac{\Delta \bar{T}}{d}
$$


Then

$$
\frac{\beta}{\beta_{m}}=1+\frac{\overline{\overline{W T}}-\overline{W T}}{k \beta_{m}}
$$

Then since $T-T_{m}=\bar{T}+T$, we may write the heat equation (1) in terms of the fluctuating quantities after using (9), (10) and (11)

$$
\left(\frac{\partial}{\partial t}-k \nabla^{2}\right) T=\beta W-\left(V \cdot \nabla T-\frac{\partial}{\partial z}(W T)\right)=\beta W-h
$$

where $h \equiv v \cdot \nabla T-\frac{\partial}{\partial z}(\overline{W T})$ incorporates the zero-average nonlinear terms.

i.e. note that

$$
\bar{h}=\overline{\nabla \cdot \bar{v} T}-\frac{\partial}{\partial z}(\overline{W T})=\overline{\frac{\partial}{\partial} W T}-\frac{\partial}{\partial z} \overline{W T}=0
$$

For convenience we write down again the N-S equation:

$$
\left(\frac{\partial}{\partial t}-\nu \nabla^{2}\right) \vec{v}=\gamma(\bar{T}+T) \hat{K}-\frac{\nabla \vec{\rho}}{\rho_{m}}-\vec{v} \cdot \vec{\nabla} \vec{v}
$$

Further Constraints: The power Integrals.

$$
\text { First note that } \overline{\overline{h T}}=0
$$

proof:

$$
\left.\overline{h T}=\overline{T \nabla \cdot(\vec{v} T)}-\left(\frac{\partial}{\partial \cdot(W T}\right)\right) \bar{T}=0
$$

Now

$$
\text { Now } \begin{aligned}
T \nabla \cdot(\vec{v} T)=T[T \vec{\nabla} \cdot \vec{v}+\vec{v} \cdot \nabla T] & =\frac{\vec{v}}{2} \cdot \vec{\nabla}\left(T^{2}\right)=\nabla \cdot\left(T^{2} \vec{v}\right) \\
\text { So } & \overline{h T}=\overline{\nabla \cdot\left(\frac{T^{2} \vec{v}}{2}\right)}=\frac{\partial}{\partial z}\left(\frac{T^{2} W}{2}\right)
\end{aligned}
$$

Therefore $\overline{\overline{h T}}=0$ since both $\mathrm{T}$ and $W$ vanish at $z=0, d$. Multiplying (16) by $T$ and taking the complete average we get

$$
\frac{1}{2} \frac{\partial}{\partial t} \overline{\overline{T^{2}}}=\overline{\beta \overline{W T}}-K \overline{\bar{\nabla} T \cdot \bar{\nabla} T}
$$

where the last term has been symmetrized by integration by parts. 
Similarly, we may take the scalar product of (18) with $\vec{v}$ and average to get

$$
\frac{1}{2} \frac{\partial}{\partial t} \overline{\overline{(\vec{v} \cdot \vec{v})}}=\gamma \overline{\overline{W T}}-\nu \overline{\frac{\partial v_{i}}{\partial x_{j}} \frac{\partial v_{i}}{\partial x_{j}}}
$$

Now, in the statistically steady state the averaged quantities will be time independent so, after using (15) to elfminate $\beta$, we obtain

$$
\begin{gathered}
\rho_{m} \overline{\overline{W T}}+\frac{1}{K}(\overline{\bar{W} T})^{2}-\frac{1}{k(\overline{W T})^{2}}-k \overline{\overline{\bar{\nabla} T \cdot \vec{\nabla} T}}=0 \\
\gamma \overline{\overline{W T}}-\nu \overline{\frac{\partial v_{i}}{\partial x_{j}} \frac{\partial v_{i}}{\partial x_{j}}}=0
\end{gathered}
$$

These are the "power integrals". Since they are inhomogeneous in $W$ and $T$, they provide a restriction on the amplitude of the fluctuating quantities. I.e. If we somehow knew everything about the fluctuating quantities except the amplitude, then the amplitude is determined immediately from the power integrals. For example we may take for $\vec{u}$ and $T$ the marginally stable solutions of the linearized problem. (No claim is made of course that they are the correct solutions for $\left.\mathrm{R}>\mathrm{R}_{\mathrm{c}} \cdot\right)$

satisfies

$$
\begin{aligned}
& w=f(x, y) \sin \pi z / d \quad \text { and } f(x, y) \\
& \nabla_{1}^{2} f(x, y)+\frac{\alpha^{2} \pi^{2}}{d^{2}} f(x, y)=0 \\
& T=\frac{\nu \pi^{2}}{d^{2}} \frac{\left(1+\alpha^{2}\right)}{\gamma \alpha^{2}} w \\
& u=\frac{-d^{2}}{\pi^{2} \alpha^{2}} \frac{\partial^{2} w}{\partial x \partial z} \\
& v=-\frac{d^{2}}{\pi^{2} \alpha^{2}} \frac{\partial^{2} w}{\partial y \partial z}
\end{aligned}
$$


Since we expect cellular motion we choose (just to see what happens)

$$
f=2 \mathrm{~A} \cos (\alpha \pi x / d) \text { (the roll form) }
$$

A is the unknown amplitude.

When we insert these expressions for the form of the fluctuating quantities in the power integrals and determine $A$, and then evaluate WT (the convected heat) we obtain

$$
\overline{\overline{W T}}=k\left(\beta_{m}-\beta_{m}^{c r i t .}\right) \text { where } \beta_{m}^{c r i t} \text { is } \beta_{m} \text { when } R=R \text { crit }
$$

This gives a slope in the $\mathrm{R}-\mathrm{N}_{\mathrm{u}}$ curve of 2 . Use of the rigid boundary marginal solutions in (24) and (25) gives a slope of 1.51 in agreement with experiment (see page 10).

The Finite Amplitude Expanston:

Let us apply the operator

$$
\theta=\hat{i} \frac{\partial^{2}}{\partial x \partial z}+\hat{\jmath} \frac{\partial^{2}}{\partial y \partial z}-\hat{k}\left(\frac{\partial^{2}}{\partial x^{2}}+\frac{\partial^{2}}{\partial y^{2}}\right)
$$

to the N-S equation (18).

$$
\begin{aligned}
& \text { Note } \theta \cdot \vec{\nabla} \equiv 0 \text { which eliminates the } \vec{\nabla} p \text { term . } \\
& \text { Also, } \theta \cdot \vec{v}=\frac{\partial}{\partial z} \frac{\partial u}{\partial x}+\frac{\partial}{\partial z} \frac{\partial u}{\partial y}-\left(\frac{\partial^{2}}{\partial x^{2}}+\frac{\partial^{2}}{\partial y^{2}}\right) w \\
& =\frac{\partial}{\partial z}\left[\begin{array}{c}
\left.\vec{\nabla} \cdot \vec{v}-\frac{\partial w}{\partial z}\right]-\left(\frac{\partial^{2}}{\partial x^{2}}+\frac{\partial^{2}}{\partial y^{2}}\right) w=-\nabla^{2} w
\end{array}\right.
\end{aligned}
$$

Thus

$$
\begin{aligned}
& \left(\frac{\partial}{\partial t}-v \nabla^{2}\right) \nabla^{2} w=\gamma \nabla_{1}^{2} T+L \\
& \text { where } L=\theta \cdot[\vec{v} \cdot \vec{\nabla} \vec{v}]
\end{aligned}
$$


We may eliminate $T$ by writing

$$
\gamma \nabla_{1}^{2} T=-\left(\frac{\partial}{\partial t}-\nu \nabla^{2}\right) \nabla^{2} \omega+L
$$

and inserting in (36)

the heat equation (16), obtaining the $6^{\text {th }}$ order equation satisfied .

by w.

$$
\left(\frac{\partial}{\partial t}-k \nabla^{2}\right)\left(\frac{\partial}{\partial t}-\nu \nabla^{2}\right) \nabla^{2} w-\gamma \nabla_{1}^{2} \beta w=\left(\frac{\partial}{\partial t}-k \nabla^{2}\right) L-\gamma \nabla_{1}^{2} h
$$

The basic equations will be (36) and (38). For simplicity we "non-dimensionalize" them according to

$$
\vec{v} \rightarrow \frac{k}{d} \vec{v}^{\prime} \quad T \rightarrow \frac{\nu k}{r d^{3}} T^{\prime} \vec{x} \rightarrow d \vec{x}^{\prime} \quad t \rightarrow \frac{d^{2}}{k} t^{\prime}
$$

to give

$$
\begin{gathered}
\left(\frac{1}{\sigma} \frac{\partial}{\partial t}-\nabla^{2}\right) \nabla^{2} w=\nabla_{1}^{2} T+\frac{1}{\sigma} L \\
\left(\frac{1}{\sigma} \frac{\partial}{\partial t}-\nabla^{2}\right)\left(\frac{\partial}{\partial t}-\nabla^{2}\right) \nabla^{2} w-R \nabla_{1}^{2} w=\left(\frac{1}{\sigma} \frac{\partial}{\partial t}-\nabla^{2}\right) L-\nabla_{1}^{2} h+(\overline{W T}-\overline{W T}) \nabla_{1}^{2} w
\end{gathered}
$$

where we have used (15), .. in order to put all non-1inear terms on the right-hand side. The characteristics of the system influence the problem only through the Rayleigh number and the Prandt 1 number $\sigma$ where

$$
\sigma=2 / k
$$

We shall try to satisfy $(40)$ and $(41)$ by means of a formal expansion

$$
\begin{aligned}
& W=\sum_{0}^{\infty} w_{i} e^{i+1} \\
& T=\sum_{0}^{\infty} T i \epsilon^{i+1}
\end{aligned}
$$


and we assume that these expressions converge for $\epsilon$ in some neighborhood of the origin. No particular physical meaning is given to $\epsilon$; however the power integral (24) may be written

$$
R=\frac{\overline{T\left(\frac{\partial^{2}}{\partial t^{2}}-\nabla^{2}\right) T}-\overline{[(\overline{\overline{W T}}-\overline{W T}) \overline{W T}]}}{\overline{\bar{W}}}
$$

(after applying $\left(\beta-\beta_{m}\right)=\frac{1}{K}(\overline{\overline{W T}}-\overline{W T})$ and non-dimensionalizing).

Then we see that $R$ wil appear also as a power series in $\epsilon$

$$
R=\sum_{0}^{\infty} R_{i} \epsilon^{i}
$$

Another way of saying this is that the expansions (42) and (43) of $W$ and $T$ do not provide a consiatent means of satiafying (40) and (41) unless $R$ is taken also as being explicitly dependent upon the parameter $\epsilon$. Of course this is not a real disadvantage. The number $E$ will determine both the $W-T$ fleld and the number $R$, the value of the physical parameters for which that $W-T$ field is a solution of the equations:

The hierarchy of equations is:

$$
\begin{aligned}
& \mathcal{L} w_{0}=\left(\frac{\partial}{\partial t}-\nabla^{2}\right)\left(\frac{1}{\sigma} \frac{\partial}{\partial t}-\nabla^{2}\right) \nabla^{2} w_{0}-R_{0} \nabla_{1}^{2} w_{0} \\
&= 0 \\
& \mathcal{L} w_{1}= R_{1} \nabla_{1}^{2} w_{0}-\nabla_{1}^{2} h_{00}+\frac{1}{\sigma}\left(\frac{\partial}{\partial t}-\nabla^{2}\right) L_{00} \\
& \mathcal{L} w_{2}=R_{1} \nabla_{1}^{2 m} w_{1}+R_{2} \nabla_{1}^{2} w_{0}+\left\{\overline{\overline{w_{0}^{\prime} T_{0}}}-\overline{w_{0} T_{0}}\right\} \nabla_{1}^{2} w_{0}-\nabla_{1}^{2}\left(h_{10}+h_{01}\right)+ \\
&+\frac{1}{\sigma}\left(\frac{\partial}{\partial t}-\nabla^{2}\right)\left(L_{0}+L_{10}\right)
\end{aligned}
$$


where $h_{i j}=h\left(w_{i}, T_{j}\right)$ and similarly for $L_{i j}$.

One may also write expressions for $T, u, v$.

Although we may not write it explicitly, we will assume that the $\vec{u}_{0}, T_{0}$ fields are of the form

$$
\begin{aligned}
\vec{\nu}_{0}, T_{0} & =\phi F(z, t) \text { with } \\
\nabla_{1}^{2} \phi & =-\alpha^{2} \pi^{2} \phi
\end{aligned}
$$

Now, If we require statistically sceady solutions - i.e. it we require that horizontally averaged quantities are time independent then the $\vec{u}$, T fields themselves will have to be time independent in order to satisfy $(46)_{n}$. In this case, equation (46) $)_{0}$ is simply the equation for marginal stability (see page 23), and $R_{0}$ will be the critical value of $\mathrm{R}$ for the given $\alpha$. There are still a few other problems. First of all we note that $\omega_{0} \mathscr{L} w_{i}$ must vanish because $\overline{\overline{\omega_{0} \mathcal{L} w}}=\overline{\overline{\omega_{i} \mathcal{L} w_{0}}}$ (48). since $\mathcal{L}$ is hermitian with respect to the free boundary conditions. Thus $W_{0}$ must be orthogonal to the right-hand side of all equations in the hierarchy. This condition, besides insuring that the equations possess solutions, also determines the $\mathrm{R}_{i}$. For example, taking the mean of $w_{0}$ times eq. (46)' gives

$$
R_{1}=\frac{\overline{W_{0} \nabla_{1}^{2} h_{00}}+\frac{1}{\sigma} \overline{W_{0} \nabla^{2} L_{00}}}{\overline{W_{0} \nabla_{1}^{2} W_{0}}}
$$

There is still an indeterminacy in the solution since we could add to any of the $W_{i}$ a multiple of $W_{0}$, the solution of the homogeneous 
equation $(46)^{\circ}$. We resolve this by requiring

$$
\overline{\overline{w_{0} w_{i}}}=0 i \neq 0
$$

Actual computations require a choice of plan form (an explicit expression for $\phi(x, y)$ ).

Examples:

$$
\text { Rolls } \phi=2 \cos \pi \alpha x
$$

$$
\text { Rectangles } \phi=2 \sqrt{2} \cos \pi \ell x \cos \pi m y \leftarrow \ell^{2}+m^{2}=\alpha^{2}
$$

Hexagons $\phi=\frac{2}{\sqrt{3}}\left(2 \cos \frac{2 \pi x}{\sqrt{3} L} \cos \frac{2 \pi y}{3 L}+\frac{\cos 4 \pi y}{3 L}\right)$

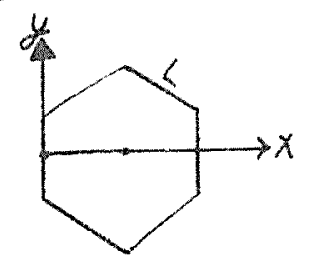

$$
\text { The amplitudes are chosen so that } \overline{\overline{W_{0}^{2}}}=1
$$

For rolls we directly compute

$$
h_{00}=0 \quad L_{00}=0
$$

which implies $R_{1}=0$

which in turn implies $\omega_{1}=T_{1}=u_{1}=v_{1}=0$

so $\quad h_{01}=h_{10}=L_{01}=L_{10}=0$

and $\mathcal{L} W_{2}=R_{2} \nabla_{1}^{2} W_{0}+\left(\overline{W_{0} T_{0}}-\overline{W_{0} T_{0}}\right) \nabla_{1}^{2} W_{0}$

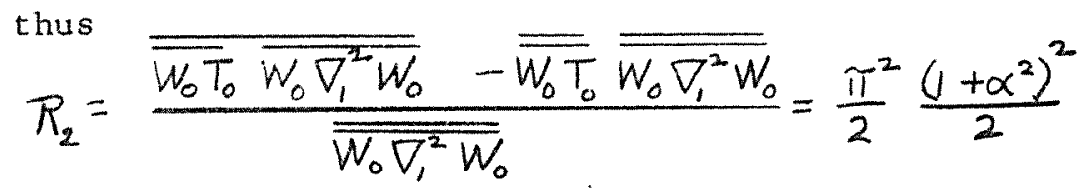

To this order: $R=R_{0}+\epsilon^{2} R_{2}$

or $\epsilon^{2}=\frac{2 \alpha^{2}}{\pi^{2}\left(1+\alpha^{2}\right)^{2}}\left(R-R_{0}\right)$ 


$$
\begin{gathered}
\overline{\overline{W T}}=\epsilon^{2} \overline{\overline{W_{0} T_{0}}}=2\left(R-T_{0}\right) \\
\overline{W T}=\epsilon^{2}{\overline{W_{0} T_{0}}}=4\left(R-R_{0}\right) \sin ^{2} \pi z
\end{gathered}
$$

and $\frac{\beta}{\beta_{m}}=1+2\left(\frac{R-R_{0}}{R}\right)\left[1-2 \sin ^{2} \pi z\right]=1+2\left(1-\frac{R_{0}}{R}\right) \cos 2 \pi$

and $\quad \frac{H}{K \beta_{m}}=1+\frac{2\left(R-R_{0}\right)}{R}$

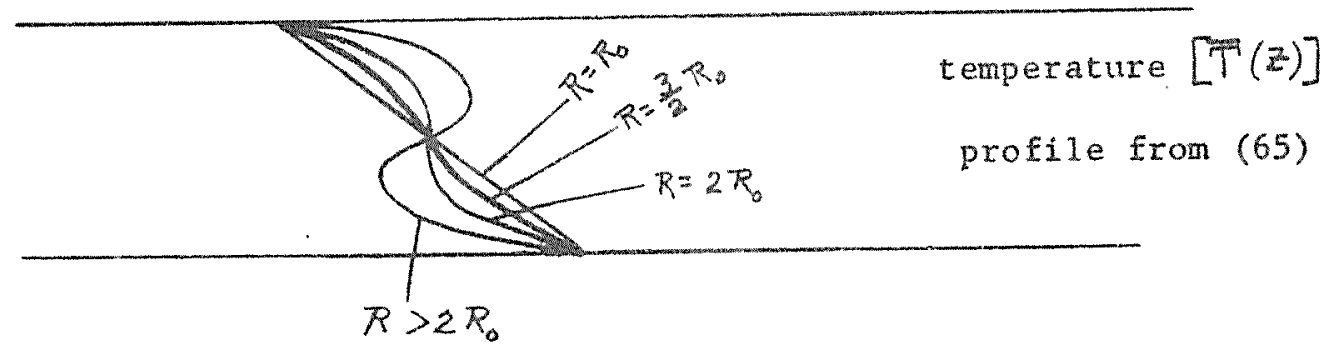

The $E^{2}$ result breaks down below $R=2 R_{c r i t}$ as may be seen by going to higher orders. The fact that the $\epsilon^{2}$ result gives a negative $\beta$ for $R>2 R_{\text {crit }}$ is not conclusive proof that it is incorrect there, but it does turn out that the temperature inversion disappears in higher approximations.

The $M-V$ paper calculates the heat transport to 6 th order:

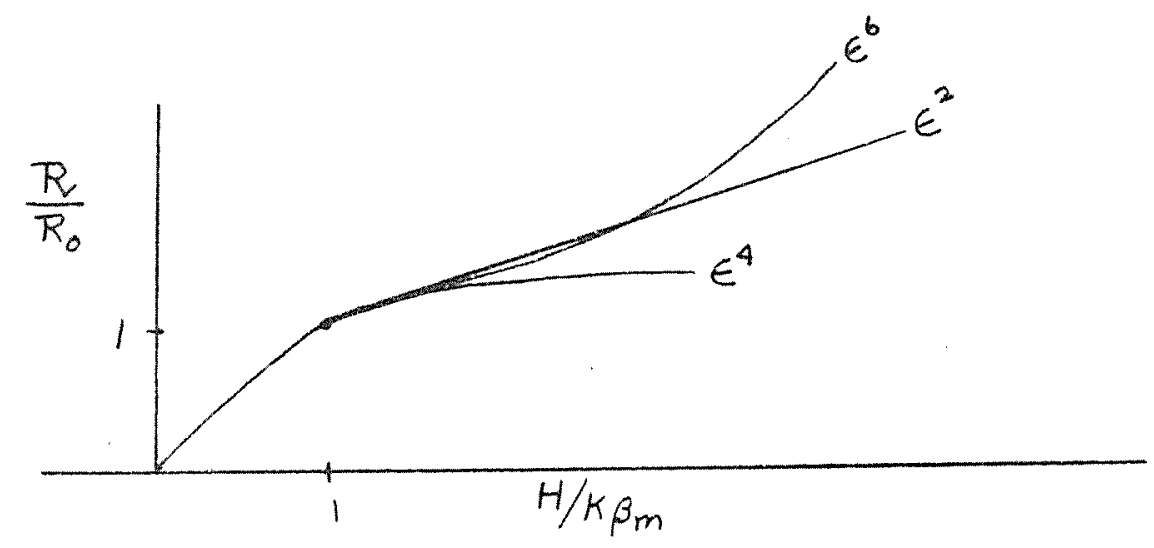


A value of 7 for $\sigma$ was used in this calcuiation (appropriate for $\mathrm{H}_{2} \mathrm{O}$ ).

Malkus and Veronis made calculations for other plan forms. The following graph shows the amount of convective heat transport in the neighborhood of $\mathrm{R}_{\text {crit }}$ for various rectangles. $\left(\epsilon^{2}\right.$ result).

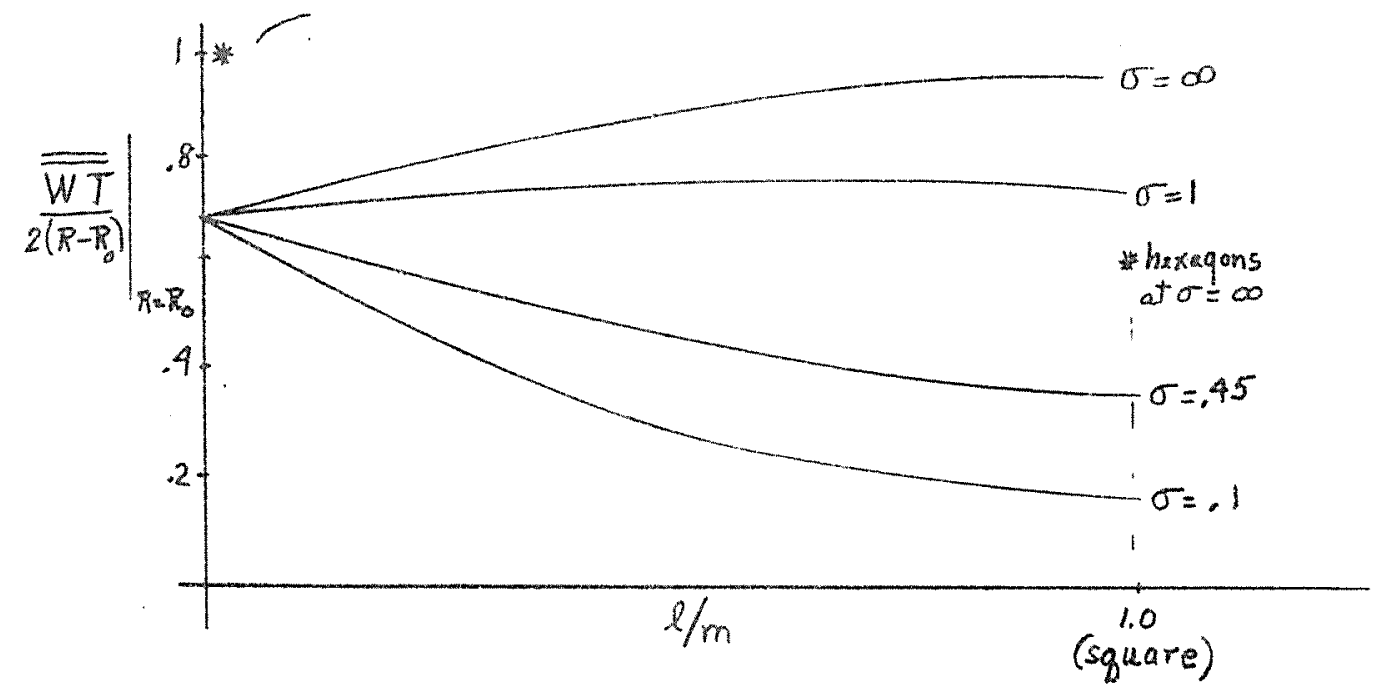

Note that the result for rolls differs from the result for rectangles in the limit $\frac{\text { length }}{\text { width }} \rightarrow \infty$.

VI A Relative Stablity Criterion

Now that we have found a wide variety of statistically steady motions that can exist in the convective region, we must determine which solution actually does exist. Each solution represents a different geometric flow pattern; each transports a different amount of heat. Which one does the fluid pick?

There are at least 3 ways we could approach the problem. 
a) We could hinge our expansion about $R>R_{c}$ and solve a time dependent initial value problem

b) (much simpler, but still much too difficult), we could investigate the stability of each finite amplitude solution, by applying arbitrary infinitesimal perturbations.

c) We can try to establish a criterion based entirely on the moments of the fields which will enable us to compare difo ferent maintained motions and decide which one is more likely to be stable. (the relative stability criterion).

$$
\text { Imagine that we have a super-critical state with a }
$$

maintained field

$$
\vec{u}(x, y, z) T-T_{m}=\bar{T}(z)+T(x, y, z)
$$

Then we add a disturbance

$$
\vec{u} \rightarrow \vec{u}+\vec{u}^{\prime} \quad \bar{T}+T \rightarrow \bar{T}+T+T^{\prime}
$$

then $\frac{\partial \vec{u}}{\partial t}+\vec{u}^{\prime} \cdot \vec{\nabla} \vec{u}+\vec{u} \cdot \vec{\nabla} \vec{u}^{\prime}+u^{\prime} \cdot \nabla u^{\prime}=-\frac{1}{\rho} \nabla p^{\prime}+\gamma T^{\prime} k^{2}+\nu \nabla^{2} \vec{u}^{\prime}$

$$
\begin{gathered}
\frac{\partial T^{\prime}}{\partial t^{\prime}}+\vec{u} \cdot \nabla T^{\prime}+u^{\prime} \cdot \nabla(\vec{T}+T)+u^{\prime} \cdot \nabla T^{\prime}=K \nabla^{2} T^{\prime} \\
\nabla \cdot \vec{u}^{\prime}=0
\end{gathered}
$$

The power integrals are then

$$
\begin{gathered}
\frac{1}{2} \frac{\partial}{\partial t} \overline{\overline{u^{\prime} \cdot \overrightarrow{u^{\prime}}}}+\overline{\overline{u^{\prime} \cdot\left(\vec{u}^{\prime} \cdot \vec{\nabla}\right) \vec{u}}}=\gamma \overline{\overline{W^{\prime} T^{\prime}}}+\overline{\overline{\nu \vec{u}^{\prime} \cdot \nabla^{2} \overrightarrow{u^{\prime}}}} \\
\frac{1}{2} \frac{\partial}{\partial t} \overline{\overline{T^{\prime 2}}}+\overline{\overline{T^{\prime} \overrightarrow{u^{\prime}} \cdot \vec{\nabla}(\bar{T}+T)}}=k \overline{\overline{T^{\prime} \nabla^{2} T^{\prime}}} \\
\overline{\overline{T^{\prime} u^{\prime} \cdot \nabla T}}-\overline{\overline{T^{\prime} W^{\prime} \beta}}
\end{gathered}
$$


Now suppose we know all the possible maintained motions. In order to examine their relative stability we consider only a special class of disturbances, namely, those which themselves have the form of some other solution of the basic equations.

i.e. assume that at $t=0$

$$
\vec{u}=A \vec{u}, \quad T=B T_{1}
$$

where $\vec{u}_{1}, T_{1}$ are possible maintained motions. In this situation the "triple correlations" $\overline{\overline{(\vec{u} \cdot \cdot(\vec{u}, \vec{\nabla}) \vec{u}})}$ and $\overline{\overline{T_{1} \vec{u}, \cdot \vec{\nabla} T}}$ vanish, in the 1st order finite amplitude treatment.

So:

$$
\begin{aligned}
& \frac{1}{2} \frac{\partial}{\partial t} \overline{\left(\overline{v^{\prime} \cdot v^{i}}\right)_{t=0}}=A^{2} v \overline{\overline{u_{1}} \cdot \nabla^{2} \overline{u_{1}}}+\gamma A B \overline{W_{1} T_{1}} \equiv-A^{2} I_{1}+A B I_{1} \\
& \frac{1}{2} \frac{\partial}{\partial t} \overline{\left(T^{\prime 2}\right)_{t=0}}=K B^{2} T_{1} \nabla^{2} T_{1}+A B\left(\overline{W_{1} T_{1} \beta}\right) \equiv-B^{2} I_{3}+A B I_{4}
\end{aligned}
$$

We assert that if the system $\vec{U}^{\prime}, T^{\prime}$ grows inftially, the underlying motion $\vec{u}$; $T$ will be unstable. Granting this assertion, then there will be instability provided we can find $A$ and $B$ so that

$$
\begin{aligned}
& -A^{2} I_{1}+A B I_{2}>0 \\
& -B^{2} I_{3}+A B I_{4}>0
\end{aligned}
$$

which in view of the fact that $I_{1}$ and $I_{3}$ are positive-definite amounts to the condition

$$
I_{2} I_{4}>I_{1} I_{3}
$$

for instability.

i.e. $\gamma \overline{\overline{W_{1} T_{1}}} \overline{\overline{W_{1} T_{1} \beta}}>k \overline{\overline{T_{1} \nabla^{2} T_{1}}} \nu \overline{\overline{u_{1} \cdot \nabla^{2} u_{1}}}$ 
But by assumption, the $\vec{u}_{1}, \vec{T}_{1}$ field satisfies the power integrals. So the condition for instability becomes

$$
\overline{\overline{W_{1} T_{1}}\left(\beta-\beta_{1}\right)}>0
$$

Using (12) in the previous section this becones

$$
\overline{\beta_{1}^{2}-\beta \beta_{1}}>0
$$

or $\bar{\beta}_{1}^{2}>\overline{\beta \beta_{i}}$ (bar represents $\mathrm{m}$-average here)

By virtue of Schwarz' inequality

$$
\overline{\beta^{2}} \overline{\beta_{1}^{2}} \geqslant \bar{\beta}^{2}
$$

A sufficient condition for instability is

$$
\overrightarrow{\beta_{1}^{2}}>\overline{\beta^{2}}
$$

Thus a necessary condition for stability is that the solution must have a larger value of $\bar{\beta}^{2}$ than any other solution, and along this line we may think of the "relative stability" of two solutions as being given by a comparison of the two values of $\overline{\beta^{2}}$. of course, we have restricted ourselves to first order in the finite amplitude expansion, by requiring that the triple correlations vanish. Indeed, in the first order finite amplitude treatment, maximizing $\overline{\beta^{2}}$ is equivalent to maximizing the heat flux, and we shall tentatively adopt the condition of ${ }^{\prime} H_{\max }$ "for determining the preferred state.

Reference: Malkus and Veronis (1958) J.Fluid Mech. 4, 225. 


\section{Relative Stability and Entropy Production}

We found that the relative stability criterion

$$
\left\{\overline{W T}\left(\beta_{0}-\beta\right)\right\}>0
$$

is equivalent either to maximising $\mathrm{H}$ for a given mean temperature gradient $\beta_{m}$ or minimising $\beta_{m}$ for a given heat transport $H$. We will explore the connection between the entropy prow duction in irreversible thernodynamic systems and the criterion of relative stability advocated earlier.

The 'local' themodynamic entropy of the system as a function of the temperature and denstty satisfies the equation

$$
T \frac{d 8}{d t}=\frac{d u}{d t}+p \frac{d}{d t}(1 / p)
$$

where $\frac{d}{d t}$ is the substantial derivative and $U$ the internal energy of the system. Within the framework of the Boussinesq approximation only the first term survives and is given by

$$
p \frac{d u}{d t}=k \nabla \cdot(\nabla T)
$$

Incorporating this expression for the rate of change of internal energy, the entropy equation becomes

$$
\rho T \frac{d s}{d t}=K \nabla \cdot(\nabla T)
$$

For a statistically steady state in a bounded region the entropy production in the interior will be balanced against the outflowing entropy flux at the boundaries such that 


$$
(\overline{\overline{\partial s}})=0=-\left(\overline{\left(\frac{\partial s}{\partial t}\right)}\right)_{B_{0}}+\left(\overline{\frac{\partial s}{\partial t}}\right)_{I_{n}}
$$

Equating the corresponding parts of Eqn. (21) which arise on integration, we get

$$
\begin{aligned}
& \overline{\left(\frac{\partial S}{\partial t}\right)_{B_{0}}}=\left(\frac{K \nabla T}{\rho T}\right)_{B_{0}} \\
& \overline{\left(\frac{\partial s}{\partial t}\right)_{l_{n}}}=\frac{k}{\rho}\left(\overline{\nabla T \cdot \nabla \frac{1}{T}}\right)
\end{aligned}
$$

which can be rewritten as

$$
\begin{aligned}
& \rho T_{m}^{2}\left(\overline{\frac{\partial s}{\partial t}}\right)_{B_{0}}=H, \beta_{m} \\
& \rho T_{m}^{2} \overline{\left(\frac{\partial s}{\partial t}\right)_{I_{n}}}=K \overline{\overline{\beta^{2}}}+K \overline{(\nabla T \cdot \nabla T)} \\
& =k \overline{\overline{\beta^{2}}}+(\overline{\overline{W T} \beta})
\end{aligned}
$$

Recalling our stability argument, Equation (19), in relation to this equation, we find that for a fixed flux $\mathrm{H}$, the stable solution is one of MINIMUM entropy production, while for a fixed $\beta_{m}$, (force field) the stable solution is one of maximum entropy production.

A comparison of this criterion with a theorem proved by Prigogine, (DeGroot, 1959), in irreversible thermodynamics is suggestive. It states: For a system with a variety of forces and fluxes, having a linear force-flux relationship between them, if we fix some of the forces on the system, the entropy production. will be minimum, when the remaining fluxes vanish. Vice versa, if we keep some fluxes on the system constant, entropy production is again minimum when the remaining forces vanish. This reciprocal 
relationship between forces and fluxes derives explicitly from their linear character. The forms which this theorem is likely to take, when one incorporates a non-linear relationship between forces and fluxes is far from clear.

The argument of the relative stability criterion as seen earlier, suggests that for a fixed flux, H, the stable solution is one with minimum entropy production, i.e. minimum $\beta_{m}$. The corresponding result for fixed $\beta_{m}$ is that the heat flux, $H$, be a minimum. Thus the principle of minimum entropy production can be expressed either as minimum $\beta_{m}$ for fixed $H$ or as maximum $H$ for fixed $\beta_{m}$. This divergence from the results of linear thermodynamical arguments suggests a motivation for the further study of this criterion.

\section{Turbulent Convection}

An alternate approach to the theory of turbulent convection, widely differing from the mechanistic approaches considered earlier has been put forward by Willem Malkus (1954, 1960). We will discuss now some of the motivations which go to form this venture. The experience gained from the study of finite amplitude perturbations of convection phenomena, suggests that among the various motions in a convective field, the most unstable one is the one which transports the maximum heat. And anticipating a proof of this criterion, we assume that the pattern of motions is the one which maximizes heat transport. 
We also further assume that there is a smallest scale of motion which contributes to the heat transport in the fully evolved systern.

In fintte amplitude studies the zero average non-linear terms, which are the fluctuating interactions of the temperature and velocity fields that do not contribute to the mean field, were found always to have a stabilizing influence. 'Stabilizing' in this context refers to their tendency to reduce the heat trans port. A1so the finite amplitude studies, even to higher orders in $\epsilon$, suggest that the mean proxile tends to be positive, all through the region. This tempts one to extend the results and knowledge gained from the linear and finite amplitude studies to a fully turbulent field. Following this, we assume that the smallest scale of motion whtch contributed to the heat transport Is the same as the smallest scale of motion which is marginally stable on the mean profile.

We will start with the equation

$$
\mu=K \beta+\overline{W T}
$$

or

$$
\frac{k \beta}{H}=\frac{(1-\overline{W T})}{H} \geq 0
$$

(we make use of the fact that $\beta$ never changes sign)

We can write

$$
\frac{K \beta}{H}=I^{2}
$$


with $I=\sum_{0}^{\infty} I_{n} \psi_{n}$

where the $\psi_{n}$ are some suitable set of orthogonal functions which satisfy the boundary and symetry conditions of the problem. From Eqns. $(10-13)$ above, it follows that

$$
\frac{\beta}{\beta_{m}}=1+\frac{(\overline{\overline{W T}})-(\overline{W T})}{K \beta_{m}}
$$

If we assume that there is a smallest scale of motion. and that this is the smallest contributor to the heat transport, we can truncate the series for $I$ with terms which correspond to this scale. Strictly such a procedure will be valid only if we krew the true eigenfunctions of the problem. But since we intend constructing a variational scheme, any function which satisfies the boundary conditions will give an upper bound to $H$. The true upper bound may be far below this. We can by minimising Equation (3) for a given $\eta_{0}$ find a relation between $H \beta_{m}$ and $\eta_{0}$. We can link the $\eta_{0}$ to the smallest scale unstable on the mean profile and consequent $1 y$ the Rayleigh number, through the second assertion.

\section{Free Boundary Condition Solutions.}

The appropriate set $\psi_{n}$ for the case of the free boundary conditions are the simple trigonometric functions

$$
\psi_{n}=\cos 2 n \phi: \psi_{0}=1 \quad 0 \leq \phi \leq \pi
$$

In order that $\psi_{\eta_{0}}$ correspond to the scale $\eta_{0}$, the sum for I is terminated at $\frac{n_{0}}{2}$.

Now

$$
\begin{aligned}
\frac{K \beta_{m}}{H} & =\frac{1}{\pi} \int_{0}^{\pi} \frac{K \beta}{H} d \phi \\
& =\frac{1}{2} \sum_{0}^{n_{0} / 2} I_{n}^{2}\left(1+\delta_{n, 0}\right)
\end{aligned}
$$


Also since $\begin{aligned} \beta(0)= & \beta(\pi)=\frac{H}{k} \\ & \left(\sum_{n=0}^{n_{0} / 2} I_{n}\right)^{2}=1\end{aligned}$

To maximise $\mathrm{H}$, we 11 minimise Equation (7), subject to the constraints (8), using Lagrange's undetermined multipliers, to get

$$
\frac{\partial}{\partial I_{n}}\left(\frac{\beta_{m} K}{H}\right)=0 \text { when } I_{n}=\frac{2}{n_{0}+1} \text { and } I_{0}=\frac{1}{n_{0}+1} \cdots
$$

In terms of these $I^{\prime} s$

$$
\begin{aligned}
\frac{K / \beta_{m}}{H} & =\frac{1}{2} \sum_{0}^{n_{0 / 2}} I_{n}^{2}\left(1+\delta_{n_{10}}\right) \\
& =1 /\left(n_{0}+1\right)
\end{aligned}
$$

or

$$
H=k \beta_{m}\left(n_{0}+1\right)
$$

And the expression for $I$ becomes

$$
\begin{aligned}
I & =\sum_{j}^{n_{0} / 2} I_{n} \psi_{n}=\frac{2}{n_{0}+1} \sum_{1}^{n_{0} / 2} \psi_{n}-\frac{1}{n_{0}+1} \\
& =\frac{2}{n_{0}+1} \sum_{1}^{n_{0} / 2} \cos n \phi-\frac{1}{n_{0}+1} \\
& =\frac{1}{n_{0}+1}\left\{1+\frac{\sin \left(n_{0}+1\right) \phi}{\sin \phi}\right\}-\frac{1}{n_{0}+1} \\
I & =\frac{\sin \left(n_{0}+1\right) \phi}{\left(n_{0}+1\right) \sin \phi} \\
\text { or } \operatorname{recal1} & \text { that } \\
& \frac{K \beta}{K}=I^{2}=\frac{\sin ^{2}\left(n_{0}+1\right) \phi}{\left(n_{0}+1\right)^{2} \sin ^{2} \phi} \\
\frac{\beta}{\beta_{m}} & =\frac{\sin ^{2}\left(n_{0}+1\right) \phi}{\left(n_{0}+1\right) \sin ^{2} \phi}
\end{aligned}
$$


These relations between $K$ and $\left(\eta_{0}+1\right)$ and $\beta$ and $\beta_{m}$ are independent of any assumptions on the dynamics of the flow. It assumes only that there exist a smallest scale of motion contributing to heat transport and that the temperature profile $\beta$ remain positive everywhere.

If we assume that $\eta_{0}>>1$ we can integrate equation (12), to get the temperature distribution

$$
\begin{gathered}
\bar{T}(\phi)-T_{m}=\frac{\Delta T}{2 \pi} \frac{1}{n_{0}+1}\left\{\operatorname{ctn} \phi, o\left(\frac{1}{n_{0}}\right)\right\} \\
\text { for } \phi>\frac{\pi}{n_{0}}
\end{gathered}
$$

The integral for the mean field for $\phi \simeq \frac{\pi}{n_{0}}$ and large $n_{0}$ is

$$
\begin{aligned}
\bar{T}(\xi) & -T_{m}=\frac{\Delta T}{2}-\frac{2}{\pi} \int_{0}^{\phi} \beta d \phi \\
& \simeq \frac{\Delta T}{2}-\frac{1}{n_{0}+1} \frac{H}{K} \frac{2}{\pi} \int_{0}^{\zeta} \frac{\sin ^{2} \xi}{\xi^{2}} d \xi \\
\bar{T}(\zeta)-T_{m} & \simeq \frac{\Delta T}{2}\left[1-\frac{2}{\pi} \operatorname{Si}(2 \xi)-\frac{\left.\sin ^{2} \xi\right]}{\zeta}\right]
\end{aligned}
$$

where $\zeta=\left(n_{0}+1\right) \phi$ and $\delta_{i}(x)$ is the sine integral.

We will now carry through our second assertion that this minimum scale which contributes to the heat transport is the same one which is critically stable on the mean field. With this assumption we can relate the $n_{0}$ to the Rayleigh number and thus end up with a quantitative relationship between $H$ and the Rayleigh number.

The equations for the disturbance $W^{\prime} T^{\prime}$ of the velocity and temperature fields which are marginally stable on the mean 
field are

$$
\begin{gathered}
\left(\frac{\partial}{\partial t}-k \nabla^{2}\right) T^{\prime}=\omega^{\prime} \beta \\
\left(\frac{\partial}{\partial t}-\nu \nabla^{2}\right) \omega^{\prime}=-\nabla p+\gamma T^{\prime} k \\
\nabla \cdot \varphi^{\prime}=0
\end{gathered}
$$

Eliminating the $T^{\prime}$ terms in the usual way, we get a single equation for $\omega^{\prime}$ as

$$
\left(\frac{\partial}{\partial t}-k \nabla^{2}\right)\left(\frac{\partial}{\partial t}-\nu \nabla^{2}\right) \nabla^{2} \omega^{\prime}=-\gamma \beta \nabla_{1}^{2} \omega^{\prime}
$$

where $\nabla_{1}^{2}$ is the horizontal Laplacian.

For free boundaries one can show that $\frac{\partial}{\partial t}=0$ for marginal stability whatever the form of $\beta$. Assuming a horizontal cellular motion with a wave number $\alpha$ we can write the equation for the disturbance in the marginally stable state in the form

$$
\left(\frac{\partial}{\partial \phi^{2}}-\alpha^{2}\right) \omega^{\prime}=-\alpha^{2} \frac{R}{\pi^{4}} \frac{\beta}{\beta_{m}} \omega^{\prime}
$$

We seek solutions of this equation which are extremal for $R$ and which satisfy the boundary conditions $\omega^{\prime}=0, \frac{\partial^{2} w}{\partial z^{2}}=0, \frac{\partial^{4} w}{\partial z^{4}}=0$ at $\phi=0$ and $\pi /\left(n_{0}+1\right)$. First we construct a positive definite variational form for $R$. Multiplying Equation (19) by $W^{\prime}$ and integrating over the domain of $\phi$

$$
\int_{0}^{\pi} \omega^{\prime}\left(\frac{\partial^{2}}{\partial \phi^{2}}-\alpha^{2}\right)^{3} \omega^{\prime} \alpha \phi=-\frac{\alpha^{2} R}{\pi^{4}} \int_{0}^{\pi} \frac{\beta}{\beta_{m}} \omega^{2} d \phi
$$


Substituting for $\left(\beta / \beta_{m}\right.$ from equation (12), we get

$$
R=-\frac{1}{\alpha^{2}} \frac{\int_{0}^{\pi} \omega^{\prime}\left(\frac{\partial^{2}}{\partial \phi^{2}}-\alpha^{2}\right)^{3} \omega^{\prime} d \phi}{\int_{0}^{\pi} \frac{\sin ^{2}\left(n_{0}+1\right) \phi}{\left(n_{0}+1\right) \sin ^{2} \phi} \omega^{\prime 2} d \phi}
$$

If $\left(n_{0}+1\right)$ is large compared to unity, the gradients

are large only near the boundary. An upper bound for $R$ is

then found from the integration over the first cycle $0-\frac{\pi}{\left(n_{0}+1\right)}$.

It will be convenient to work with the new variable $\xi=\left(n_{0}+1\right) \phi$

and for this limit of large $\left(n_{0}+1\right)$, we rewrite equation

(21), as

$$
\frac{R}{\left(n_{0}+1\right)^{3}}=-\frac{1}{\alpha^{2}} \frac{\int_{0}^{\pi} \omega^{\prime}\left(\frac{\partial^{2}}{\partial \zeta^{2}}-\alpha^{2}\right)^{3} \omega^{\prime} d \zeta}{\int_{0}^{\pi} \frac{\sin ^{2} \zeta}{\zeta} \omega^{2} d \zeta}
$$

The simplest trial function satisfying the boundary

conditions is $\sin \zeta$. Minimising (21') for $R$ using this trial function we get

$$
\frac{R}{\left(n_{0}+1\right)^{3}}=R_{C F}=1533
$$

We can carry through the same analysis for the nottoo-large $n_{0}$ case, where the disturbances are not restricted to a region of order $\eta_{0}^{-1}$ near the boundary but penetrate throughout the body of the fluid. $R$ is proportional in this case to the fourth power of $\left(n_{0}+1\right)$. The actual constant of proportion ality in this case may well be very sensitive to the choice of the trial function. But this suggests a different heat transport- 
Rayleigh number relationship, for low Rayleigh numbers.

For the boundary function case

$$
H=K \beta_{m}\left(\frac{R}{R_{C F}}\right)^{1 / 3} \quad R_{C F}=1533
$$

For the body function case

$$
H=K \beta_{m}\left(\frac{R}{R_{B F}}\right)^{1 / 4} \quad R_{B F}=656
$$

The $R^{1 / 3}$ law for the heat transport has been predicted by Priestly (1954) using a dimensional similarity argurent. As a qualitative approach, it could not predict the constant of proportionality $R_{C F}$.

\section{Rigid Boundary Condition Solutions}

This case is more difficult to deal with, since the $\psi_{n}$ 's which satisfy the rigid boundary conditions are more difficult to deal with than the free boundary ones. The studies of Pellew and Southwell (1940) give for the critical Rayleigh numbers in the free-free anu rigid-free cases, 656 and 1100 respectively. This means that the temperature difference which sustains a marginal disturbance, in the rigiu-free case, is $\left(\frac{110 c}{65 i}\right)^{\frac{1}{4}}$ times that necessary to sustain a similar marginally stabie free-free disturbance.

If we assume that this ratio remains the same for the critical Rayleigh number of the smallest marginally stable motion even in the case of turbulent fields, the boundary region will be 
extended until

$$
\frac{\Delta T_{R}}{\Delta T_{F}}=\left(\frac{R_{F R}}{R_{F F}}\right)^{1 / 4}=\left(\frac{1100}{656}\right)^{4}=1.1382
$$

The corresponding heat flux would be given by Equation (23) with $R_{C F}$ replaced by $R_{C R}=2533$.

\section{Critical Remarks and Comparisons with Other Theories}

As has been pointed out earlier, the question of getting a relation between $H$ and $\eta_{0}$ depends only on three assertions: 1) The heat transport tends to be a maximum 2) There exists a smallest scale which contributes to the heat transport and 3) The temperature gradient remains positive everywhere.

The first assertion is debatable. It is suggested by the results of the linear as well as the finite amplitude theory. The second assertion depends very critically on whether a tail for the spectrum exists or not. This should be established by a more detailed mechanistic study of tue non-linear transfer process. The third assertion is also suggested by finite amplitude studies and, though borne out by experiment, most probably is a subtle aspect of the inertial terms.

The second section of the theory asserts a connection between the $n_{0}$ of the heat transport and the Rayleigh number, through a variational analysis of the disturbance equations in the marginally stable state. A defense of this assertion will 
require the development of a quantitative theory of the transfer process.

The only other theory of turbulent transport is the one advocated by Priestly $(1954,1955,1956)$. It is based on arguments of similarity. It asserts that the effects of conduction and viscous dissipation should be restricted to boundary layers. It draws its analogy and support from the successes of similarity theory in the fully-developed turbulent fiow in channels. As is unavoidable in any such theories, it has disposable con srants. Though it also predicts a qualitative $R^{1 / 3}$ law for the heat transport, the temperature profile is predicted as

$$
\log \left(\frac{T}{T_{a}}\right) \approx 2^{-1 / 3}
$$

The current theory for the same region makes quantitative predictions

$$
\begin{gathered}
\log \frac{T}{T_{a}}=c \theta_{0} \frac{z_{0}}{2} \\
\text { with a value for } C=2.0
\end{gathered}
$$

\section{Comparison with Experiments}

The experiments of Townsend and Thomas (Thomas \& Townsend, 1957; Townsend, 1958) have been used to test the quantitative predictions of the theory. The experiments, however involved large temperature differences and thene is a difficulty in connecting the equivalent mean temperature for the convection process and the

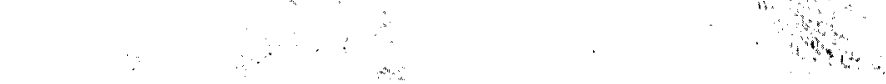
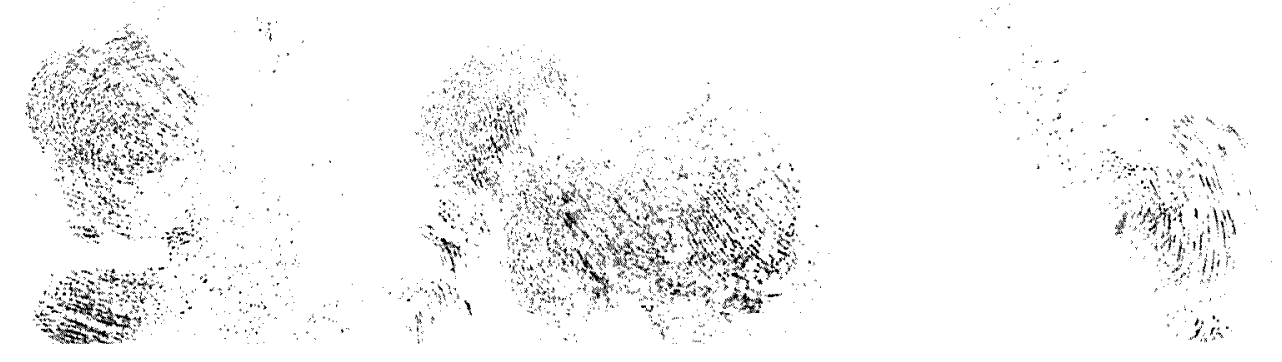
observed ambient temperature. Despite this, the experimental value of $R_{F}=2000 \pm 200$ corresponds to a heat transport only eight per cent more than the predictions of the iheory. A more significant test for the theory will be the comparison of the temperature profile, where the two theories predict a widely different character. The experiments of Townsend confirm the qualitative predictions of Malkus, while the constants predicted and measured differ within $30 \%$

It should be possible to determine the range of validity of the theory and improve it by designing experiments which are more directly intended to lest its predictions. On the other hand, improving the trial functions in the variational scheme of the theory will limit the errors introduced there. 


\section{References}

DeGroot (1959) Thermodynamics of Irreversible systems. North-Holland Publishing Co., Amsterdam.

W. Malkus (1954) Proc. Roy. Soc. A, 225, 196.

(1960) J. Heat Transport (in press).

D.B.Thomas and A.A.Townsend (1957)

J.F1. Mech. 2, 473.

A.A. Townsend (1958)

J.F1.Mech. 5, 209.

A.Pellew and R.V.Southwel1 (1940)

Proc.Roy.Soc. A, 176, 312.

Priestly (1954) Aust. J. Phys, I, 176.

(1955) J.Roy.Met.Soc. 81, 139.

(1956) Proc.Roy.Soc. A, 238, 287. 


\section{The Wind-Driven Ocean Circulation}

\section{The observational basis*}

\section{Introduction}

All descriptions of reality are simplified: in order to present a coherent picture of observations we really need a theory to act as a framework. There is as yet no theory of oceanic circulation which could form a framework for a discussion such as the present one. We are concerned with varying time and length scales, with surface currents, with integrated currents, and with transports: those observations which are significant depend on the theoretical background. The theory which is most developed today is that of the mean circulation. Therefore, the features of the oceanic circulation which will be discussed here will be those which emerge from averages of observations over long periods, so as to smooth out seasonal fluctuations, where possible. There are two main sources of information about the ocean currents:

(a) Many years' reports of surface currents, as observed by merchant and naval vessels, have been combined by the hydrographic offices of different nations in the form of charts of surface currents. One of the best of these is due to Schott (1942). These charts are based on enough individual observations to give some significance to the means. *Sections (1) and (2) are notes from a lecture by H. Stommel. 
(b) Mean subsurface currents are not measured directly, but Inferred from the observed mean distribution of density in the oceans. Seasonal changes in density are largely confined to the upper 200 meters of the ocean, so that for veeper layers, the mean density field is found to a first approximation from individual observations.

2. Geostrophic Currents Associated with Mean Density Field. The deep waters of the ocean are cold and of remarkably uniform density. Thus below 3000 meters depth, the horizontal variations in density are less than $0.01 \%$. Radio carbon ages of 1200 years, observed in deep water suggest that the mean currents in these deep waters are also small - with mean velocities less than 1 millimeter a second (although turbulent fluctuating velocities of one hundred times that amplitude are common - as observed by Swallow's pingers).

In the surface layers, exposed to heating in the tropics, and cooling near the poles, there are large differencas in density from place to place - of as much as $0.5 \%$. In the absence of constraints (which in the ocean are due to rotation of the earth), the low density surface waters near the equator would spread out toward the poles.

In the atmosphere it has been found, by a comparison of observed winds aloft, and the pressure field, that large scale 
winds are geostrophic. We expect to find that the mean ocean currents are geostrophic also.

Pressures cannot be systematically and easily measured in the oceans, but if we could do this we would expect that some sort of pressure patterns, related to the flow, would be found. We would like then to be able to deduce pressures from other data. The technique used is to integrate the hydrostatic equation from some depth up to the surface, at a given location, using the observed values of temperature and salinity to give the lensity along the vertical colum of water. The ifficulty here is th.t we do not know the pressure at the bottom of our column, even if this extends to the bottom of the ocean. We introduce the assumption that below some depth, as yet unspecified, negligible pressure gradients exist. We will discuss this in more detail a little later on, but if this is granted, different columns of water, with different density structure in the vertical, will h ve different pressures at the same depth; that is to say, by combining results from many stations, we can compute pressure gradients at various levels. An equivalent :ay of presenting such data is to compute the height of water at a given place, above the basic level of no pressure gradient, needed to reach a certain pressure. Areas of low(high) pressure then become areas of low (high) height. 
for the surface of the ocean, (with a constant height subtracted from all readings), so that the map can be thought of as showing the undulations of the sea surface.* The geostrophic curtents would then be along these height contours with speeds inversely proportional to the contour spacing. A study of the map shows areas of high and low height, areas of concentrated contour lines (strong currents), and could be thought to give a fair picture of mean circulation. However, we cannot demonstrate by direct current measurements that the flow actually is geostrophic, as assumed, and, more serious, we are in doubt as to how representative this map is of the "pressure" pattern. Thus, if a coarser contour interval than the $20 \mathrm{~cm}$ interval used were chosen, very much detail would be lost, while a finer contour interval would result in an extremely complex pattern. Would efther of these be as typtcal of the ocean as the map drawn?

We will therefore pass on to a consideration of the factors which make a map like this of somewhat uncertain significance. These are:

(i) choice of reference level (of no horizontal pressure gradient);

(ii) effect of spatial variations of atmospheric pressure;

(iii) seasonal effects;

(iv) short period fluctuations.

\footnotetext{
* The height interval between contours is $20 \mathrm{~cm}$.
} 
We will discuss each of these factors in turn.

(i) If we consider two different vertical columns of sea water, say in the high near Japan and near Antarctica, we might observe the following temperature structure:

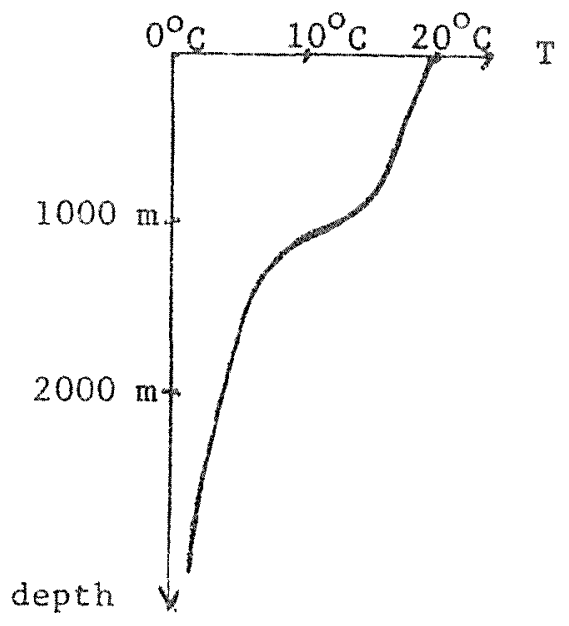

near Japan

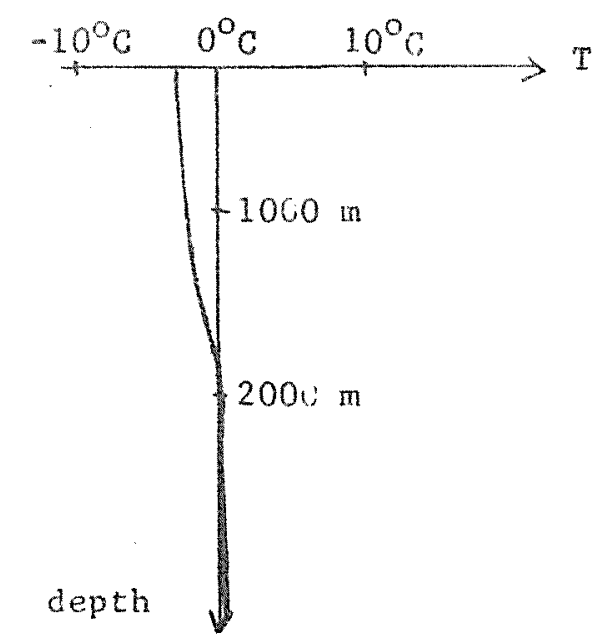

Antarctica

In the surface layers the water off Japan is much warmer than the water off Antarctica, hence the former is less dense than the latter. (The former is also more saline which will offset the density difference somewhat.) Tre main point, however, is that both columns reach more or less the same properties at some great depth; thus, if we started integrating the hydrostatic equation from the bottom of the ocean in each case we would not have a horizontal pressure difference between the two stations until we reached a level at which the properties of the two columns began to differ. We would then have to go through a 
longer column in the water off Japan to reach atmospheric pressure than in the water off Antarctica, assuming the same atmospheric pressure at both stations. We thus see that the map reflects the density distribution in the upper layers of the sea. Further, we see some basis for the assumption of no horizontal pressure gradients at some large depth. We would like to know the effect that various choices of the depth of no horizontal pressure giadient have on our "pressure" map. In principle we would like to choose various such depths and actually determine this effect, but paucity of deep observations* precludes this. (There are only about 1000 observations in the last 40 years which have gone down to 4000 meters.) What we can do is choose a few good selected stations and calculate pressure differences between them using various depths of no horizontal pressure gradient. We find that the map presented in class will be essentially independent of this reference level so long as it is at or below 1000 meters. (But: the Antarctic circumpolar current has large density differences to great depths).

(ii) As indicated in the discussion (i) above, the spatial variation of atmospheric pressure should actually be taken into account in the computations, and this will shift the contours slightly.

\section{(iii) Seasonal effects give variations in height of about}


$\pm 6 \mathrm{~cm}$., which is well within the $20 \mathrm{~cm}$ contour interval chosen, so that their effect should not alter this particular map too much. (iv) Short period fluctuations may give variations in helght of about $\pm 4 \mathrm{~cm}$., so we may say the same about them as about seam sonal effects. This is assoctated with short term movements up and down, by about 50 meters, of the isothermal surfaces. W. thus conclude that the map is to a good extent representative, and have defined to some extent the range of variation to be expected.

General Referenc:s:

Schott, G., "Die Grundlagen einer Weltkarte der Meeresstromungen", Ann. der Hydrographie und Maritimen Meteorologie. 70 (1942): 329-340.

Stonmel, H., "The Gulf Stream", U.Cal.Press, (1958).

Sverdrup, H.U., M.W.Johnson, and R.H.Fleming, "The Oceans", Prentice-Hall (1942). 


\section{Equations for Oceanic Motions}

We have had an introduction to the study of ocean circulation in the last lecture, by $H$. Stommel. We saw that there were many features to explain, and many gaps in our theoretical and descriptive knowledge. However, certain large-scale features seem to be "quasi-steady", for example the Gulf stream: this current changes location, and has large eddies associated with it, but in a statistical sense it is a steady feature of the oceanic circulation.

We would like to be able to discuss theoretically the occurrence of broad eastern currents, of sharp, fast western currents, and the separation of the latter from the coast. We will orient ourselves to this problem, and as a first step we will study the " $\not$-plane" approximation to the equations of motion.

\section{The $\beta$-Plane Approximation}

The dynamics of the oceans are properly described by the equations of motion for a fluid held by gravity to a rotating, spherical globe. Spherical coordinates are thus naturally indicated. (We will jgnore the actual elliptical, or possibly pear, shape of the earth). Now, the depths of the oceans, and lateral scales of observed patterns, are less than the earth's radius. Thus if we assign a vertical scale D (depth), a lateral scale $L$, and denote by $R$ the earth's radius, we have $D \ll R$. Further, in genera1, $\mathrm{D}<<\mathrm{L}$. We can use these latter facts to 
reduce the basic equations in spherical coordinates to the form of the equations for fluid motion on a rotating "plane", a great simplification. The equations resulting from this reduction however, differ in a very essential respect from those which actually apply to motions relative to a rotating plane: they differ in that (due to the variation of the Coriolis paraneter), $E=2 \Omega \sin \phi$, with latitude in the original equations in spherical coordinates, the coriolis parameter in the final equations varies across the "plane" in a north-south direction. If the motion were referred originally to a rotating plane the Coriolis parameter would be simply a constant, f $\propto 2 \Omega$. The north-south distance in the "plane" is denoted by $y$, and $\mathrm{df} / \mathrm{dy}=\beta \neq 0$. Thus the motion must be thought of as occurring on an imagined rotating "plane" with this property. This "plane" is called the $\beta$-plane, the name being a result of the notation which sets $\beta=\mathrm{d} f / d y$, and the series of restrictions which lead to the final equations is called the $\beta$-plane approximation. We will now detail the steps in this reduction. The equations of fluid mechanics referred to a rotating globe are, in spherical coordinates, (see diagram): 


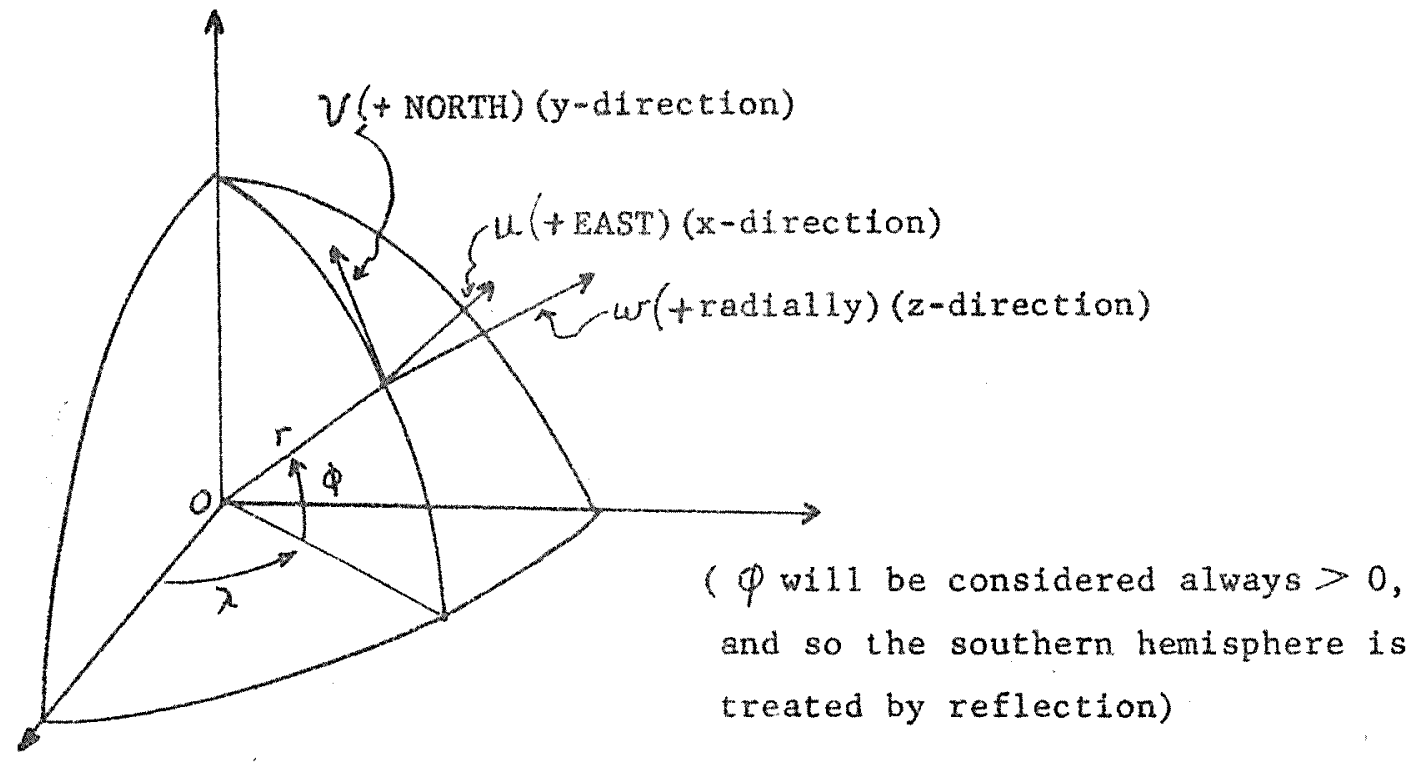

$$
\begin{aligned}
& \frac{\partial u}{\partial t}+w \frac{\partial u}{\partial r}+\frac{v}{r} \frac{\partial u}{\partial \phi}+\frac{u}{r \cos \phi} \frac{\partial u}{\partial \lambda}+\frac{u r u}{r}-\frac{u v \tan \phi}{r} \\
& +2 \Omega w \cos \phi-2 \Omega v \sin \phi=-\frac{1}{p r \cos \phi} \frac{\partial p}{\partial \lambda}+\text { frictional foces (1) } \\
& \frac{\partial v}{\partial t}+w \frac{\partial v}{\partial r}+\frac{v}{r} \frac{\partial v}{\partial \phi}+\frac{u}{r \cos \phi} \frac{\partial v}{\partial \lambda}+\frac{w v}{r}+\frac{u^{2} \tan \phi}{r} \\
& +2 \Omega u \sin \phi+4 \Omega^{2} r \sin \phi \cos \phi=-\frac{1}{\rho r} \frac{\partial p}{\partial \phi}+\text { frictional forces (2) } \\
& \frac{\partial w}{\partial t}+w \frac{\partial w}{\partial r}+\frac{v}{r} \frac{\partial w}{\partial \phi}+\frac{u}{r \cos \phi} \frac{\partial w}{\partial \lambda}-\frac{u^{2}+v^{2}}{r} \\
& -2 u \Omega \cos \phi-4 \Omega^{2} r \cos ^{2} \phi=-\frac{1}{\rho} \frac{\partial p}{\partial r}-g \text { frictional forces (3) } \\
& \frac{\partial w}{\partial r}+\frac{1}{r} \frac{\partial v}{\partial \phi}+\frac{1}{r \cos \phi} \frac{\partial u}{\partial \lambda}+\frac{2 w}{r}-\frac{v \tan \phi}{r}=-\frac{\vec{v}}{\rho} \cdot \nabla \rho .
\end{aligned}
$$


A few comments on the above equations will now be made.

The operator

$$
\frac{\partial}{\partial t}+\frac{\omega \partial}{\partial r}+\frac{v}{r} \frac{\partial}{\partial \phi}+\frac{\omega}{r \cos \phi} \frac{\partial}{\partial \lambda}=\left(\frac{D}{D t}\right)_{s}
$$

is common to the equations of motion. In the neighbourhood of any point this operator is merely the familiar operator

$$
\frac{\partial}{\partial t}+\frac{\omega \partial}{\partial z}+v \frac{\partial}{\partial y}+u \frac{\partial}{\partial x}=\left(\frac{D}{D t}\right)_{r}
$$

where $x, y, z$ are defined by the differential relations,

$$
d x=r \cos \phi d \lambda, d y=r d \varphi, d z=d r, \text { and are the spherical arc- }
$$

length and vertical height differentials. The fact that we

cannot make the above identification for other than differentials of $x, y, z$ reflects the divergence or convergence of the coordinate lines of the spherical coordinate system in the radial and $\phi$ directions. It is immediately seen that if the ranges of $r$ and $\phi$ are restricted sufficiently, we may be able to ignore the effects of radial and north-south divergence of the spherical coordinate lines, and make the identification $\left(\frac{D}{D t}\right)_{s}=\left(\frac{D}{D t}\right)_{r}$ The other terms in the equations of motion, except the pressure gradient and frictional terms, do not involve derivatives. These are Coriolis and centripetal accelerations due to the rotating reference frame, and centripetal accelerations due to the curvature of the spherical coordinate 1ines. We w111 now proceed to consider the simplification of these equations, considering first the vertical motions.

In the equation for vertical motions, (3), at least in so far as larger scale motions are concerned, all terms are assumed 
negligible except those expressing hydrostatic balance:

$$
-\frac{1}{p} \frac{\partial p}{\partial r}-g=0
$$

(It is customary to define the vertical so that $g$ includes the terms $4 \Omega^{2} r \cos ^{2} \phi$ and $4 \Omega$ rsin $\phi \cos \phi$ from the $u r$ and $v$ equations respectively: this redefines gravity as the sum of gravitation and centrifugal force due to the earth's rotation). This is reasonable as far as the terms $\frac{u^{2}+v^{2}}{r}$ and $2 u \Omega \cos \phi$ in the $w$ equation are concerned, since the redefined gravity force is always yery much larger than either of these. The same will be true of the terms in $\left(\frac{D}{D t}\right)_{S}$, for large scale motions, and of the frictional terms. Thus, assuming hydrostatic equilibrium does not impose any stringent conditions on our allowed speeds and scales of motion, due to the very large value of $g^{*}$ We will now consider the $u$ and $v$ equations of motion, (1) and (2).

It will be shown that it is possible to make a consistent scheme of restrictions which allows us to neglect all terms in the equations of motion which do not involve derivatives, except for certain desired Coriolis terms, and which also allows us to use the rectangular coordinates $x, y, z$ over a finite region. The terms

\footnotetext{
* We can now carry out a Boussinesq type approximation, so that pressure in the foregoing equations can be regarded as deviation from the adiabatic gradient, and density is some mean density for the layer (that is, for the ocean). In writing the continulty equation we then put $\nabla \rho=0$.
} 
neglected must be shown to be small compared to terms involving derivatives. To obtain estinates of orders of magnitude of derivatives we assume $\Delta u \sim u, \Delta v \sim v, \Delta w \sim w$, and, putting $\Delta r \sim D$, $\Delta y \sim \Delta x \sim L$, where $D, I$ are vertical and laceral scales respectively, will assume $\mathrm{D}<<\mathrm{L}, \mathrm{D}<<\mathrm{R}$. This is in accordance with the introductory discussion, and implies $r \sim R$. It will also be assumed that $u / v$ and $\frac{u}{u} \sim \frac{D}{L}$, and that $\tan \varphi \leqslant 1$. We will. now look at the individual terms in the $u, v$ equations, (1) and (2).

$$
\begin{aligned}
& \text { We will show that } \\
& \qquad w \frac{\partial u}{\partial r} \gg \frac{u r u}{r}, o r \frac{\partial u}{\partial r} \gg \frac{u}{r}
\end{aligned}
$$

in the $u$ equation. This is so, for,

$$
\frac{\partial u}{\partial r} \sim \frac{\Delta u}{\Delta r} \sim \frac{u}{D} \text {, and } \frac{u}{r} \sim \frac{u}{P+D} \sim \frac{u}{R}
$$

and, since $D \ll R$, the result is demonstrated. In order to show that

$$
\frac{1}{r} \frac{\partial u}{\partial \varphi} \gg \frac{u}{r} \tan \phi
$$

in the $u$-equation we note that

$$
\frac{1}{r} \frac{\partial u}{\partial \varphi} \sim \frac{\Delta u}{R \Delta \varphi} \sim \frac{u}{\Delta y} \sim \frac{u}{L}, \text { and } \frac{u}{r} \tan \varphi \sim \frac{u}{R} \cdot 1 \text {, }
$$

Hence, it is necessary to assume that $L \ll R$. In a precisely similar way $u \frac{\partial v}{\partial r} \gg \frac{\omega v}{r}$, and $\frac{v}{r} \frac{\partial v}{\partial \phi} \gg \frac{u^{2}}{r} \tan \varphi$ in the $v$-equation. Turning to the equation of continuity, (4), we will show that

$$
\frac{\partial w}{\partial r}-\frac{1}{r} \frac{\partial v}{\partial \varphi},
$$


so that three-dimensional divergence is retained as a possibllty. This is so, for

$$
\frac{\partial w}{\partial r} \sim \frac{\Delta w}{\Delta r} \sim \frac{w}{D} \text {, and } \frac{1}{r} \frac{\partial r}{\partial \theta} \sim \frac{1}{R \theta} \sim \frac{\Delta v}{\Delta y} \sim \frac{v}{L} \text {, }
$$

and since $w / / \sim D / L$, the result is denonstrated. We will show that

$$
\frac{1}{r} \frac{\partial u}{\partial \phi}>>\frac{2 w}{r}
$$

in the equation of continutity. This is so, for, $\frac{1}{r} \frac{\partial r}{\partial \phi} \sim \frac{V}{L}$, and $\frac{2 w}{r} \sim \frac{w}{R}$, and $\frac{v}{w} \sim \frac{L}{D} \gg \frac{L}{R}$, which demonstrates the result.

$$
\begin{aligned}
& \text { We will show that } \\
& \qquad \frac{1}{r} \frac{\partial v}{\partial \phi}>\frac{v}{r} \tan \phi .
\end{aligned}
$$

in the equation of continuity, for

$$
\frac{1}{r} \frac{\partial v}{\partial \phi} \sim \frac{v}{L} \text {, and } \frac{v}{r} \tan \phi \sim \frac{U}{R} \cdot 1 \text {, and }
$$

since $L \ll R$, the result is demonstrated,

Finally, consider the Coriolis terms. We do not wish to eliminate these completely, since we wish to preserve the effect of the rotating reference frame. However, in order to show that

$$
2 \Omega v \sin \phi>>2 \Omega u \cos \phi
$$

in the u-equation we observe that

$$
\frac{2 \Omega v \sin \phi}{2 \Omega w \cos \phi} \sim \frac{v}{w} \tan \phi \sim \frac{v}{w} \cdot 1 \sim \frac{L}{D} \gg 1 .
$$

The conditions we have laid down enable us to estimate the range to which $\tan \phi \lesssim 1$ restricts $\phi$. We have 


$\mathrm{D} \ll \mathrm{L} \ll \mathrm{R}$,
or $\quad \frac{\mathrm{D}}{\mathrm{L}} \ll 1 \ll \frac{\mathrm{R}}{\mathrm{L}}$
or $\quad \frac{\mathrm{D}}{\mathrm{I}} \ll \tan \varphi<\frac{\mathrm{R}}{\mathrm{L}}$.

If we take $R=6000 \mathrm{~km}, L=1000 \mathrm{~km}, D=5 \mathrm{~km}$, we have

$$
\frac{\mathrm{D}}{\mathrm{L}}=0.005 \ll \tan \phi<<\frac{\mathrm{R}}{\mathrm{x}}=6 \text {, }
$$

or, taking $\frac{1}{10}$ as the order of smallness,

$$
0.05<\tan \varphi<.6
$$

so that

$$
3^{\circ}<\phi<31^{\circ}
$$

If $\tan \phi<<1$ ( $1 . \mathrm{e}_{0}$, very close to the equator) then we can no longer ignore the term $2 \Omega \omega_{c o l} \phi$ relative to $2 \Omega v \sin \phi$ in the u-equation although in some cases $2 \Omega$ woo $\phi$ can be ignored relative to $w \frac{\partial u}{\partial z}$. If tan $\phi \gg 1$ (near the poles) then we cannot ignore centripetal terms due to the curvature of the coordinate lines in the $u, v$ equations, and the term of simliar, origin In the equation of continutey.

The restriction of $\phi$ to a falrly narrow range auggests that, in the term $2 \Omega \sin \phi=f$, we can 1 inearize $\sin \phi$ about some $\phi=\varphi_{0}$ in the allowed interval: $\sin \left(\varphi-\varphi_{0}\right) \div\left(\varphi-\varphi_{0}\right), \cos \left(\varphi-\varphi_{0}\right) \div 1$. Then we have 


$$
\begin{aligned}
f & =2 \Omega \sin \phi=2 \Omega \sin \left[\left(\phi-\phi_{0}\right)+\phi_{0}\right] \\
& =2 \Omega \sin \left(\phi_{-}\right) \cos \phi_{0}+2 \Omega \sin \phi_{0} \cos \left(\phi_{0}-\phi_{0}\right) \\
& =2 \Omega\left(\phi-\phi_{0}\right) \cos \phi_{0}+2 \Omega \sin \phi_{0} \\
& =\frac{2 \Omega}{R}\left[R\left(\varphi_{-} \varphi_{0}\right)\right] \cos \phi_{0}+\phi_{0} \\
& =f_{0}+\beta y .
\end{aligned}
$$

where we have put $\beta=\frac{2 \Omega}{R} \cos \phi_{0}$, and $R\left(\phi-\phi_{0}\right)=y$; y measures north-south distance from the latitude $\phi_{0}$. In this way we can allow for the variation of the Coriolis parameter, f, with latitude, which is exceedingly important, without becoming too entangled in analytical difficulties.

$$
\text { Another consequence of the restriction of } \phi \text { to a fairly }
$$
narrow range of latitude is that we can take $\cos \phi \doteq \cos \phi_{0}$, nd so

$$
\begin{aligned}
& d x=R \cos \phi_{0} d \lambda, \\
& d y=R d \phi, \\
& d z=d r .
\end{aligned}
$$

that is, the differential equations relating one set of coordinates to the other become integrable, so that we can write

$$
\begin{gathered}
\frac{1}{r \cos \phi} \frac{\partial}{\partial \lambda}=\frac{\partial}{\partial x}, \\
\frac{1}{r \cdot \frac{\partial}{\partial \phi}}=\frac{\partial}{\partial y}, \\
\frac{\partial}{\partial r}=\frac{\partial}{\partial z} .
\end{gathered}
$$

Gathering up the previous results we find that the eq ations of motion are:

$$
\left(\frac{D}{D t}\right)_{r}^{u}-f v=-\frac{1}{p} \frac{\partial p}{\partial x}+\text { frictional forces, }
$$




$$
\begin{aligned}
&\left(\frac{D}{D t}\right)_{r}^{r}+f u=-\frac{1}{p} \frac{\partial p}{\partial y}+\text { frictional fonces } \\
& g=-\frac{1}{p} \frac{\partial p}{\partial z} . \\
& \frac{\partial u}{\partial x}+\frac{\partial v}{\partial y}+\frac{\partial u}{\partial z}=0 .
\end{aligned}
$$

These are the equations of motion relative to a rotating plane, but with $f=f_{0}+\beta_{y}$. This then is the $\beta$-plane approxination.

\section{A Word on Friction}

In the last few years direct messurements of ocean currents have been made by the neutrally buoyant float. This has indicated the likelihood of very large turbulent velocities, much greater than the average velocitles, in the ocean. This makes the assignment of eddy viscosities, so common in the past, seem foolish. However, there does not seem to be any reasonable alternative at present, so we shall continue to use the concept of eddy viscosity.

\section{Ekman's Theory of Wind-Drift.}

\section{Steady Flow}

We now turn to the theory of wind-driven ocean-currents, and follow the historical development. Ekman's theory deals with the manner in which the direct wind-stress effect penetrates into the ocean. We consider an ocean which is laterally unbounded, and uniform in all its properties. Thus no horizontal pressure or 
velocity gradients exist, since by assumption the only possible variation of veloclty is in the vertical; for the same reaon the non-linear terms drop out 21 so. We further asume a steady state. We assume the frictlonal force to be given by tems of the usual form, $\frac{1}{\rho} \operatorname{div}(\mu \operatorname{grad} \vec{V})$.

In fact, we will later want to replace $\mu$ by an eddy viscosity, $A$, and to do this me mut assume that the foregoing restrictions apply to the mean pressure and velocity flelds in a statistical steady flow. We ther obtain the equations of motion, expressing a balance between frtctional and Cortolis forces:

$$
\begin{aligned}
& -f v=\frac{1}{p} \frac{\partial}{\partial z}\left(\mu \frac{\partial u}{\partial z}\right), \\
& f u=\frac{1}{p} \frac{\partial}{\partial z}\left(\mu \frac{\partial v}{\partial z}\right) .
\end{aligned}
$$

The partial derivatives can be replaced by ordinary derivatives, and putting $w=u+i$ we have

$$
\frac{d^{2} w}{d z^{2}}-\frac{i f}{\nu} w, v=\frac{\mu}{p} .
$$

The solution of this equation is

$$
u=C e^{m z}
$$

with $m=\frac{\text { if }}{\gamma}$. The coordinate, $z$, is taken to be positive upward; hence to satisfy the condition that $W \rightarrow 0$ as $z \rightarrow-\infty$, we take $m=+\sqrt{\frac{i f}{\gamma}}$.

Thus, using $\sqrt{i}=(1+i) / \sqrt{2}$ we have 
$-81-$

$$
\begin{aligned}
w & =C e^{\sqrt{\frac{f}{2 \nu}}(1+i) z} \\
& =C e^{\sqrt{\frac{f}{2 \nu}}}\left[\cos \sqrt{\frac{F}{2 \nu}} z+i \sin \sqrt{\frac{f}{2 \nu}} z\right]
\end{aligned}
$$

To evaluate $C$ we use the following boundary conditions:

$$
\left(\mu \frac{\partial u}{\partial z}\right)_{z=0} \tau_{2},\left(\mu \frac{\partial v}{\partial z}\right)_{z=0}=0
$$

These conditions simply specify that at the surface the wind stress, $\tau$, is acting in the $x$-direction, and must be balanced by a corresponding u-velocity shear in the vertical in the water at the surface. Then in terms of w

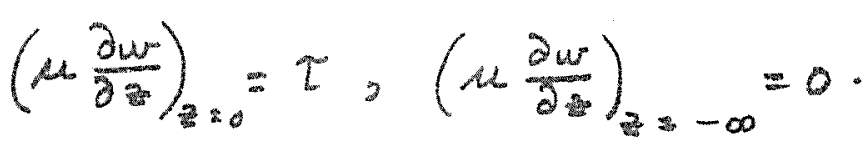

Now

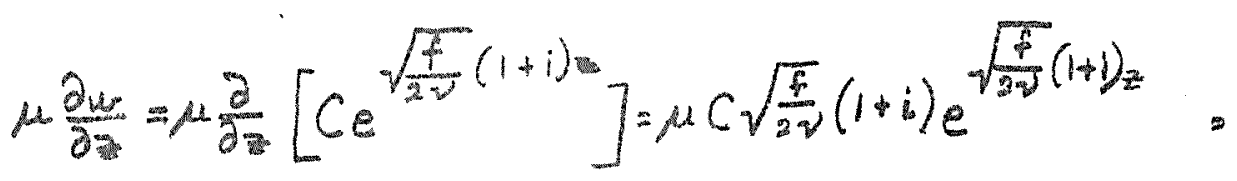

and 80

$$
\left(\mu \frac{\partial w}{\partial z}\right)_{z=0}=\mu C \sqrt{\frac{f}{\nu}} \frac{(1+i)}{\sqrt{2}}=\tau \text {. }
$$

Put $C=a e^{i b}$, and note that $\frac{1+i}{\sqrt{2}}=\left(\cos 45^{\circ}+i \sin 45^{\circ}\right)=e^{i \pi / 4}$, so that

$$
\frac{\mu}{p} a e^{i b} \sqrt{\frac{\rho}{\nu}} e^{i r / 4}=\frac{\tau}{\rho},
$$

or

$$
\frac{\nu a p}{\tau} \sqrt{\frac{f}{\nu}} e^{i\left(b+\frac{\pi}{4}\right)}=1 \text {. }
$$

Hence

$$
b+\frac{\pi}{4}=0 \text {, or } b=-\frac{\pi}{4} \text {, and } a=\frac{\tau}{p} \frac{1}{\sqrt{2}} \text {. }
$$


We thus find

$$
u r=\frac{\tau}{\rho} \frac{1}{\sqrt{\nu+}} e^{\sqrt{\frac{f}{2 \nu}}=} e^{i\left(2 \sqrt{\frac{f}{2 \nu}}-\frac{\pi}{4}\right)}=u+i v
$$

so

$$
\begin{aligned}
& u=\frac{\tau}{\rho} \frac{1}{\sqrt{v f}} e^{\sqrt{\frac{f}{2 \nu}} \cos \left(\frac{\pi}{2 v}-\frac{\pi}{4}\right)} . \\
& v=\frac{\tau}{p} \frac{1}{\sqrt{y f}} e^{\sqrt{\frac{f}{2 \nu}} \sin \left(z \sqrt{\frac{f}{2 \nu}}-\frac{\pi}{4}\right) .}
\end{aligned}
$$

Thts solution can be plotted in the $(u-v)$ plane for vartous $z$ to yield the Ekman spiral. This shows how the magnitude and direction of the wind-induced current change with depth.

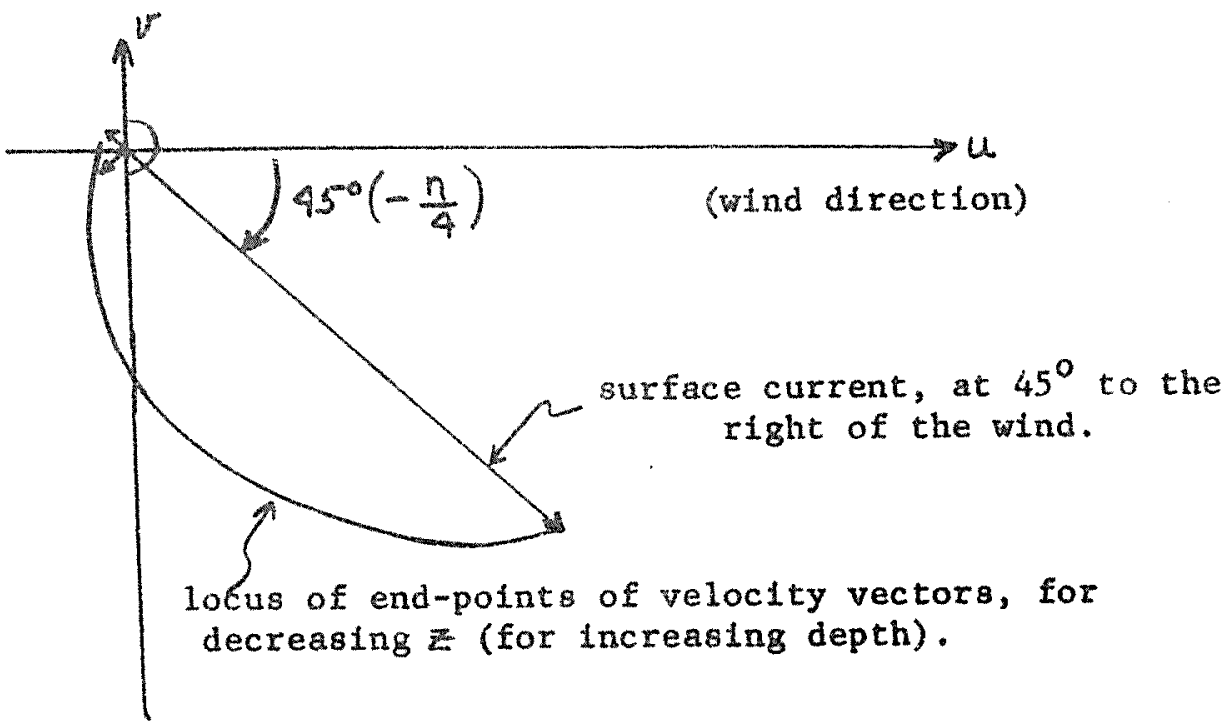

We note that at $\sqrt{\frac{f}{2 v}}-\frac{\pi}{4}=-\frac{3 \pi}{4}$, the flow is opposite to that at the surface. The depth at which this occurs is given by $-z=D$, with $-D \sqrt{\frac{f}{2 \nu}}=-\frac{3 n}{4}+\frac{\pi}{4}=-\frac{n}{2}$, so that $D=n \sqrt{\frac{2 \vartheta}{f}}$. At this depth the current is reduced by a factor 
$e^{-n}$ below its surface value. 0 is often called the "thickness of the Ekman layer". At mid-latitudes $\mathrm{E} \doteq 10^{-4} \mathrm{sec}^{-1}$, and $\mathrm{so}$, if we use for $\nu$ its molecular value, $0.01 \mathrm{cgs}$, we find $D=\frac{1}{2}$ metre. To obtain an appreclable depth for the Ekman layer, we must take eddy viscostty values for $\nu, i . e$. , replace $\nu$ by $A / P$. For $A / P=100 \nu$, and $10^{4} \nu$, we find $D=5$ metres, and 50 metres, respectively.

Note that if the Cortolis paraneter is neglected $(f=0)$, we find that the effect of viscosity penetrates unhindered to $z=-\infty$. The effect of Corlolis forces 18 then to limit the depth of penetration of viscous effects $(\sim D)$ to finite values. If we consider the integrated current, we have, from the equations of motion

$$
\begin{aligned}
\int_{z=-\infty}^{0} \rho u d z & =U=\text { total transport in direction of wind } \\
& =\frac{1}{f} \int_{-\infty}^{0} \frac{\partial}{\partial z}\left(A \frac{\partial v}{\partial z}\right) d z \\
& =\frac{1}{\rho f}\left[A \frac{\partial V}{\partial z}\right]_{z=-\infty}^{0} \\
& =0 \quad \text {, by the boundary conditions, }
\end{aligned}
$$

and

$\begin{aligned}-\int_{-\infty}^{0} p v d z=\nabla & =\text { total transport at } 90^{\circ} \text { to right of wind } \\ & =\frac{1}{f}\left[A \frac{\partial u}{\partial z}\right]_{-\infty}^{0}=\frac{\tau}{f}, \text { by the boundary conditions. }\end{aligned}$

Particularly note that this result is independent of the precise

functional form of $A$. This result can, of course, also be obtained 
by integrating the solutions we have obtajned for $u$ and $v$. This transport at $90^{\circ}$ to the right of the wind direction causes convergence and divergence in the Ekman layer, due to the varlability of the wind in space and time. Th1s convergence and divergence has profound influence on the motions of the deeper layers.

\section{Digression on Inertial rlow}

In the case where friction is absent, but the time dependence is retained, the equations of motion become:

$$
\begin{aligned}
\frac{\partial u}{\partial t}-f v & =0, \\
\frac{\partial v}{\partial t}+f u & =0 . \\
\text { or } \quad \frac{\partial u}{\partial t}+i f u & =0 .
\end{aligned}
$$

The solution of this equation is $\omega r=w_{0 e^{-i f t}}, w_{0}$ an arbitrary (real) constant, or

$$
\begin{aligned}
& u_{0}=\omega_{0} \cos f t, \\
& v=-w_{0} \sin f t .
\end{aligned}
$$

This motion is called "inertial" motion: the end-point of the velocity vector describes circles in the $(u-v)$ plane with period

$$
\begin{aligned}
& \frac{2 \pi}{f}=\frac{2 \pi}{2 \Omega \sin \phi} \text {. Now } \\
& \Omega=\frac{2 \pi}{\text { period of revolution of earth }}=\frac{2 \pi}{1 \text { sidereal day }} \text {. Thus, } \\
& \frac{2 \pi}{f}=\text { "inertial period" }=\frac{1}{2} \frac{2 \pi}{\Omega} \frac{1}{\sin \phi}=\frac{1}{2} \frac{\text { sidereal day }}{\sin \phi} .
\end{aligned}
$$


The quantity, $\frac{\text { sidereal day }}{\sin \phi}$ is called the "pendulum day", since it is the period of a Foucault pendulum at the latitude in question. Thus the "inertial period" equals $\frac{1}{2}$ pendulum days.

\section{The Time-Dependent Ekman Wind-Drift.}

The equations are the same as for the ateady-state case, with time-dependence included:

$$
\begin{aligned}
& \frac{\partial u}{\partial t}-f v=\frac{1}{\rho} \frac{\partial}{\partial z}\left(\mu \frac{\partial u}{\partial x}\right), \\
& \frac{\partial v}{\partial t}+f u=-\frac{\partial}{\rho} \frac{\partial}{\partial z}\left(\mu \frac{\partial v}{\partial z}\right) .
\end{aligned}
$$

The boundary conditions are as before, while the initial conditions are that at time $t=0$, when the wind stress is suddenly turned on, the currents in the water are zero. Again putting $w=u+i v$, we find

$$
\nu \frac{\partial^{2} w}{\partial z^{2}}-i f w-\frac{\partial w}{\partial t}=0, \nu=\frac{\mu}{p} \text {. }
$$

Taking the Laplace transform with respect to time,

$$
\begin{gathered}
\mathcal{L}\{w\}=\int_{0}^{\infty} e^{-5 t} u(t) d t \text {, we have } \\
\nu \frac{d^{2}}{d z^{2}} \mathcal{L}\{w\}-i f \mathcal{L}\{u r\}-s \mathcal{L}\{w\}+u(t=0)=0 \\
\text { or } \quad \frac{d^{2}}{d z^{2}} \mathcal{L}\{u r\}-\frac{(s+1 f)}{\nu} \mathcal{L}\{w\}=0 .
\end{gathered}
$$

The solution of this is

$$
\mathcal{L}\{w\}=C e^{\sqrt{\frac{s+i x}{v} z}}
$$

The boundary condition at $z=0 \mathrm{c}$ an be written:

$$
\mathcal{L}\left\{\left(\mu \frac{\partial w}{\partial z}\right)_{z=0}\right\}=\left(\mu \frac{\partial \mathcal{L}}{\partial z}\{w\}\right)_{z=0}=\mathcal{L}\{\tau\}=\frac{\tau}{s},
$$


$-86-$

and since $\quad\left(\frac{\partial \mathcal{L}}{\partial z}\{u r\}\right)_{z=0}=C \sqrt{\frac{s+i f}{v}}$.

We find $\frac{\mu}{p} C \sqrt{\frac{s+1 \gamma}{\nu}}=\frac{\tau}{s} \frac{1}{p}$

or

$$
C=\frac{\tau}{\rho \sqrt{v}} \frac{1}{s \sqrt{s+1}}
$$

Thus

$$
\mathcal{L}\{w\}=\frac{\tau}{\rho \sqrt{v}} \frac{1}{s \sqrt{s+i f}} e^{\frac{s}{\sqrt{2}}}
$$

We must now invert this to get the time dependence. The $\frac{1}{5}$ factor in $\mathcal{L}\{w\}$ simply indicates a time integration in the desired time-dependent function, so we look for the inverse transform of $\frac{1}{\sqrt{5+i f}} e^{-k \sqrt{5+i k}}$. This is found from the relation

$$
\mathcal{L}\left\{\frac{1}{\sqrt{\pi t}} e^{-k / 4 t}\right\}=\frac{1}{\sqrt{5}} e^{-k \sqrt{s}}, k \geqslant 0 \text {, and the rule that, }
$$

if $\mathcal{L}\{F(t)\}=\left\{(s)\right.$, then $\mathcal{L}\left\{e^{b t} F(t)\right\}=f(s-b)$.

Thus, with $b=$ if, we find

$$
\mathcal{L}\left\{\frac{1}{\sqrt{n t}} e^{-i f t} e^{-k / 4 t}\right\}=\frac{1}{\sqrt{s+i k}} e^{-k \sqrt{5+i /}}
$$

Then, as noted above, we use the rule that, if $\mathcal{L}\{F(t)\}=f(s)$, then $\mathcal{L}\left\{\int_{0}^{t} F(\alpha) \alpha \alpha\right\}=\frac{\{(s)}{s}$. We thus find, with $K=\frac{-5}{\sqrt{v}}$ ?

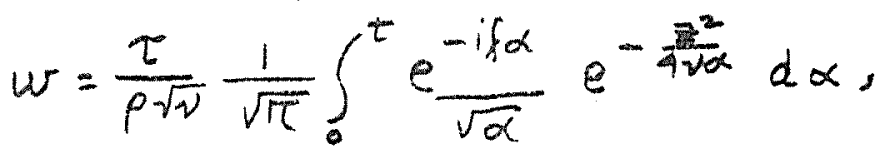

or,

$$
\begin{aligned}
& u=\frac{\tau}{\rho \sqrt{\nu}} \frac{1}{\sqrt{\pi}} \int_{0}^{t} \frac{\cos f \alpha}{\sqrt{\alpha}} e^{-\frac{t^{2}}{4 \sqrt{2} \alpha}} \alpha \alpha, \\
& u=-\frac{\tau}{\rho \sqrt{\nu}} \frac{1}{\sqrt{\pi}} \int_{0}^{t} \frac{\sin f \alpha}{\sqrt{\alpha}} e^{-\frac{z^{2}}{4 \sqrt{\alpha}} \alpha \alpha .}
\end{aligned}
$$


This solution can be plotted in the (u-v) plane for various a to show how the velocity at a given depth achieves its steady value.

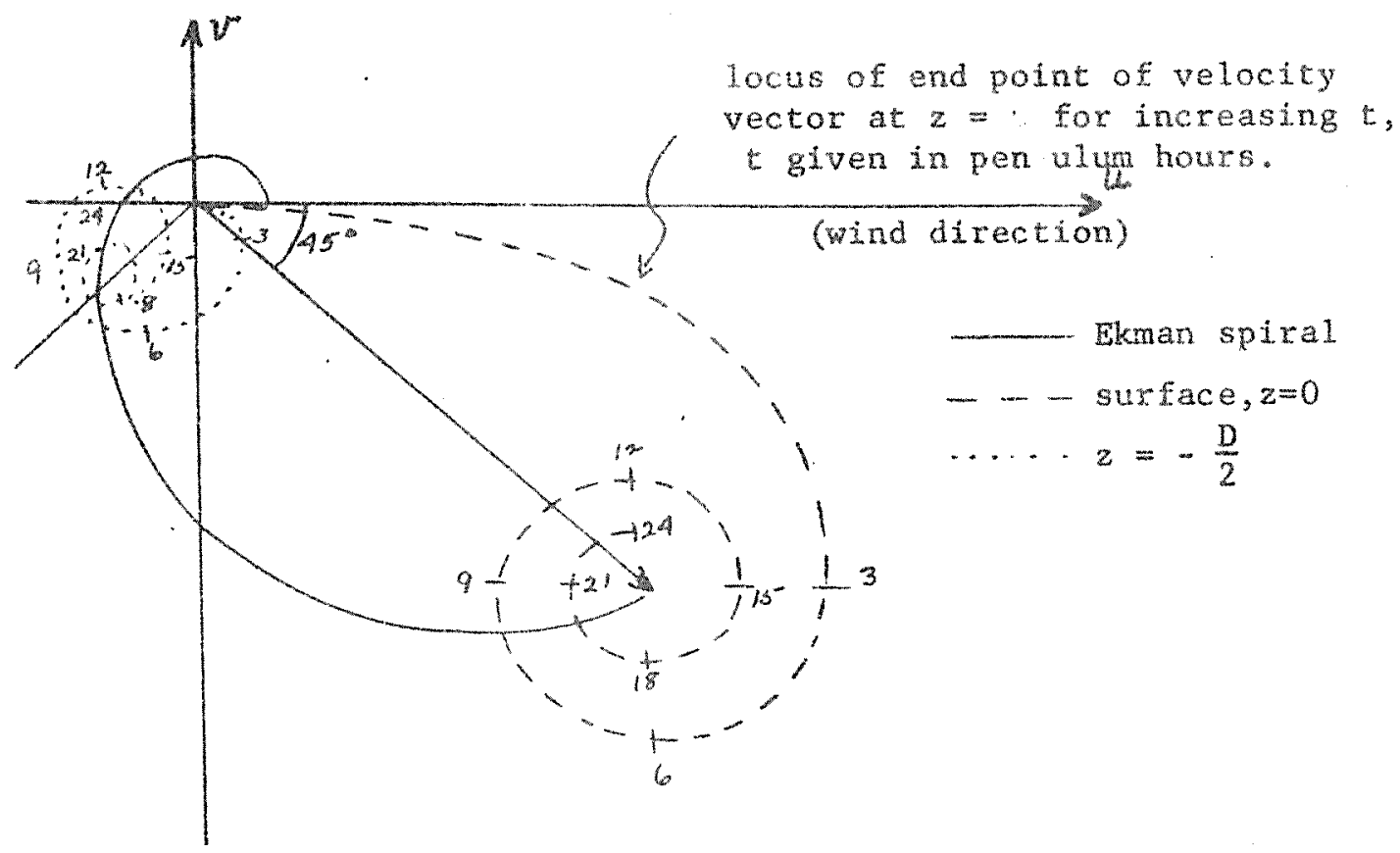

We see that in about a pendulum day the final ve ocity at any level is substantially reached.

We must note that the integrated current. in the transient solution does not converge, as $t \rightarrow \infty$, to that for the steady state as previously obtained. A general method of obtaining this result is as follows. Integrate the time-dependent equations of motion from $z=-\infty$ to 0 , to yield, according to previous definitions, 


$$
\begin{gathered}
\frac{\partial u}{\partial t}-f V=[\tau], \quad \begin{aligned}
{[\tau] } & =0 \text { for } t<0 \\
& =\tau \text { for } t>0
\end{aligned} \\
\frac{\partial v}{\partial t}+f u=0
\end{gathered}
$$

If we now write $U=U_{s}+U_{t} \quad V=V_{s}+V_{t}$ the subscripts $s$ and $t$ standing, respectively, for the steady and transient parts of the solution, we have

$$
\begin{aligned}
& \frac{\partial U_{t}}{\partial t}-f V_{s}-f V_{t}=[\tau], \\
& \frac{\partial V_{t}}{\partial t}+f U_{s}+f U_{t}=0 .
\end{aligned}
$$

Thus

$$
f U_{s}=0
$$

$$
f V_{s}=-\tau \text {, or } V_{s}=-\frac{\tau}{f} \text {, as before, }
$$

and

$$
\begin{aligned}
& \frac{\partial U_{t}}{\partial t}-f V_{t}=0, \\
& \frac{\partial V_{t}}{\partial t}+f U_{t}=0 .
\end{aligned}
$$

These are simply the equations for inertial motion; since these are non-decaying, there will always be an oscillating inertial component superposed on the steady transport, even at $t \rightarrow \infty$. This unrealistic behaviour can be eliminated by including a bottom friction boundary condition, or in fact any stress which w11 act in opposition to the total transport: the wind stress is at $90^{\circ}$ to the total transport, and so can do no work in damping the "transient" inertial motion of the total transport. In spite of this inertial, circular, motion of the whole water 
column, the inertial motion at any given depth can decrease steadily in time, because, as the motion penetrates to greater and greater depths, at any given level the contribution to the total inertial motion will be smaller and smaller.

\section{References:}

Ekman, V.W. Arkiv.f.Matem, Astr.o.Fysik (Stockholm) Bd.2, No.11, $53 \mathrm{pp} .(1905)$.

IV. Geostrophic Motion.

This mode of motion is governed by the following equation, representing a balance between Coriolis and pressure gradient forces:

$$
\begin{aligned}
& -f v=-\frac{1}{p} \frac{\partial p}{\partial x}, \\
& f u=-\frac{1}{p} \frac{\partial p}{\partial y} .
\end{aligned}
$$

The non-linear and frictional terms are omitted. It is observed that the main oceanic circulation is to a good approximation geostrophic, and the above equations form the basis for calculations of currents on the basis of measurements of salinities and temperatures. This method cannot yield absolute velocities unless a reference velocity at some depth is established. 
V. Sverdrup's Theory of Wind-Generated Transport.

Geostrophic motion, a presented above, shows no driving forces. We want to include the ind stress, and this wil be done by integrating the equation of motion up fxom some depth, so that the frictlonal forces evalunted at the gurface yleld the wind stress, an in the case of the calculation of the Ekman trangport. The Innovations here are the 1.ciulon of the pressure gradient, and the allowance for the varlactos of $f$ with latitude. This latter is a most fundamental point. More specifically we assume a statistically steady state, gnall velocities, lateral fiction negligible, surface elevations aegligible, and that the currents axe limited to some Einte depth. The equations of motion are:

$$
\begin{aligned}
-\rho f v & =-\frac{\partial p}{\partial x}+\frac{\partial}{\partial z}\left(A \frac{\partial u}{\partial z}\right) \\
\rho f u & =-\frac{\partial p}{\partial y}+\frac{\partial}{\partial z}\left(A \frac{\partial v}{\partial z}\right) .
\end{aligned}
$$

If we integrate from a depth $z=-D$ to the surface $z=0$, we have

$$
\begin{aligned}
-f V & =-\frac{\partial}{\partial x} P+\left[A \frac{\partial u}{\partial z}\right]_{-D}^{0}, \\
f U & =-\frac{\partial}{\partial y} P+\left[A \frac{\partial v}{\partial z}\right]_{-D}^{0}
\end{aligned}
$$

We have put $P=\int_{-\sigma}^{0} p d z$, and, as before, $\int_{-D}^{0} p u d z=U, \int_{-D}^{0} p v d z=V$, except that now we have a lower linit at $z=-D$ rather than 
$z=-\infty$. We assume that the stress at $z=-D 1 s$ zero, in accordance with the assumption of zero current below sone depth, so that $\left[A \frac{\partial u}{\partial z}\right]_{-D}^{0}=\tau_{x}-0=\tau_{x},\left[A \frac{\partial v}{\partial z}\right]_{-D}^{0}=\tau_{y}-0=\tau_{y}$ with $\tau_{x}, \tau_{y}$ being the wind stress at the surface. Thus

$$
\begin{aligned}
& -f V=-\frac{\partial P}{\partial x}+\tau_{x} \\
& f u=-\frac{\partial P}{\partial y}+\tau_{y}
\end{aligned}
$$

The equation of continuty, $\operatorname{div}(\rho \vec{V})=0$, on integration from $z=-D$ to $z=0$, yields

$$
\frac{\partial u}{\partial x}+\frac{\partial v}{\partial y}=0
$$

We now take the curl of the equations of motion, or, equivalently, form

$$
\begin{gathered}
-\frac{\partial}{\partial y}(-f V)+\frac{\partial}{\partial x}(f u)=\frac{\partial^{2} p}{\partial y \partial x}-\frac{\partial^{2} p}{\partial x \partial y}-\frac{\partial \tau_{x}}{\partial y}+\frac{\partial \tau_{y}}{\partial x} \\
\text { or } V \frac{\partial f}{\partial y}+f \frac{\partial V}{\partial y}+U \frac{\partial f}{\partial x}+f \frac{\partial u}{\partial x}=\frac{\partial \tau_{y}}{\partial x}-\frac{\partial \tau_{x}}{\partial y} \\
\text { or } \beta V=(\operatorname{curl} \vec{\tau})_{z}=z \cdot \text { component of cur1 } \vec{\tau},
\end{gathered}
$$

since $\frac{\partial f}{\partial y}=\beta, \frac{\partial f}{\partial x}=0$, and $\frac{\partial \gamma}{\partial y}+\frac{\partial u}{\partial x}=0$.

Thus $V=\frac{1}{\beta}\left(\operatorname{cur}(\vec{\tau})_{z}\right.$, or, the north-south mass transport is completely determined by the curl of the wind stress.

If we substitute this relation into the continuity equation, and integrate with respect to $x$, we find: 


$$
\lambda=-\frac{1}{\beta} \int \frac{\partial}{\partial y}(\operatorname{sul} \vec{z})_{z} d x+g(y)
$$

g(y) is ar arbitrary sunction of $y$, which we non must evaluate.

Since our equations are of first order, essentially, we car satisfy orly che boundary condition, and by the natuxe of our approximattors, which are not likely to hold in the western parts of oceans where rapd, interse currents occur, we restrict curselves to the region of the eastern boundary, say from $x=E$ to $x=L$, whers $E$ is some distance away from the western rcusdary, $\quad x=0$.

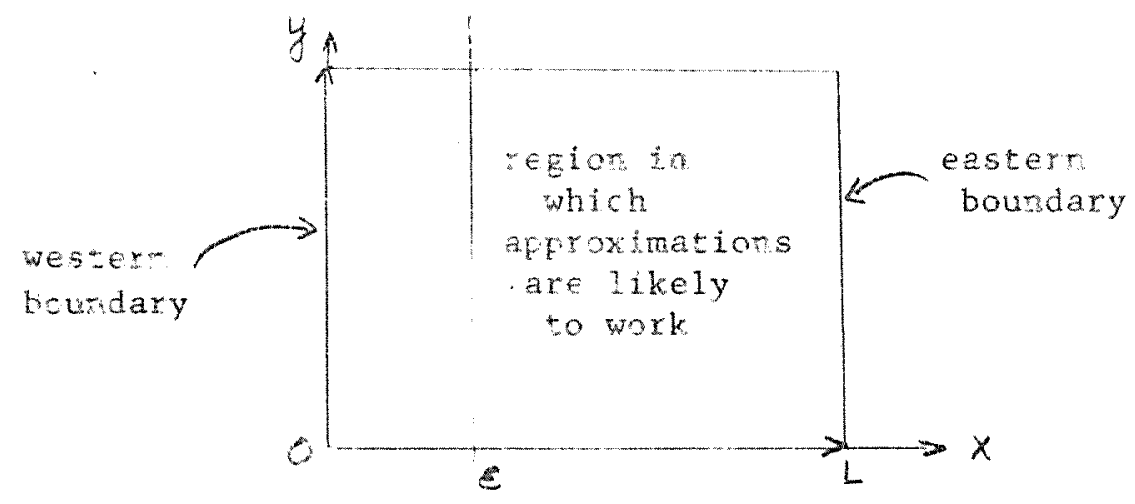

Ther, at $x=L$, west have $U=0$, for ali y, Thus, since

$$
\begin{aligned}
& \frac{\partial U}{\partial x}=-\frac{\partial}{\partial y} \frac{1}{\beta}(\operatorname{cish} \vec{\tau})_{z} \text {, we have } \\
& \int_{\epsilon}^{L} \frac{\partial u}{\partial x} d x=-\frac{1}{\beta} \int_{\varepsilon}^{L} \frac{\partial}{\partial y}(\cos \theta \vec{z})_{z} d x \\
& =U(L)-U(\epsilon) \\
& =0-u(\epsilon) \text {. }
\end{aligned}
$$

Now $E$ is any alue of $x$ urom some distance out from $x=0$ :0 


$$
\begin{aligned}
& x=L \text {, so we can write } \mathcal{L}(\epsilon)=U(x), L>x>E \text {, and } \\
& U(x)=\frac{1}{\theta} \int_{x}^{\log } \frac{\partial}{\partial y}(\operatorname{cul} / \vec{T}) d x \\
& -\frac{1}{\beta} \int_{L}^{x} \frac{\partial}{\partial y}\left(\operatorname{cus} t^{t}\right) d x
\end{aligned}
$$

If we have only zonal winds, so that $\left.\tau_{y}=0,(\omega \mathrm{m}) z_{z}\right)=-\frac{\partial \tau_{x}}{\partial y}$, and if $t_{x}$ is independent of $x$, then

$$
L(x)=\frac{\partial^{2} \tau_{x}}{\partial y^{2}} \frac{x-L}{\beta} .
$$

Sverdrup applied thls relation to the equatorial counter-current region of the south pacific, and obtained reasonable agreement as far as the relative directions and relative magnitudes of the currents were concerned. The absolute magnitudes estimated were not in good agreement with observation.

\section{Reference:}

Sverdrup, H.U. Proc. Nat. Acad. Scl., Wash., $33(1947), 318$. 


\section{Stomel's Theory of Western Intensification}

We now seek possible circulations in closed basins, which was not possible using Sverdrup's approximations. The crucial point is the introduction of a frictional term, in the form of a drag on the ocean bottom: whether or not this is a realistic source of dissipation is secondary to the demonstration that closed circulations with western intensification are . possible with the inclusion of such a term.

We consider a rectangular basin, $0 \leqslant x \leqslant L, 0 \leqslant y \leqslant b$, and $a$ wind stress given by $\tau(y)=-F \cos (\pi y / b)$. We further $\int_{-D}^{h} \frac{\partial}{\partial z}\left(A \frac{\partial u}{\partial z}\right) d z=\left(A \frac{\partial u}{\partial z}\right)_{-D}^{h}=\left(\tau_{x}\right)_{h}-\left(\tau_{x}\right)_{D}$, and similarly for $v,\left(\tau_{y}\right)_{h}=\left(\tau_{y}\right)_{D}$, where the following diagram illustrates the notation:

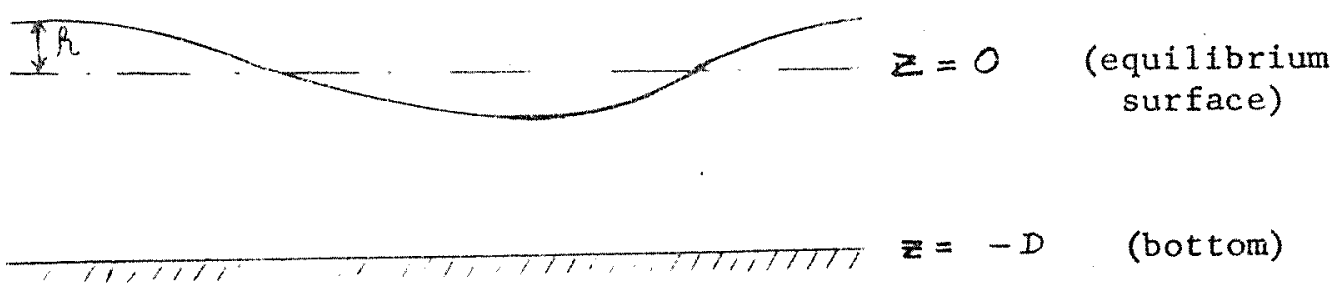

We now choose

$$
\begin{aligned}
& \frac{\left(\tau_{x}\right)_{R}}{\rho}=\frac{\tau(y)}{\rho}=-F \cos (\pi y / b), \\
& \frac{\left(\tau_{y}\right)_{h}}{\rho}=0, \\
& \frac{\left(\tau_{x}\right)_{D}}{\rho}=R \bar{u}, \\
& \frac{(\tau y)_{D}}{\rho}=R \bar{v},
\end{aligned}
$$


with $\bar{u}=\frac{1}{D} \int_{-D}^{h} u d z, \bar{v}=\frac{1}{D} \int_{-D}^{h} u d z$, and $R$ is a constant measuring the magnitude of the bottom drag. This assumption of a non-zero bottom drag is the point which allows deductons different from those of previous theories.

The equations of motion are:

$$
\begin{aligned}
& -f v=-\frac{1}{\rho} \frac{\partial p}{\partial y}+\frac{1}{\rho} \frac{\partial}{\partial z}\left(A \frac{\partial u}{\partial z}\right), \\
& f u=-\frac{1}{\rho} \frac{\partial p}{\partial y}+\frac{1}{\rho} \frac{\partial}{\partial z}\left(A \frac{\partial v}{\partial z}\right), \\
& -\frac{1}{\rho} \frac{\partial p}{\partial z}=g, \\
& \frac{\partial u}{\partial x}+\frac{\partial v}{\partial y}+\frac{\partial w}{\partial z}=0 .
\end{aligned}
$$

For homogeneous water, we have $p=p g(h-2)$.

We integrate now from $z=-D$ to $z=h$, putting by definition $\int_{-D}^{h} u d z=\bar{u}(D+h)=D \bar{u}$, and noting that

$$
\begin{aligned}
-\int_{-D}^{h} \frac{1}{\rho} \frac{\partial p}{\partial x} d z & =-\frac{1}{\rho} \int_{-D}^{h} \rho g \frac{\partial h}{\partial x} d z=-g \frac{\partial h}{\partial x} \int_{-D}^{h} d z \\
& =-g \frac{\partial h}{\partial x}(h+D) \pm-g D \frac{\partial h}{\partial x},
\end{aligned}
$$

with similar relations for $\int_{-0}^{h} v d z,-\int_{-0}^{h} \frac{1}{\rho} \frac{\partial p}{\partial x} d z$, so that the equations become

$$
\begin{gathered}
-f D \bar{v}=-g D \frac{\partial h}{\partial x}+(-F \cos (\pi y / b))-R \bar{u}, \\
f D \bar{u}=-g D \frac{\partial h}{\partial y}+(-R \bar{v}), \\
\frac{\partial \bar{u}}{\partial x}+\frac{\partial \bar{v}}{\partial y}=0 .
\end{gathered}
$$


Taking the curl of the equations of motion yields

$$
\frac{D \beta}{R} \vec{v}+\frac{\pi F}{R b} \sin \left(\frac{\pi y}{b}\right)+\frac{\partial \vec{v}}{\partial x}-\frac{\partial \bar{u}}{\partial y}=0 \text {. }
$$

We introduce a stream function $\psi$ so that $\psi_{x}=\bar{v}$, $\Psi_{y}=-\bar{u}$, and the continuity equation is identically satisfied. Then the last equation becomes

$$
\frac{D_{\beta}}{R} \frac{\partial \psi}{\partial x}+\frac{\pi F}{R b} \sin \left(\frac{\pi y}{b}\right)+\frac{\partial^{2} \psi}{\partial x^{2}}-\left(-\frac{\partial^{2} \psi}{\partial y^{20}}\right)=0,
$$

or, with

$$
\begin{gathered}
\alpha=\frac{D \rho}{R}, \gamma=\frac{\pi F}{R b}, \\
\nabla^{2} \psi+\alpha \psi_{x}=\gamma \sin \left(\frac{\pi y}{b}\right)
\end{gathered}
$$

If we assume a $y$-dependence for $\psi$ of the form $\sin \left(\frac{\pi y}{L}\right)$, that is, if we put $\psi(x, y)=f(x) \sin \left(\frac{\pi y}{b}\right)$, then the foregoing equation yields

$$
\begin{array}{ll}
\sin \left(\frac{\pi y}{b}\right) \frac{d^{2} f}{d x^{2}} & +f(x)\left(-\sin \left(\frac{\pi y}{b}\right)\right)\left(\frac{\pi}{b}\right)^{2}+\alpha \frac{d f}{d x} \sin \left(\frac{\pi y}{b}\right)=8 \sin \left(\frac{\pi y}{b}\right), \\
\text { or } \quad & f^{\prime \prime}+\alpha f^{\prime}-\left(\frac{\pi}{b}\right)^{2} f=\gamma .
\end{array}
$$

The general solution of the homogeneous equation, $f^{\prime \prime}+\alpha f^{\prime}-\left(\frac{\pi}{b}\right)^{2} f=0$, is gotten by substituting $e^{m x}$; then $m^{2}+\alpha m-\left(\frac{\pi}{b}\right)^{2}=0$, so $m=-\frac{\alpha}{2} \pm \sqrt{\frac{\alpha^{2}}{4}+\left(\frac{\pi}{b}\right)^{2}}$. Thus the solution of the homogeneous equation, that is, the complementary function, is

$$
P e^{A x}+Q e^{B x} \text {, }
$$


$-97-$

with $A=-\frac{\alpha}{2}+\sqrt{\frac{\alpha^{2}}{4}+\left(\frac{\pi}{b}\right)^{2}}, B=-\frac{\alpha}{2}-\sqrt{\frac{\alpha^{2}}{4}+\left(\frac{\pi}{b}\right)^{2}}$. $P, Q$ are constants to be used to satisfy the boundary conditions.

A particular solution of the nonhomogeneous equation, that is, the particular integral, can be got by trial. We try a constant, which on substitution into the equation gives

$$
-\left(\frac{\pi}{b}\right)^{2} \text { (particular integral }=\text { constant) }=\gamma
$$

or particular integral $=$ constant $=-\gamma\left(\frac{b}{\pi}\right)^{2}$.

The general solution of the nonhomogeneous equation is then

$$
f=-\beta\left(\frac{b}{n}\right)^{2}+P e^{A x}+Q e^{8 x},
$$

and hence

$$
\begin{aligned}
& \psi \psi=f\left(\frac{b}{\pi}\right)^{2}\left[p e^{A x}+q e^{B x}-1\right] \sin \left(\frac{\pi y}{b}\right), \\
& \text { with } \quad p=\frac{p}{\gamma\left(\frac{b}{\pi}\right)^{2}}=q=\frac{Q}{\delta\left(\frac{b}{\pi}\right)^{2}} .
\end{aligned}
$$

We now choose as boundary conditions that $\psi=0$ on the rectangular boundary given by $y=0, b, x=0, L$. The condition on $y=0, b$ is met in virtue of the appearance of $\sin \left(\frac{\pi y}{b}\right)$ in $\psi$. The condiction on $x=0, L$ yields:

$$
\begin{gathered}
0=\delta\left(\frac{b}{\pi}\right)^{2}[p+q-1] \sin \left(\frac{\pi y}{b}\right): \\
a=\delta\left(\frac{b}{\pi}\right)^{2}\left[p e^{A L}+q e^{B L}-1\right] \sin \left(\frac{\pi y}{b}\right), \\
p+q-1=0 \\
p e^{A L}+q e^{B L}-1=0
\end{gathered}
$$

or 
so that $\quad p=\frac{1-e^{B L}}{e^{A L}-e^{B L}}, \quad q=\frac{e^{A L}-1}{e^{A L}-e^{B L}}$

Then we have

$$
\begin{aligned}
& \bar{u}=-\psi_{y}=-\beta\left(\frac{b}{\pi}\right)\left[p e^{A x}+q e^{B x}-1\right] \cos \left(\frac{\pi y}{b}\right), \\
& \bar{v}=\psi_{x}=\delta\left(\frac{b}{\pi}\right)^{2}\left[p A e^{A x}+q B e^{B x}\right] \sin \left(\frac{\pi y}{b}\right) .
\end{aligned}
$$

To interpret this solution, Stommel chose $L=10^{4} \mathrm{~km}$, $b=2 \pi \times 10^{3} \mathrm{~km} ., \mathrm{D}=200$ metres, $\mathrm{R}=0.02, \mathrm{~F}=1 \mathrm{dyne} / \mathrm{cm}^{2}$. These values are arbitrary to arge extent, especially R, but the main point is to see the sort of results which emerge, The following graphs show these results.

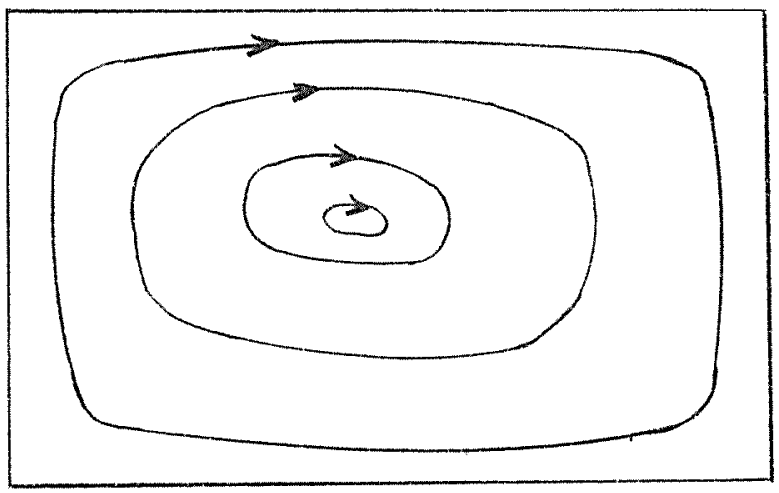

lines of $\psi$ constant, for $f=0$, $f=$ constant.

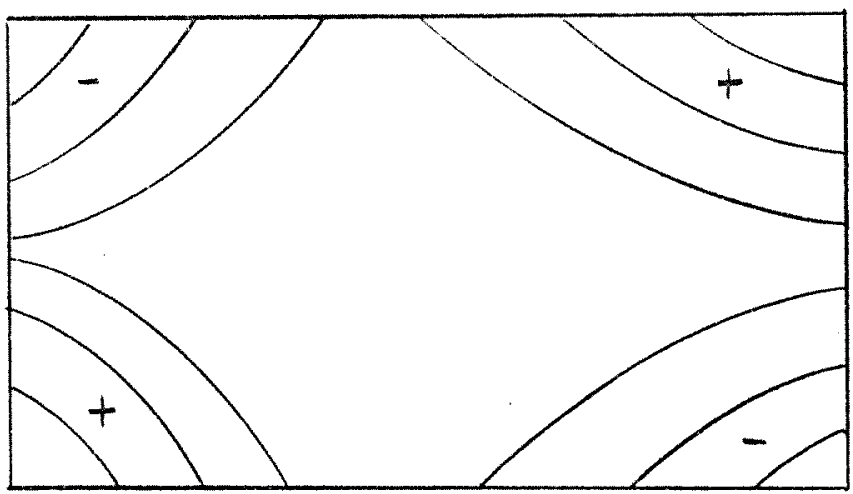

lines of constant elevation, for $f=0$ 


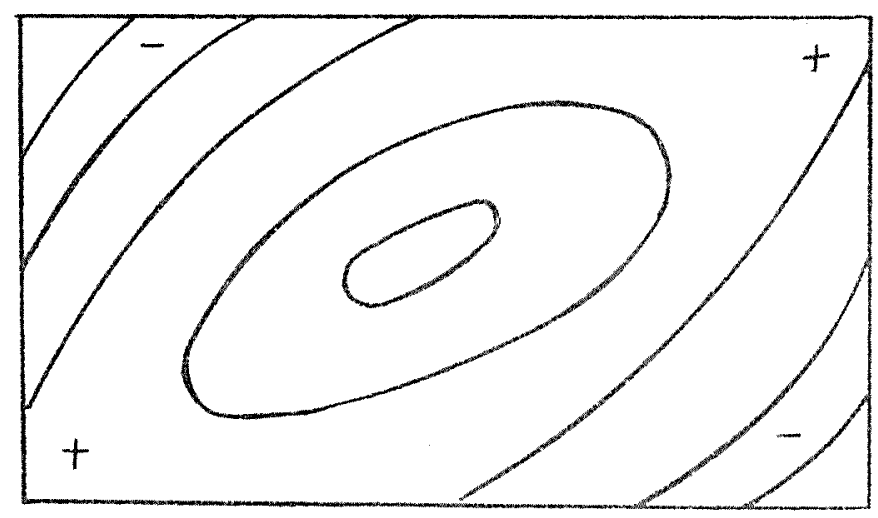

lines of constant elevation, for $f=$ constant $\neq 0$.

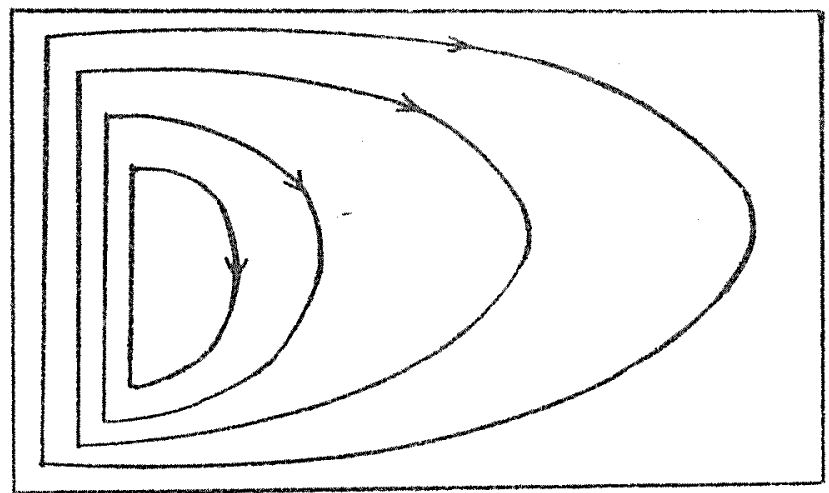

lines of $\psi$ s constant, for $f=$ constant $\neq 0$ $(\beta=$ constant $\neq 0)$.

The westward intensification is clearly shown for the cases $\beta \neq 0,1 . e .$, when the change of $f$ with latitude is taken into account.

References:

Stome1, H., 1948. Trans.Am.Geo.Union, 29, 202.

submitted by L.W.MacMillan and M.Mork. 
VII Munk's Theory of the Wind-driven Ocean Circulation.

1. The Assumptions and Formulations.

In this lecture we will consider another frictional theory of the ocean with a model structure similar to that of Stomel's. Munk's motivation to undertake this revision came from the fact that stommel's assumption of a $\bar{\mu}$ on the botton of the ocean was unrealistic. Munk treats the problem by using the following assumptions:

a). At some depth $z=-D$ all currents vanish and the stress is negligible.

b). The lateral frictional stress is not negligible.

c). The mean velocities are steady in the statistical sense.

d). The mean veloctty is everywhere small so that non Inear terms of the mean flow in the equation of motion can be neglected.

(Notice that assumptions $a$ and $b$ differ from Stomel's.) The equations of motion for this system are

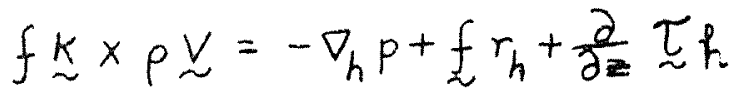

where $f r_{h}$ is the term due to lateral friction, and $h$ refers to the horizontal component of the operator.

The continuity equation is

$$
\nabla \cdot p \underline{v}=0
$$


Munk also uses the concept of vertically integrated mass transport which was introduced by sverdrup, and

$$
U=\int_{-D}^{\eta} \rho u d z, \quad V=\int_{-D}^{\eta} p v d z
$$

where $\eta$ is the surface elevation above $z=0$

The vertically integrated continuity equation is

$$
u, x+V, y=0
$$

The vertically integrated equations of motion are

$$
f k \times V-\nabla_{h} p+\int_{D}^{n} f r_{h}+\tau
$$

In the derivation of (78) we have used the following assumptions and definitions:

a) The stress vector $\tau$ is the horizontal stress vector $\tau_{h}$ evaluated at the surface $?$.

b) $\frac{\partial}{\partial x} \int_{-D}^{r} r d z=\int_{-D}^{\eta} \frac{\partial p}{\partial x} d z+\frac{\partial \eta}{\partial x} p \eta$

and the last term is negligible because the slope of the surface $\left(\frac{\partial \eta}{\partial x}\right)$ is small.

c)

$$
P=\int_{-D}^{z} p d z
$$

If the frictional term $\int_{-D}^{\eta} f^{-D} r_{h}$ is crudely approximated by $A \nabla_{h}^{2} \underset{V}{ }$, equation (78) can be written as:

$$
f \underset{\sim}{K} \times \underline{V}=-\nabla_{h} P+A \nabla_{h}^{2} \underset{\sim}{V}+\tau
$$

To simplify equation (79) we take its curl and use the 
continuity equation to obtain,

$$
V \beta=A \nabla^{2}(\nabla \times \underline{V})_{z}+(\nabla \times t)_{z}
$$

It is, furthermore, convenient to introduce the

stream function

$$
\psi_{, x}=V,-\psi, y=U
$$

and equation $(80)$ can be written as,

$$
A \nabla^{2} \nabla^{2} \psi-\beta \psi, x=-\operatorname{cur} \psi_{2}
$$

\section{Munk's Solution}

This equation is now applied to the rectangular ocean shown in Fig. 1. This Eigure shows only half of the

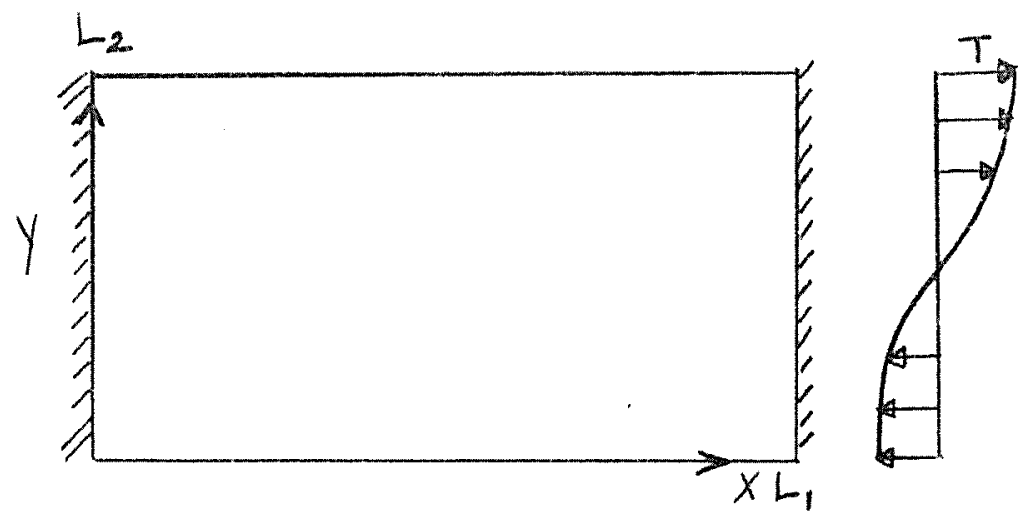

basin. The wind stress is applied over the whole cosine wave in the general case.

The boundary conditions on this model are
a) $\operatorname{curl} \tau=0$ at $y=0, L_{2}$
b) $\quad \psi=0$ on all the boundaries. 
c) $\psi_{1 X}=0 \quad$ at $X=0, \mathrm{~L}_{1}$

d) $\psi_{y_{y}} \equiv \frac{\partial U}{\partial y}=0$ at $y=0, L_{2}$, a condition satisfied by the forced interior solution.

Munk looked for boundary layer solutions of (81).

The motivation to do this comes from the physics of ocean circulation in which it is expected that friction plays a minor role in the interior of the ocean, i.e. A is very small. Now a proper non-dimensionalization, consistent with the reasoning above, is sought. Fig. I shows that the wind stress can be represented by

$$
\tau_{x}=-T \cos \frac{\pi y}{L_{2}}
$$

and

$$
- \text { curl, } z \tau=\frac{\pi T}{L_{2}} \sin \frac{\pi y}{L_{2}}
$$

This, of course, satisfies a). Then we can write, for the interior of the ocean basin the inertial balance equation

$$
\beta \psi_{1 x}=\operatorname{curl}_{\mathrm{z}} \tau=-\frac{\pi T}{L_{2}} \sin \pi \frac{\mathrm{V}}{L_{2}}
$$

This leads us to choose the non-dimensional variable

$$
\psi^{\prime} \text { as }
$$

$$
\psi=\frac{\pi T}{\beta} \psi^{\prime}
$$

Also introduce

$$
x=L_{2} x^{\prime}, y=L_{2} y^{\prime}
$$

and transform the boundaries by making $\frac{L_{1}}{L_{2}}=r$. Thus, the 
boundaries are $x=0, r ; y=0,1$.

We can write equation (81) as (dropping the primes)

$$
\in \nabla^{4} \psi-\psi_{1} x=\sin \pi y
$$

where

$$
\epsilon=\frac{A}{\beta L_{2}^{3}}
$$

Some observations indicate that, when the non-linear

terms are included in an "all compassing" frictional term as we have done, $10^{6} \leq A \leq 10^{8} \mathrm{~cm}^{2} \mathrm{sec}^{-1}$. With $\beta=2 \times 10^{-13} \mathrm{~cm}^{-1} \mathrm{sec}^{-1}$, $L_{2}=5 \times 10^{8} \mathrm{~cm}$, and $A=10^{8} \mathrm{~cm}^{2} \mathrm{sec}^{-1}$ we find $\in \sim 10^{-5}$. To get $A \sim 10^{8}$ we need eddies of the size of the meanderings of the Gulf Stream.

Hence, hopefully, Munk's theory can apply to the "climatological mean" boundary current.

Certain measurements in the Gulf Stream of A from the relation $\overline{\mathcal{V}^{\prime} \nabla \mathcal{V}^{\prime}}=A \frac{\partial^{2} \bar{v}}{\partial x^{2}}$ indicate that $A$ can even be negative. This would mean that the eddies are feeding energy to the mean stream. Munk's eddies draw energy from the stream. The short table below serves to illustrate the above points.

$$
\text { Consider the terms } \beta \frac{\partial \psi}{\partial x} \quad A \frac{\partial \psi}{\partial x^{2}} \text {, and } \frac{\partial \psi}{\partial y} \frac{\partial^{3} \psi}{\partial x^{3}}
$$

which are of the order $\epsilon^{-1 / 3} \quad \epsilon^{-1 / 3}$ and $\epsilon^{-1} \frac{\pi T}{D \beta^{2} h^{3}}$. With $\mathrm{A}=10^{8}$ these become $\sim 10^{2} \sim 10^{2}$ and 5 but with $A=10^{6}$ these become $\sim 3 \times 10^{2} 3 \times 10^{2}$ and $3 \times 10^{2}$.

Hence, neglecting the non-linear terms is consistent for large A but is incorrect for small A. 
Let us now assume that $\psi$ is of the form $\sin \pi y$ as a $y$ variation. We can then write the $x$-dependent part of equation (84) as

$$
\epsilon\left[\frac{d^{4} \psi}{d x^{4}}+2 \pi \frac{d^{2} \psi}{d x^{2}}+\pi^{4} \psi\right]-\frac{d \psi}{d x}=1
$$

Let $\psi_{i}$ be the solution in the interior of the basin, then from (85)

$$
\psi_{i}=-x+\text { const. }
$$

Near the boundary frictional terms are important and since this boundary "layer" is very small, we will "stretch" the $X$ coordinate in this region by introducing the variable $\xi$ defined as

$$
x=\epsilon^{n} \xi
$$

and $\eta$ is a constant. The value of $\eta$ can be determined from equation (85). Since we require the balance in the boundary (because of boundary conditions) to retain $\frac{d^{4} \psi}{d x^{4}}$ term, we say that this is of order unity in the boundary region. We now match this term with all others in the equation which are of order unity. If this is done in turn with all combinations of terms in (85) we find that the balance is between $\frac{d^{4} \psi}{d \xi^{4}}$ and $\frac{d \psi}{d \xi}$.

The transformed equation (85) is

$$
\epsilon^{1-4 n} \frac{d^{4} \psi}{d \xi^{4}}-2 \pi \epsilon^{1-2 n} \frac{d^{2} \psi}{d \xi^{2}}+\epsilon \pi^{4} \psi-\epsilon^{-n} \frac{d \psi}{d \xi}=1
$$

and the abowe balance yields

$$
\begin{aligned}
1-4 n & =-n \\
n & =1 / 3 .
\end{aligned}
$$


Then we can write

$$
\frac{d^{4} \psi}{d \zeta^{4}}-2 \pi^{2} \epsilon^{3 / 3} \frac{d^{2} \psi}{d \zeta^{2}}+\epsilon^{4 / 3} \pi^{4} \psi-\frac{d \psi}{d \zeta}=\epsilon^{1 / 3}
$$

and, to an error of order $\epsilon^{1 / 3}$, this equation can be approximated by

$$
\frac{d^{4} \psi}{d \zeta^{4}}=\frac{d \psi}{d \xi}
$$

for the boundary layer.

Let $\psi_{b}$ be the solution of $(88)$, then

$$
\psi_{b}=C_{1}+c_{2} e^{\zeta}+C_{3} e^{\zeta e^{\frac{2 \pi i}{3}}}+C_{4} e^{\zeta e^{\frac{4 \pi i}{3}}} .
$$

The actual solution is $\psi=\psi,+\psi$, , and, therefore, we require that $\psi_{b} \rightarrow 0$ as $\zeta \rightarrow \infty$ giving $c_{1}=c_{2}=0$.

Near $X=0$, the solution thus is

$$
\psi=-x+c+c_{3} e^{5 e^{\frac{2 \pi}{3}}}+C_{4} e^{5 \frac{4 T . j}{3}}
$$

At the other boundary $\chi=r$ we put $x-r=\varepsilon^{n} \zeta$ and arrive at a similar solution as equation (89) with the boundary condition that $\psi_{b} \rightarrow 0$ as . $\zeta \rightarrow-\infty$ which makes $c_{1}=c_{3}=c_{4}=0$. Near $\chi=r$, therefore,

$$
\psi=-x+c+c_{2} e^{(x-r) \epsilon^{-1 / 3}}
$$

The final solution of ( 88 ) then becomes 


$$
\begin{gathered}
\psi=\psi_{b(x=0)}+\psi_{b(x=r)}+\psi_{i} \\
\psi=-x+c+C_{3} e^{e^{-1 / 3} e^{\frac{2 \pi i}{3} x}}+C_{4} e^{e^{-1 / 3} e}+C_{2} e^{(x-r) \epsilon^{-1 / 3}}
\end{gathered}
$$

If we, furthermore, apply the boundary conditions

b) and c) the complete solution of (81.) is

$$
\begin{aligned}
\psi & =\sin \pi y\left\{-x+r-\epsilon^{1 / 3}+\epsilon^{1 / 3} e^{(x-r) \epsilon^{-1 / 3}}+\right. \\
& \left.+\left[\left(\epsilon^{1 / 3}-r\right) \cos \frac{x \sqrt{3} \epsilon^{-1 / 3}}{2}+\left(\sqrt{3} \epsilon^{1 / 3}-\frac{r}{\sqrt{3}}\right) \sin \frac{x \sqrt{3} \epsilon^{-1 / 3}}{2}\right] e^{-\frac{x \epsilon^{-1 / 3}}{2}}\right\}
\end{aligned}
$$

\section{The Representations of Results}

The form of the streamlines is shown on Fig. 2, and the transport velocities on the western boundary on Fig. 3.

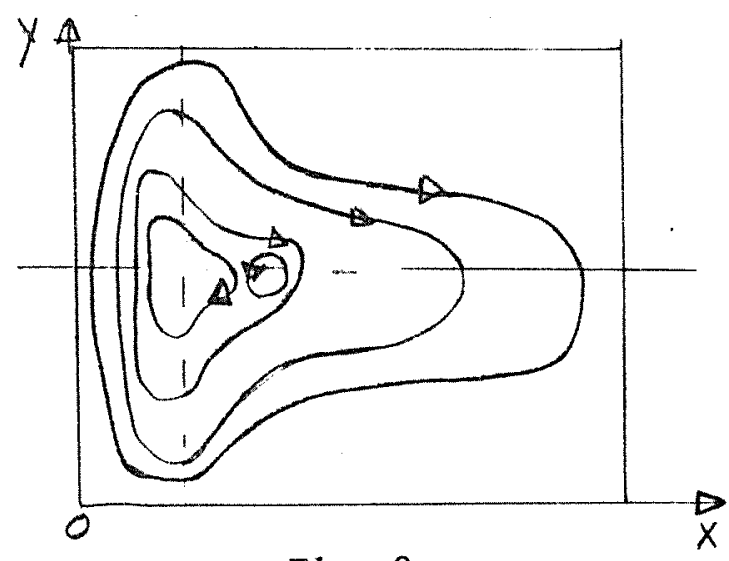

Fig. 2

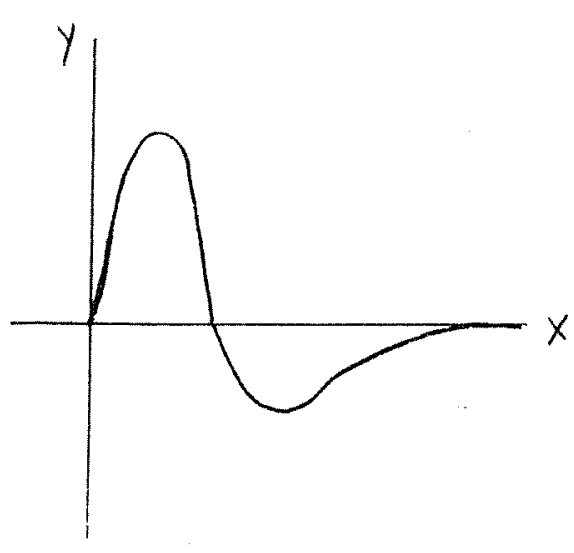

Fig. 3

Notice that Munk's theory predicts a countercurrent some distance from the boundary. The transport within this 
current is not negligible, but amounts to about $20 \%$ of the northward transport to the west.

The contour lines of the $\eta$ elevations, 1 .e. the surface, corresponding to the full cosine curve of $t_{x}$ are shown on Fig. 4.

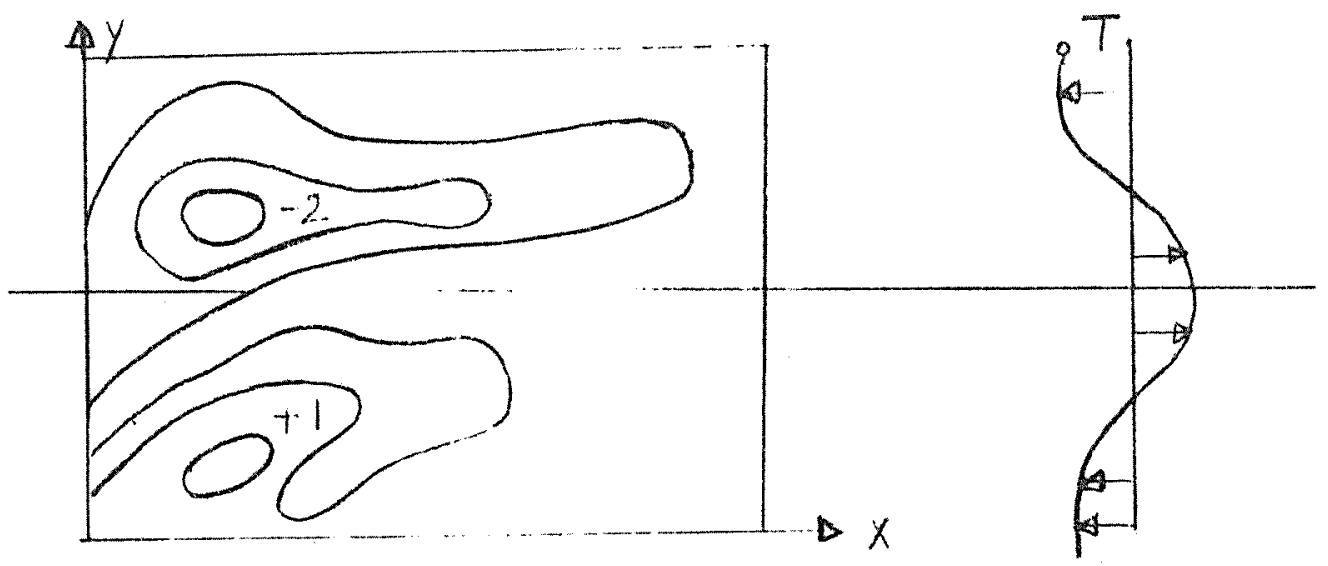

Fig. 4 


\section{Ocean Circulation - Two-Layer Mode1}

1. The Ocean Density Field.

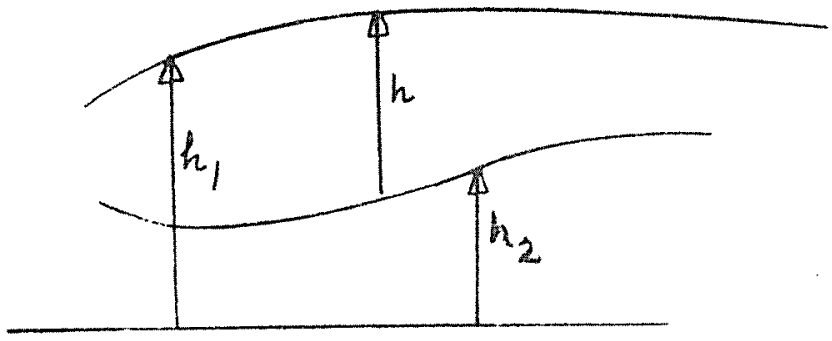

Fig. 1
The main reasons for investigating a twolayer model of the ocean are that the density of the ocean has a distribution. as shown in $\mathrm{F} 1 \mathrm{~g} .2 \mathrm{~A}$,

and that there is a reason to believe that the frictional effects are confined to a thin surface layer. Thus, with a reasonable approximation we can represent the ocean as in Fig. 2B. Furthermore, there is a wind blowing on the surface, causing motion in the upper layer, and for a steady flow with no momentum transfer across the interface, the bottom layer can be taken to be at rest.
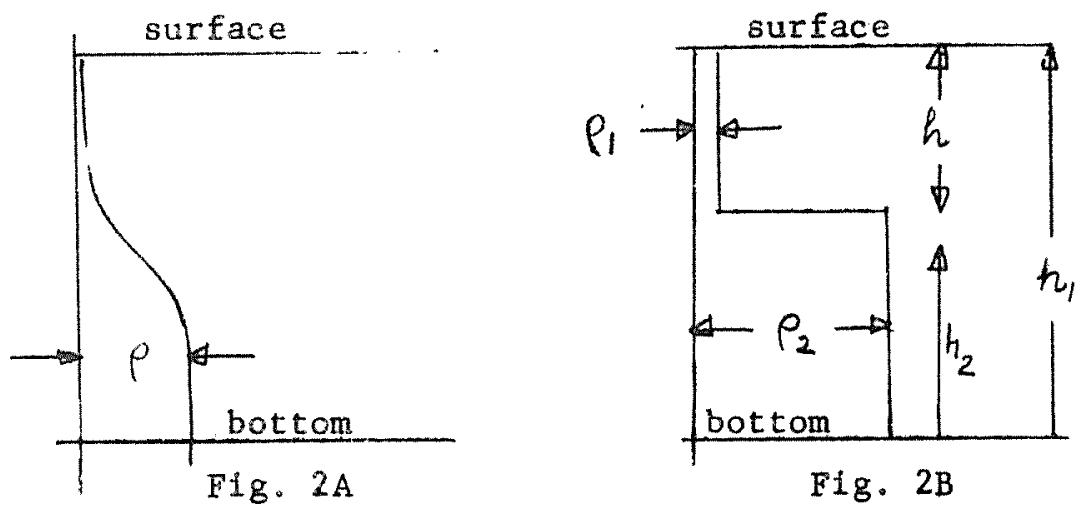

The NavieroStokes equations in a rotating system can now be 
$-110-$

written for a steady state: (with conventional notation)

$$
\begin{gathered}
u u_{1 x}+v u_{1} y-w \mu_{,}-f v=-\frac{1}{p} p_{1 x}+f r(x) \\
u v_{1 x}+v v_{1} y+w v_{, z}+f \mu=-\frac{1}{p} p_{1 y}+f r(x) \\
p_{,} z=-9 p \\
\nabla \cdot \vec{v}=0
\end{gathered}
$$

If we integrate eqn. (95) for both layers, we arrive at:

$$
\begin{gathered}
p_{1}=q p_{1}\left[h_{1}-z\right] \\
p_{2}=q p_{1}\left[h_{1}-h_{2}\right]+g p_{2}\left[h_{2}-z\right]
\end{gathered}
$$

where $z^{2}$ is measured from the bottom.

Taking the gradient of (97) and (98)

$$
\begin{gathered}
\frac{1}{\rho_{1}} \nabla p_{1}=q \nabla h_{1} \\
\frac{1}{\rho_{2}} \nabla p_{2}=\frac{g \rho_{1}}{\rho_{2}} \nabla h_{1}+q \frac{\Delta p}{p_{2}} \nabla h_{2} \\
\text { where } \Delta p=\rho_{1}-\rho_{2} .
\end{gathered}
$$

Since motion in the bottom layer is zero, $\nabla p_{2}=0$ and

$$
\nabla h_{2}=-\frac{\rho_{1}}{\Delta p} h_{1} .
$$

We see that since $\Delta \rho$ is small, even a small change in $h$, will produce large changes in $h_{2}$. Also we can write:

$$
\nabla h=\nabla\left(h_{1}-h_{2}\right)=\frac{\rho_{2}}{\Delta p} \nabla h_{1} .
$$


For the equations of motion of the upper layer the pressure terms become,

$$
\frac{1}{p_{1}} \nabla p_{1}=q^{3} \nabla h
$$

where

$$
q^{\prime}=\frac{\Delta p}{p_{2}} q
$$

which is the well-known reduced-gravity model.

If use is made of the integrated transport concept and the continuity equation is integrated in the vertical, we arrive at,

$$
\frac{\partial}{\partial x} \int_{h_{2}}^{h_{1}} u d z+\frac{\partial}{\partial y} \int_{h_{2}}^{h_{1}} v d z=0
$$

where the boundary terms have canceled out the non-1inear terms. We also can integrate the Navier Stokes equations in the vertical,

$$
\begin{aligned}
& \frac{\partial}{\partial x} \int \mu u d z+\frac{\partial}{\partial y} \int u v d z-f \int v d z=-q^{\prime} h \frac{\partial h}{\partial x}+\int f_{r}(x) d z \\
& \frac{\partial}{\partial x} \int u v d z+\frac{\partial}{\partial x} \int u v d z+f \int u d z=-q^{\prime} h \frac{\partial b}{\partial y}+\int f_{r}(y) d z
\end{aligned}
$$

If the variation of $U, V$, are not great in $z$ direction we make an approximation and write (104), (105) and (106) as the "reduced gravity two-layer" system

$$
\begin{gathered}
\left(h u^{2}\right), x+(h v v), y-f h v=-\frac{q^{\prime}}{2}\left(h^{2}\right), x+f(x) \\
(h u v)_{x}+\left(h v^{2}\right), y+f h u=-\frac{q^{\prime}}{2}\left(h^{2}\right) y+q(y) \\
(h u)_{, x}+(h v)_{, y}=0
\end{gathered}
$$

We will now introduce the following variables and non- 
dimensional groups:

$$
\begin{aligned}
& h_{u}=-\psi_{, y} \\
& h_{v}=\psi, x
\end{aligned}
$$

$$
\begin{gathered}
\psi=\bar{\psi} \psi^{3} ; \mu=\frac{\bar{\psi}}{d b} \hat{\mu} ; v=\frac{\bar{\psi}}{a_{0} d} \hat{v} ; x=a \hat{x} ; y=b \hat{y}, \\
f=f_{0} \hat{f} ; \hat{f}=1+\frac{\beta b}{f_{0}} \hat{y} ; h,=d \hat{h} ; \ell=\frac{a}{b}, \\
r=\frac{q^{3} d^{2}}{2 f_{0} \psi} ; E t=\frac{\bar{\psi}}{f_{0} d a^{2}}
\end{gathered}
$$

where $f_{0}$ is $2 \Omega \times$ sine of the latitude about which we make the expansion.

We rewrite equations 107-109 and dropping caps.,

$$
\begin{gathered}
\ell^{2} \in\left[\left(h u^{2}\right)_{x}+(h \cup v)_{y}\right]-f h v=-\gamma\left(h^{2}\right), x+\frac{q(x) a}{2 f_{0} \bar{y}} \\
\epsilon\left[(h \cup v)_{x}+(h \cup v)_{y}\right]+f h a \cdot-r\left(h^{2}\right)_{, y}+\frac{q(y a}{2 f_{0} \bar{y}} \\
(h u)_{x}+(h v)_{, y}=0
\end{gathered}
$$

2. One-layer model of const. Depth (Fofonoff Inviscid system).

Let us now consider a simple case with the following

as sumptions :

$$
\begin{aligned}
& \text { 1) homogeneous ocean } \\
& \text { 2) no friction } \\
& \text { 3) const. } \frac{h}{\text {. }}
\end{aligned}
$$

The equations of motions for this system are:

$$
\begin{aligned}
& l^{2} \in\left[u u_{1 x}+v u, y\right]-f v=-Z \gamma h, x \\
& \epsilon\left[u v_{x}+v v_{1 y}\right]+f u=-Z \sigma h, y
\end{aligned}
$$


If we differentiate by $-y$ and $x$ respectively and add, we get the vorticity equation,

$$
\underline{V} \cdot \nabla(E S+f)=0
$$

where $\quad \rho=\frac{\partial u}{\partial x}-\ell^{2} \frac{\partial u}{\partial y}$,

and we see that absolute vorticity is conserved along a streamline.

$$
\epsilon S+f=\operatorname{const} \equiv F(\psi) \text {. }
$$

Expanding (115) and saying $\hat{\beta}=\frac{\beta b}{f_{0}}$ (we drop the caps) then:

$$
\epsilon \underline{V} \cdot \nabla \zeta+v \beta=0
$$

Note that in this one layer system

$$
\epsilon=\frac{V_{0}}{f_{0} a}
$$

Typical values for the parameters in $\in$ are

$$
\begin{aligned}
& V_{0}=5 \mathrm{cms} \mathrm{s}^{-1} \\
& f_{0}=10^{-4} \mathrm{p}^{-1} \\
& a=5 \times 10^{8} \mathrm{~cm},
\end{aligned}
$$

which makes

$$
\epsilon \sim 10^{-4}
$$

We now introduce the stream function into equation

$$
\epsilon \nabla^{2} \psi+f-F[\psi]
$$

This model also can be treated by a boundary layer 
analysis. To order $E, V=0$, in the center of the basin, (i.e. no southward velocity),

$$
v=\psi, x=0
$$

and away from the boundaries

$$
\psi=\Psi(y)
$$

Equation (118) becomes

$$
1+\beta y=F(\psi)
$$

\section{Case of const. east-west flow in the basin}

Consider the case where

$$
\Psi \ldots c^{\prime} y
$$

For the interior of the ocean we can write

$$
F(\psi)=1+\frac{\beta}{c} \psi
$$

We substitute into (118)

$$
E \nabla^{2} \psi-\beta / c \psi=-\beta y
$$

Furthermore, let's set $C=1$ for simplicity. We treat the problem now with boundary layers near $x=0$ and $x=1$ in the form,

$$
x=E^{1 / 2} \zeta, 1-x=E^{Y_{\infty}} \zeta \text { respectively. }
$$

Thus, in the boundary layer $\Psi$ and $\beta \psi$ of $1 \mathrm{~g}-$

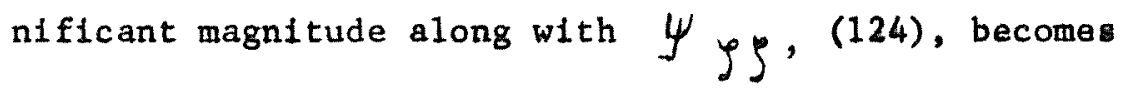

$$
\psi, \zeta_{\zeta}=\beta \psi-\beta y
$$


We can solve for $\psi$ as,

$$
\begin{aligned}
y & =y\left[1-e^{-\sqrt{\beta} \xi}\right] \\
& =y\left[1-e^{-\sqrt{\beta} e^{1 / 2} x}\right]
\end{aligned}
$$

Note that the choice of $\psi=c y$, and $c=1$ makes $\mu: \psi_{X}=-C$ so that there is a westward flow. However if we had chosen $C=-1$ then equation (124) would yield oscillatory solutions and no real boundary layer would result. With the above analysis, we have boundary layers both on the east as well as west side.

To make the flow a closed system, we impose a boundary on the northward side of the basin near $y=1$, and

$$
\begin{aligned}
& 1-y=\epsilon^{1 / 2} \eta \\
& y=1-\ldots \beta \eta
\end{aligned}
$$

gives

on the northern boundary: $\psi-1-e^{-\sqrt{2}} y \epsilon^{1 / 2}$

We can match the solitions and get a flow pattern as In Fig. 3. Fig.4 shows the cut a-a through stream line surface. 


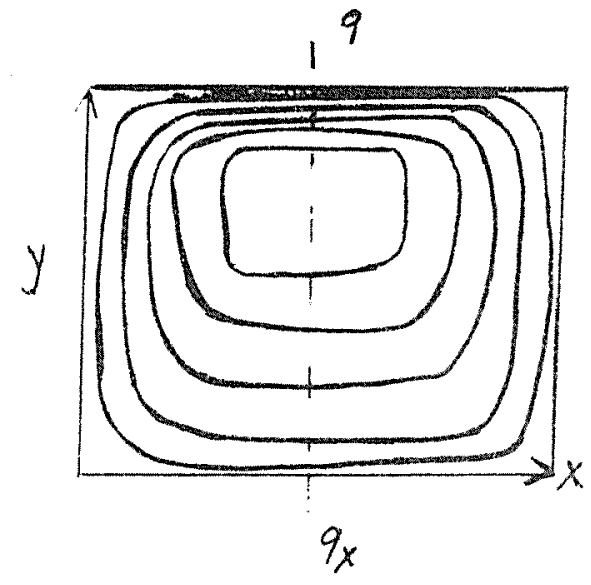

Fig. 3.

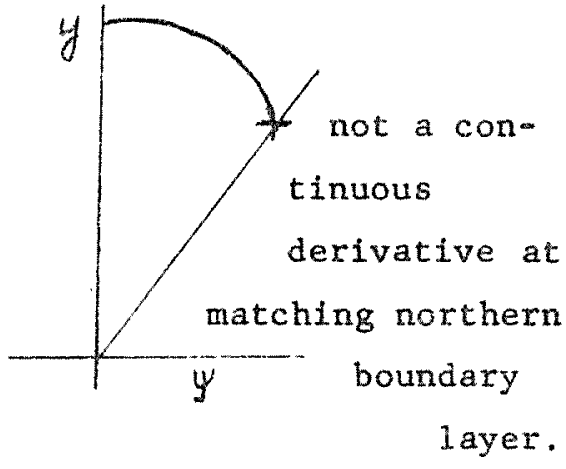

Fig. 4.

It is important to note that Fofonoff's free inertial

solution contains symmetric boundary layers on the eastern and western boundaries and an intense jet across the ocean. This makes a distinct departure from the results of the frictional theories. However, the result is determined by the form of the interior solution which was chosen. If we had admitted a northsouth component of flow in the interfor, the system would have exhibited an east-west asymmetry. 
IX The Inertial Theories of Stommel, Charney and Morgan.

1. Basic Considerations and Formulations.

For the purpose of discussing Charney's and Morgan's paper, we will divide the ocean basin into, three regions (following Morgan). In region II the flow is driven by the wind systems and the solution for the transport is the same as Sverdrup's. In region I the motion is governed by inertial balances, the motion is frictionless, and the non-linear terms are important. In region III the flow is completely unspecified, but we assume that vorticity and energy of the flow entering this area are dissipated by mechanisms which escape our analysis with this model.

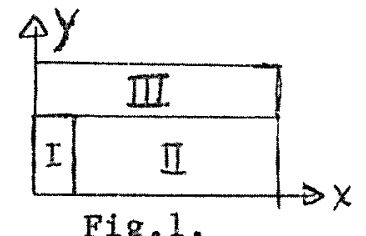

Fig.1.
Charney confines his attention to region $I$, and it is this which we will now formulate with more detail.

The non-dimensional equations of motion for a two-layer ocean (with the same notation as the preceding section), where frictional terms have been omitted are:

$$
\begin{aligned}
l^{2} \in[u \mu, x+v u, y]-f v & =-2 \gamma h, x \\
\epsilon[u v, x+v v, y]+f \mu & =-2 \gamma h, y
\end{aligned}
$$

The continuity equation is

$$
(h u), x+(h v), y=0
$$


The vorticity equation can be constructed from (128), (129), (130) as previously explained in the notes, and

$$
V=\nabla\left(\frac{E S+f}{h}\right)=0
$$

where $\quad S=v_{1, x}-\mathcal{L}^{2} u, y$

The quantity within the parenthesis is the potential vorticity.

The energy or Bernoulli equation corresponding to the above equation can be written as

$$
\nu \circ \nabla\left(\epsilon \frac{\ell^{2} u^{2}+v^{2}}{2}+2 r h\right)=0
$$

It is again convenient to introduce the stream function

$$
\psi_{y}=-h u, \psi_{x}=h v .
$$

In the boundary layer (region I) $\psi, x \gg \Psi_{j y}$, i.e. $v \gg u$.

Then we can approximate equation (132) by

$$
\begin{aligned}
\frac{\widehat{v}^{2}}{2}+2 \tau h & =\text { const. along streamline } \\
& =G(\psi)
\end{aligned}
$$

where $\epsilon U^{3}=\widetilde{v}^{2}$. It is profitable again to "stretch" the $\Varangle$ co-ordinate with

$$
x=\epsilon^{1 / 2} \xi
$$

and we find that

$$
\begin{aligned}
\xi & =v_{1 x} l_{\mu_{y}}^{2}=\epsilon^{-v_{2}} v_{j y}-\ell^{2} \mu_{, y} \\
& \cong \epsilon^{-1} \tilde{v}_{i j}
\end{aligned}
$$

for $\ell^{2} u, y \ll \epsilon^{-1} \tilde{U}_{, y}$. 
Since

$$
v=\frac{1}{h} \psi_{, x}=\frac{1}{h} \varepsilon^{-1 / 2} \psi \cdot \xi
$$

and

$$
\tilde{v}=\frac{1}{2} \psi, \zeta \text {, the energy equation may be written }
$$

as

$$
\frac{\tilde{u}^{2}}{2}+2 \gamma h=\left(\frac{1}{h} \frac{\psi}{2}\right)^{2}+2 \gamma h=G(\psi)
$$

With similar notation and to the same approximations the vorticity equation is,

$$
\frac{\epsilon \cdot \epsilon^{-1} \tilde{v}_{\xi}+f}{h}=\frac{\left(\hbar \psi_{,}\right), g}{h}=F(\psi)
$$

and the $x$-component of the equations of motion reduces to

or

$$
\begin{aligned}
& f v=2 r h x \\
& f \psi \cdot y=\gamma\left(h^{2}\right)_{y}
\end{aligned}
$$

Note this is the geostrophic balance in the north-south direction.

The terms of the y-component of the equation are all of the same order of magnitude, and not influenced by the approximation.

In the interior of the ocean we have very small velocities and relative vorticity so that to a good degree of approximation, the vorticity equation is

$$
\frac{f}{h_{i}}=F\left(\psi_{i}\right)
$$

and the energy equation is

$$
2 \gamma h_{i}=G\left(\psi_{i}\right)
$$




\section{Stomme1's Mode1}

Stommel noted from observational data that:

(The equations are dimenstonal)

$$
\frac{f+V_{i x}}{h} \approx \frac{f}{h_{i}}=\text { const }
$$

Also the equation for geostrophic flow is

$$
f v=q^{\prime} h x
$$

By differentiating the last equation with respect to $X$ and substituting into (139) we obtain

$$
\frac{q^{\prime}}{f} \frac{d^{2} h}{d x^{2}}=\frac{f}{h_{i}} h-f
$$

The solution of (141) with boundary condition that

$$
h=0 \text { at } x=0 \text { is }
$$

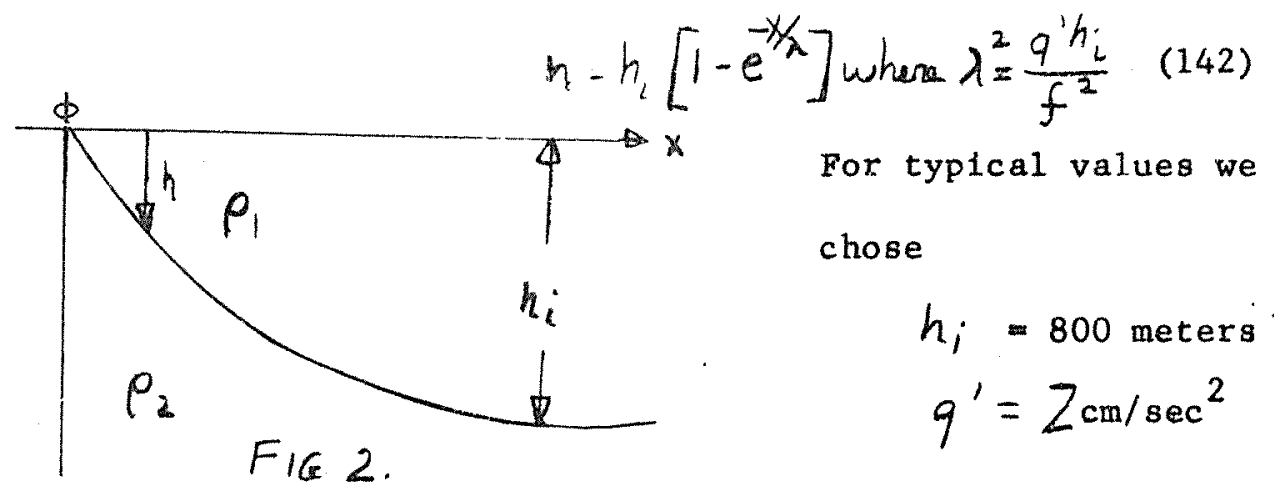

and this simple model gives the mass transport northward in the Gulf Stream as

$$
\begin{aligned}
& \text { as } \int_{0}^{\infty} v h d x=64 \cdot 10^{6} \mathrm{~m}^{3} \mathrm{sec}^{-1} . \\
& v_{\max }=4 \mathrm{~m} \mathrm{~s}^{-1}, \lambda=40 \mathrm{~km} .
\end{aligned}
$$

The mass transport compares favorably with experi- 
mental data, while the width of the siream is perhaps a little too narrow.

3. Morgan's model of the homogeneous ocean.

We now return to the original model, and confine

our attention to a homogeneous ocean, i.e., $h_{i}=1$. In

the interior of the ocean,

$$
F\left(\psi_{i}\right)=f=1+\beta y
$$

Morgan assumed a paraboìic law of wind stress operating in region II,

$$
\tau=\frac{4^{2}}{2}-\frac{1}{8}
$$

We take the curl of the equations of motion for the interior of the ocean

$$
\beta v=-\frac{\partial \tau}{\partial y}=-y
$$

If we introduce the familiar stream function and integrate with condition that $\psi_{i}=0$ at $\quad x=1$, we get:

$$
\psi_{i}=\frac{y}{\beta}[-x+1]
$$

To find the value of $\psi_{1}$ along the boundary between region $I$ and II of the ocean, we may say that $x-0$ in (146) to a very good approximation. Then,

$$
\psi_{i}=\psi=y / \beta \quad \text { at the boundary }
$$

of region $\mathrm{I}$ and $\mathrm{II}$, and 


$$
F(\psi)=1+\beta y=1+\beta^{2} \psi
$$

Since the vorticity is conserved in region I we can substitute (148) into (135) to obtain

$$
\psi, 5,-\beta^{2} \psi 1-f
$$

The solution of (149) leads to the exponential form of streamlines of the type Fofonoff's theory gave.

One should note that since vorticity is introduced in region II and is conserved in region $I$, it must be dissipated in region III in order to have a steady state. Friction must thus play an important role in the ocean, probably in region III, to dissipate this energy.

\section{The Solution According to Charney}

Let us now return to Charney's two-layer model. (A11 equations are dimensional).

The Bernaulli equation is

$$
\frac{v^{2}}{2}+9^{\prime} h=G(\psi)
$$

Integrating across the boundary we obtain

$$
f\left[\psi-y_{i}\right]=\frac{q^{\prime}}{2}\left[h^{2}-h_{i}^{2}\right]
$$

Furthermore,

$$
h v=\Psi_{1}
$$


In the interior of the ocean there is geostrophic

balance and

$$
\mu_{i}=-\frac{1}{h_{i}} \frac{\partial \Psi_{i}}{\partial y}=-\frac{q^{\prime}}{f} \frac{\partial h_{i}}{\partial y}
$$

Charney now uses the observed values of $\psi_{i}$ at the boundary, which can be described by the parabolic law.

$$
\psi_{1}=\psi_{i 0}-c\left(y-y_{0}\right)^{2}
$$

We can also expand $f$ around the latitude where $y=y_{0} \cdot\left(y_{0}\right.$ corresponds to the latitude of Cape Hatteras.)

$$
f=f_{0}+\beta(y-1 \%)
$$

Note the fact that in the $y$ direction, the mass

transport is

$$
\int_{0}^{x} h u d x=\psi_{i}-y_{0}=y_{i}
$$

Thus, Charney chooses

$$
\begin{aligned}
\psi_{i 0} & =80 \times 10^{6} \mathrm{~m}^{3} \mathrm{~s}^{-1} \\
\hat{C} & =2.55 \times 10^{-5} \mathrm{~m} \mathrm{~s}^{-1} \\
f_{0} & =0.84 \times 10^{-4} \mathrm{~s}^{-1} \\
\beta & =1.8 \times 10^{-11} \mathrm{~m}^{-1} \mathrm{~s}^{-1}
\end{aligned}
$$

We can differentiate (154) to obtain

$$
\psi_{i y}=-2 c\left(y-y_{0}\right)
$$

and if we substitute this along with (155) into (153),

$$
\frac{\partial h_{i}^{2}}{\partial y}=-\frac{4 c}{q^{\prime}}\left(y-y_{0}\right)\left[f_{0}+\beta\left(y-y_{0}\right)\right]
$$

which integrated gives 


$$
h_{i}=h_{i 0}^{2}-\frac{2 c}{q^{1}} f_{0}\left(y-y_{0}\right)^{2}-\frac{4 C B}{3 q^{\prime}}\left(y-y_{0}\right)^{3}
$$

and by applying (154)

$$
h_{i}^{2}=h_{i 0}^{2}-\frac{2 f_{0}}{q}\left(\psi_{i 0}-\psi_{i}\right)+\frac{4 C B}{3 q}\left(\frac{\psi_{i 0}-\psi_{i}}{c}\right)^{3 / 2}
$$

At the boundary we have

$$
G\left(\psi_{i}\right)=q^{\prime} h_{i}=q^{\prime}\left[h_{i 0}^{2}-\frac{2 f_{0}}{q^{\prime}}\left(\psi_{i 0}-\psi_{i}\right)+\frac{4 C \beta}{3 q^{2}}\left(\frac{\psi_{i 0}-\psi_{i}}{c}\right)^{3 / 2}\right]^{1 / 2}
$$

Now since $\Psi=\Psi_{i}$ at the boundary and $v=\frac{1}{h_{i}} \frac{d \psi}{d x}$ we may drop the subscript $i$ and obtain, with help of Bernoulli equation, a differential equation for $\psi$ in the region $I$ of the form

$$
\frac{1}{2 h^{2}}\left(\frac{\partial \psi}{\partial x}\right)^{2}=q^{\prime}\left[h_{0}^{2}-\frac{2 f_{0}}{q^{\prime}}\left(\psi_{0}-\psi\right)+\frac{4 c \rho}{3 q^{\prime}}\left(\frac{\psi_{0}-\psi}{c}\right)^{3 / 2}\right]^{1 / 2}
$$

\section{Representation of Charney's Results.}

This equation can be integrated numerically with respect to $X$ to give $\psi$ in the Gulf stream region. Figure $3 \mathrm{~A}$
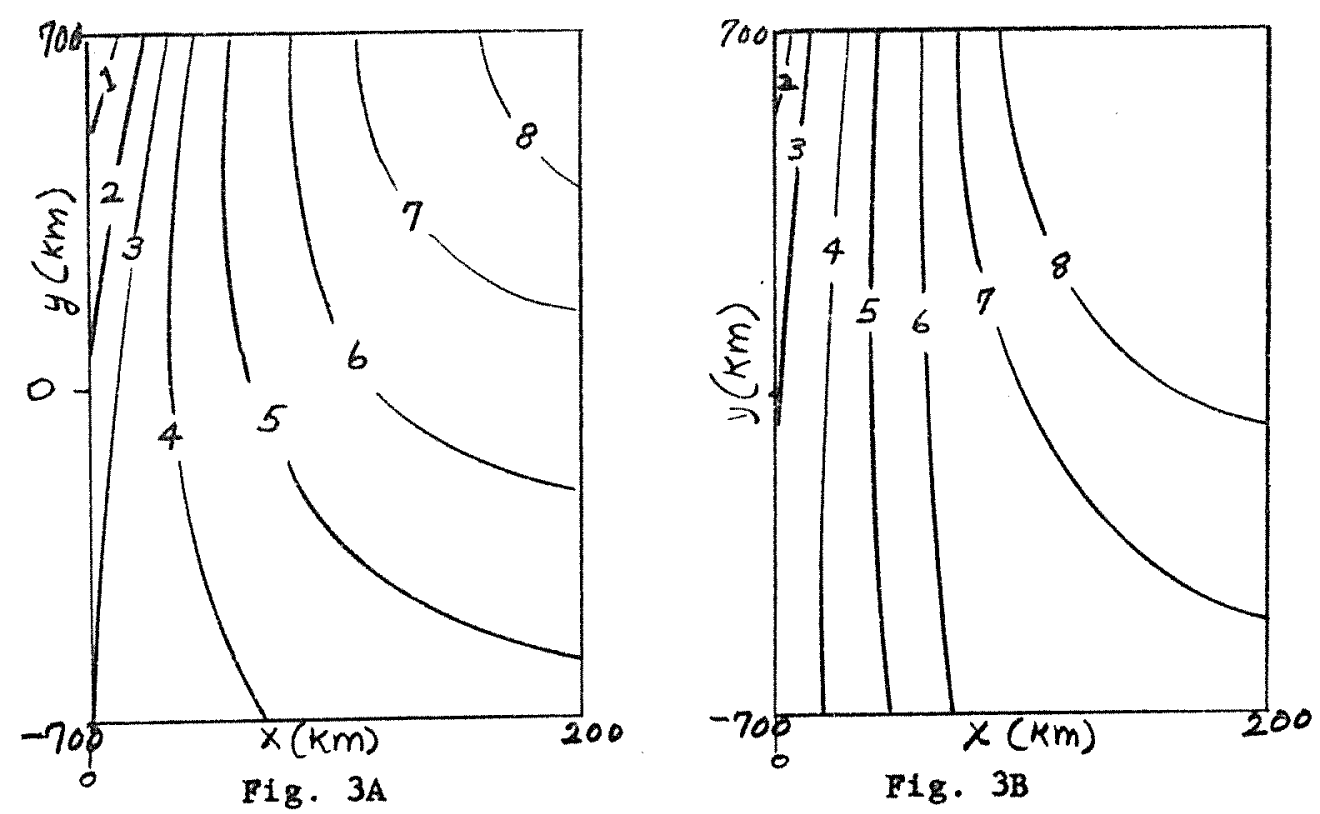
shows Charney's calculated heights of the surface, and Figure 3B shows the observed values. Charney allowed for $d$ Florida Straits transport to enter at the $y=-700$ boundary. Figure 4 shows the velocity as a function of $y$ and $x$.

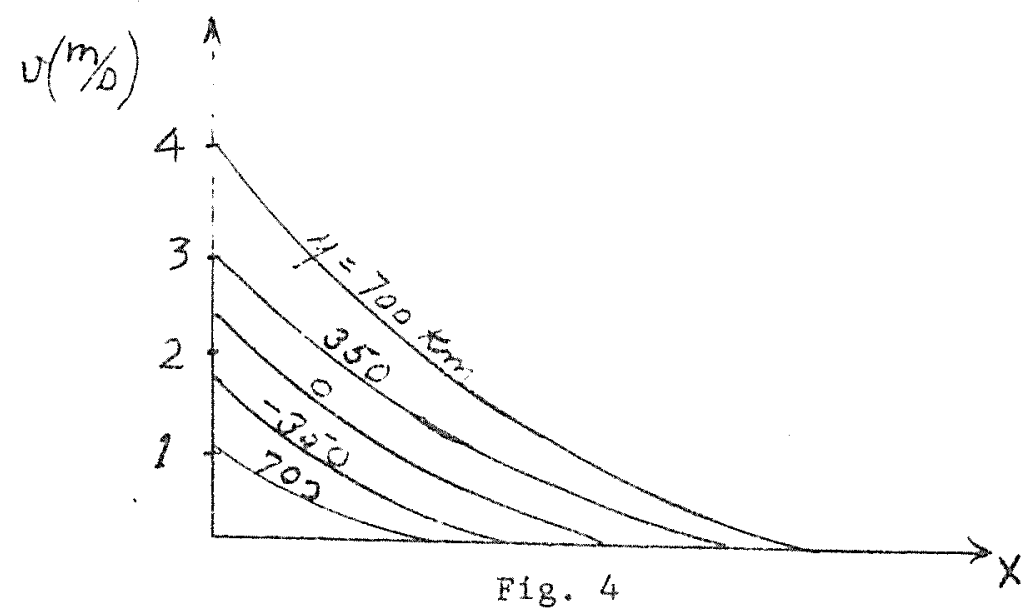

The agreement between the calculated and observed values is rather good, even in view of the approximations made in this theory.

Morgan carried through an analysis for the two-layer system with an analytical form for the wind-stress in the interior basin (II). His results differ quantitatively from Charney's but the analysis and qualitative results are the same. 
X. General Discussion of the Ocean Circulation.

\section{In Retrospect.}

When we consider the theoretical models of the ocean circulation we have described so far, we observe that none of them give a complete and satisfactory description of the actual situation. Munk's linear theory gave a satisfactory description of the Gulf Stream with countercurrent, but his solution contains an unknown parameter, the lateral eddy viscosity $A$, which is chosen so as to make the model yield the actual current width. The question then arises whether there is consistency between the chosen $\mathrm{A}$ and the actual situation. The model with inertially controlled Gulf Stream also gave a fairly good picture of the boundary current. However, it is clear that friction is necessary at some place in the ocean for the vorticity introduced by the wind stresses must be dissipated somewhere. The inertial flow theory does not treat this part of the problem. Morgan suggested that friction is important mostly after the Gulf Stream leaves the coast. Finally it is apparent that the wind-driven circulations discussed thus far give rise to boundary currents only along the western boundary of the ocean.

These considexations led Morgan to look at the circulation patterns allowed in an ocean when the minimum of constraints are imposed on the system. 


\section{The Single-layer system}

In the single layer system we employ the following

constraints:

a) Geostrophy: $\beta v=-\frac{\partial z}{\partial y}$

b) Continuity: $\frac{\partial \mu}{\partial x}=-\frac{\partial v}{\partial y}\left(\frac{1}{\beta} \frac{\partial^{2} \tau}{\partial y^{2}}\right.$ from $\left.a\right)$

c) Conservation of absolute vortictty in the boundary

layer:

$$
J+f=F(\psi)
$$

From a) and b) we see that the field of wind-atreas determines the flow-field in the fnterior. Integrating b) we get

$$
\mu=\frac{x}{\beta} \frac{\partial^{2} T}{\partial y^{2}}+F(y)
$$

By etther choosing $F(y)=0$ or $F(y)=\frac{-b}{\rho} \frac{\partial^{2} z}{\partial y^{2}}$ we can satisfy the boundary condtion $u=0$ at $x$ ar $u=0$ at $x=L$ respectively.

$$
\text { Thus if the interior equations are assumed to hold }
$$
right up to one of the boundaries, $x=0$ or $x=i$, one must add an thertal or viscous boundary layer on the other boundary. Satisfying the boundary condtion $u=0$ on exther $x=0$ or $x=L$ detemines the sign of the eastwest flow in the interior. Morgan looked only at the cases where the boundary current is fed by flow from the interior, but, as pointed out by Veronis, one can also include the cases where the interfor is fed by a boundary current. 
There are then 8 possible flow patterns as indicated below. Of these 4 are ruled out by equation $\mathrm{c}$ ).

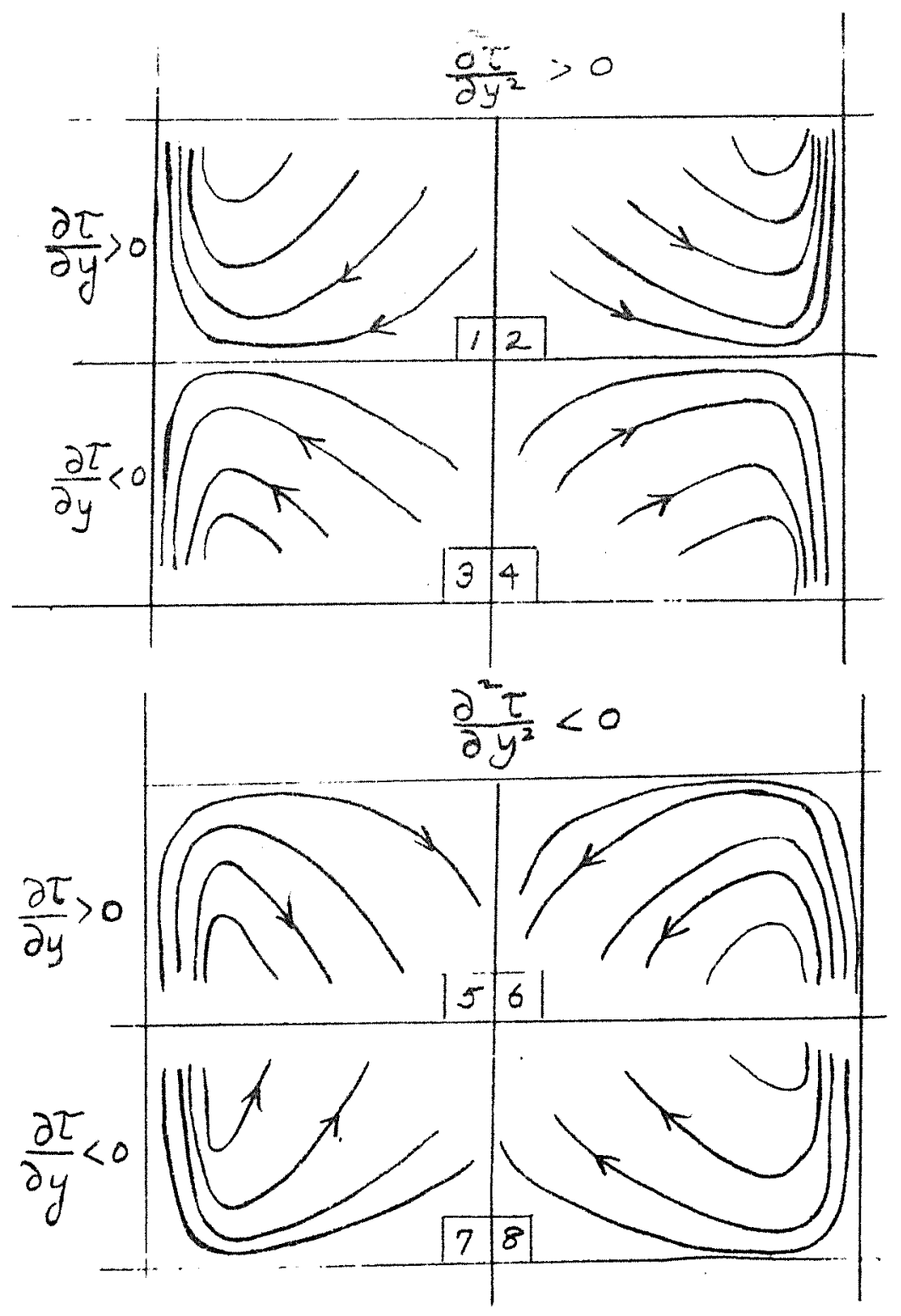

The first 4 cases were treated by Morgan, the last 4 by Veronis. Now it is a simple procedure to rule out the cases $2,4,5$ and 7 as they obviously violate equation c). 
Consider for instance case 1 . Here the relative vorticity in the boundary layer is given by $\frac{\partial v}{\partial x}$ which is negative and decreases in the $y$ direction. The Coriolis parameter in creases in the $y$ direction so it is possible to have $\mathcal{f}+f=$ constant along a streamline.

In case 2 however $\frac{\partial v}{\partial x}$ is positive and increases along the streamline, so does $f$, therefore this case is impossible. A last example: In case $6, \frac{\partial v}{\partial x}$ is positive, but decreases along a streamline therefore since $f$ increases this case is possible. It should be noted that in the cases Morgan treated, only a western boundary current was allowed whereas from the above argument an eastern boundary current is possible as well. A point which must be elaborated in the argunent is that cases 1 and 2 show boundary currents which are fed by the interior solution and consequently the origin of these western boundary currents is fairly clear. In cases 7 and 8 , however, the boundary current formation is not included; indeed, the currents lose their intensity by fanning out into the interior. A possible mechanism for forming these currents will be discussed in connection with the theory of Carrier and Robinson.

\section{The Two-layer System.}

The general conclusions about the possible existence of boundary currents will be drawn from the relationships 
and

$$
\frac{f_{b}+f_{b}}{h_{b}}=\frac{f_{L}}{h_{c}}
$$

$$
\frac{V_{b}^{2}+u_{b}^{2}}{2}+q^{\prime} h_{b}=q^{\prime} h_{i}
$$

where the subscript $i$ refers to the interior of the ocean basin, and the subscript $b$ to the boundary layex. These can be combined to give

$$
\tau_{b}=f_{i}-f_{b}-\frac{v_{0} \underset{\sim}{\sim}}{2 q^{\prime}} \frac{f_{i}}{h_{i}}
$$

We know that $\frac{\underline{v} \cdot \underline{y}}{2 q^{\prime}} \frac{f_{i}}{h_{i}}$ is always positive, therefore, as we investigate each possible pattern of flow, as was done in the single layer case, the important constraint is the sign of $\left(f_{0}-f_{b}\right)$ and relative magnitude of $\frac{\mathcal{L}_{-} \underline{V}}{2 q} \frac{f_{i}}{h_{i}}$. As an example, let us consider case $I$ of the last section. The flow in this case is south-westward and as the flow approaches the western boundary, the stream must be turned northward. The velocity increases and $f_{L}-f_{b}<0$. The vorticity, therefore, decreases as the flow becomes a boundary layer and proceeds northward. This is consistent with the physics of the system. In a similar manner it is possible to show that all flow patterns allowed in the single layer system are also allowed in the two-layer system. In addition, two more flow patterns are permitted. These are illustrated on Fig. 3 and Fig. 4.

Flow as in Figs. 3 and 4 are very unlikely to occur since it is unlikely that very high velocities will be generated before the stream "turns the corner". These high velocities are necessary since we need the effect of change of $\left(f_{i}-f_{b}\right)$ to be 


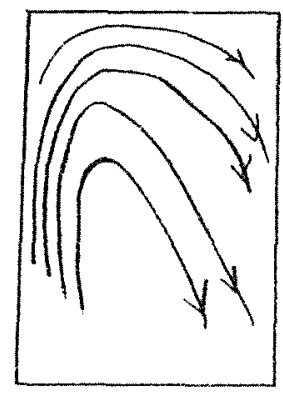

Fig. 3

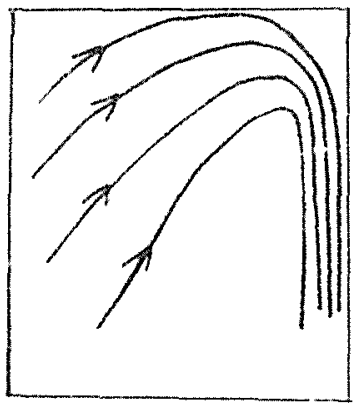

Fig. 4

compensated. In Fig. 3, as the flow proceeds out of the boundary, $f_{i}-f_{b}>0$. To gain vorticity and turn the corner, very high velocities are needed. In Fig. 4, $f_{i}-f_{b}>0$ as the boundary layer starts forming, and to lose vorticity high velocities are needed also.

4. Carrier and Robinson - The Mid-1atitude Jet:

Let us look at an ocean basin and see what flow patterns one can derive from inertial balances only. We must keep in mind that in order to be consistent, frtction must be introduced at some stage, but we will ignore it in this discussion.

In a homogeneous ocean, under geostrophic flow, one chooses

$$
\beta v=-\tau, y=-\sin \pi y
$$

to study the flow which results in a closed basin.

We keep in mind that,

$$
\Psi_{, \varphi}+f=F(\Psi)
$$


is the governing equation in the boundary layer, and away from the boundary

$$
F(\psi)=f
$$

is the governing relation.

$$
\begin{aligned}
& \text { Integrating (163) where } v=+\Psi_{x} \text {, one obtains } \\
& \qquad \psi=\frac{1}{\beta} \sin \pi y[-x+g(y)]
\end{aligned}
$$

Morgan chose

$$
g(y)=1
$$

but Carrier and Robinson pointed out that we may choose $g(y)$ to be a different value in order to match the solutions for a boundary layer also on the eastern boundary. In general, we can say that

$$
g(y)=k
$$

where $K$ can be chosen to match the boundary, layer solution and depends upon the particular latitude of the ocean basin region where we apply the condition. Let us now seek to match the boundary layer on all the four "corners" of the ocean basin.
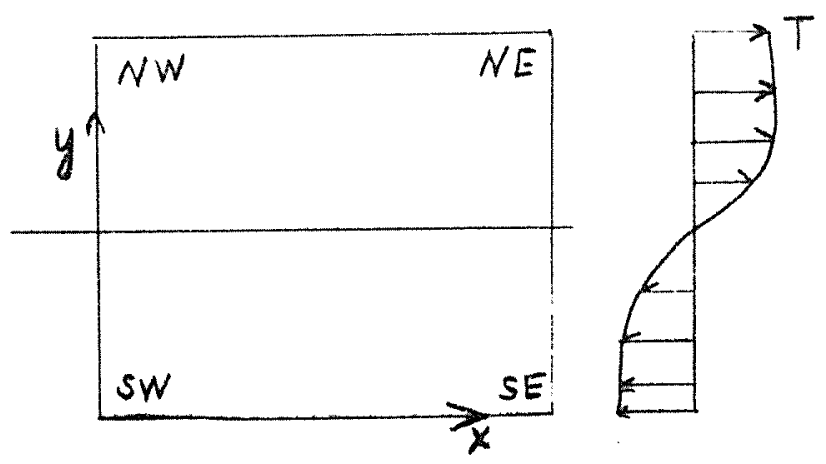

$$
\begin{aligned}
& \text { Near the southern } \\
& \text { boundary of the ocean, } \\
& \sin \pi y \cong \pi y, \\
& \text { and near the northern }
\end{aligned}
$$
boundary,

Fig. 5

$$
\sin \pi y \cong \pi[1-y]
$$

on the south-western edge, $\chi<1$, and one may write 


$$
\begin{aligned}
& -133- \\
& \Psi=\frac{\pi y}{\beta} k^{5}
\end{aligned}
$$

Hence approaching the interior on the south-western part of the basin, we find

$$
F\left(\frac{\pi_{y} k^{s}}{\beta}\right)=1+y
$$

and

$$
F(y)=1+\beta^{2} y / \pi^{k^{s}}
$$

If the boundary current is matched on the southwestern boundary, the governing equation is,

$$
\Psi_{, j 5}-\frac{\beta^{2}}{\pi k^{5}} \psi=-\beta_{y}
$$

Simflarly, on the south-eastern boundary,

$$
\psi_{\zeta \zeta}-\frac{\beta^{2} y}{\pi\left[k^{3}-1\right]}=-\beta_{y}
$$

For non-oscillating solutions of (169) and (170) we

see that

$$
k^{5} \geqslant 0
$$

and

$$
k^{5}-1 \geqslant 0
$$

Therefore, for boundary currents to occur on the boundaries of the southern ocean basin, $K^{s}$ must at least be equal to 1 .

For the northern region

$$
\Psi=\pi\left[\frac{1-y}{\beta}\right]\left[-x+k^{N}\right]
$$

and exactly the same analysis as on the southern boundary yields for the western boundary

$$
k^{N} \leqslant 0
$$


and eastern boundary

$$
k^{N}-1 \leq 0
$$

We, thus, see there is always a boundary current in the northeast if there is a current in the northwest. These are the conclusions of this analysis:

Where $k^{S}=1 \quad\left(k^{N}=0\right)$ in the southern (northern) basin there is only wind-driven transport. If $k^{5}>1\left(R^{N}<0\right)$, the transport is greater than from the wind alone. Therefore, the excess transport is $k^{5}-1$ in the southern basin and $-k^{N}$ in the northern basin.

The method of Carrier and Robinson consists essentially of a superposition of the Fofonoff free solution on the wind-driven interior solution. This is made possible in the non-linear problem by the linearization of the wind-driven transport term. That is, since the inertial balance in the boundary layer is given by $\nabla^{2} \psi+f=F(\psi)$, the linearization of $\sin \pi_{y}$ enables us to linearize $F(\psi)$, and the principle of superposition is applicable to solve this simple equation. We note also that the north-south flow in the interior of the basin is uniquely determined whereas the east-west flow is not. The only restriction on the east-west flow is that $u<0$ everywhere.

It must be recalled that these results are based on the assertion that decaying boundary layers exist in each basin. If this condition is violated, so that oscillatory solutions exist, the results are altered drastically. In particular, it is then possible to have eastward flow in the northern basin, a condition which seems to conform more with observation. 
The circulation which results from the theory of Carrier and Robinson is shown in Fig. 5 where the special case $k^{S}=1, k^{N}=0$ has been chosen. Note that the only way to close the flow is to have a jet across the acean in the vicinity of the latitude of maximum wind-stress curl connecting the southwest

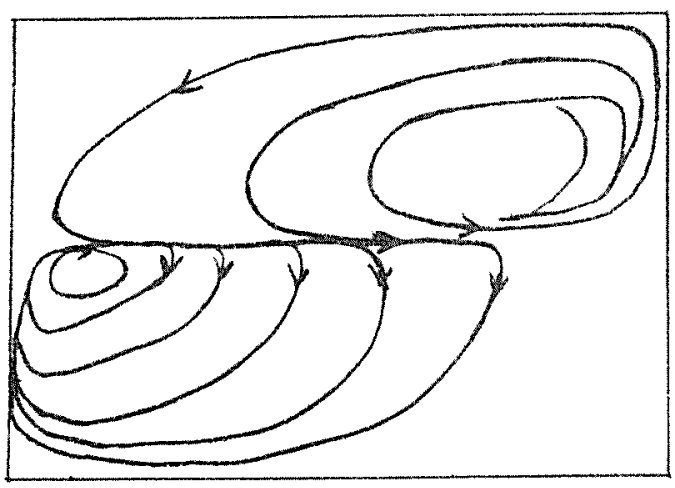

Fig. 5 boundary layer with the northeast boundary layer. Thus the origin of the northeastern boundary layer is dependent on the mid-latitude jet. Also note that if $k^{3}>1$ and $k^{N}<0$, additional boundary layers would have to be included on the northwest and southeast boundaries.

We have not discussed the dynamics of the mid-latitude jet nor the role of friction in the model. It is clear that friction must be included to satisfy the condition of steady state flow (as described by Morgan in connection with the rate of change of vorticity along a streamline).. One can refer to the paper by Carrier and Robinson for a discussion of these phenomena and processes. 


\section{Summary}

The theories to date have yielded a good deal of important information about the large scale features of the wind-driven ocean circulation. Among the many questions which must still be answered we list the following:

a) How much of the "interior" part of the ocean is geostrophically controlled? 1.e., decaying boundary layers indicate that most of the ocean has westward geostrophic flow. Observation indicates large regions of eastward geostrophic flow. Where must one modify the theories with decaying boundary layers to include eastward flow in the interior?

b) Just how does friction come into play in the overall circulation? Is the vorticity dissipated primarily along boundaries or does appreciable dissipation occur in the interior of the ocean?

c) What effect does the density stratification have on the wind-driven circulation?

d) Does the non-1inear interaction between the thermal circulation and the wind-driven circulation invalidate the picture we have formed of the latter? 


\section{References:}

Carrier, G.F. and A.R.Robinson 1961, J.Fl.Mech. (In press). Charney, J.G. 1955. Proc.Nat.Acad.Sci., Hashington, 41, 731. FofonoEf, N.P. 1954. J. Mar.Res., 13, 254.

Morgan, G.W. 1956. Tellus, 8, 301.

Munk, W.H. 1950. J. Meteorology, Z, 79.

P. P. Niller. 